\title{
ZUSES
}

Water Use and Quality of Fresh Surface-Water Resources in the Barataria-Terrebonne Basins, Louisiana

U.S. GEOLOGICAL SURVEY

Open-File Report 98-632

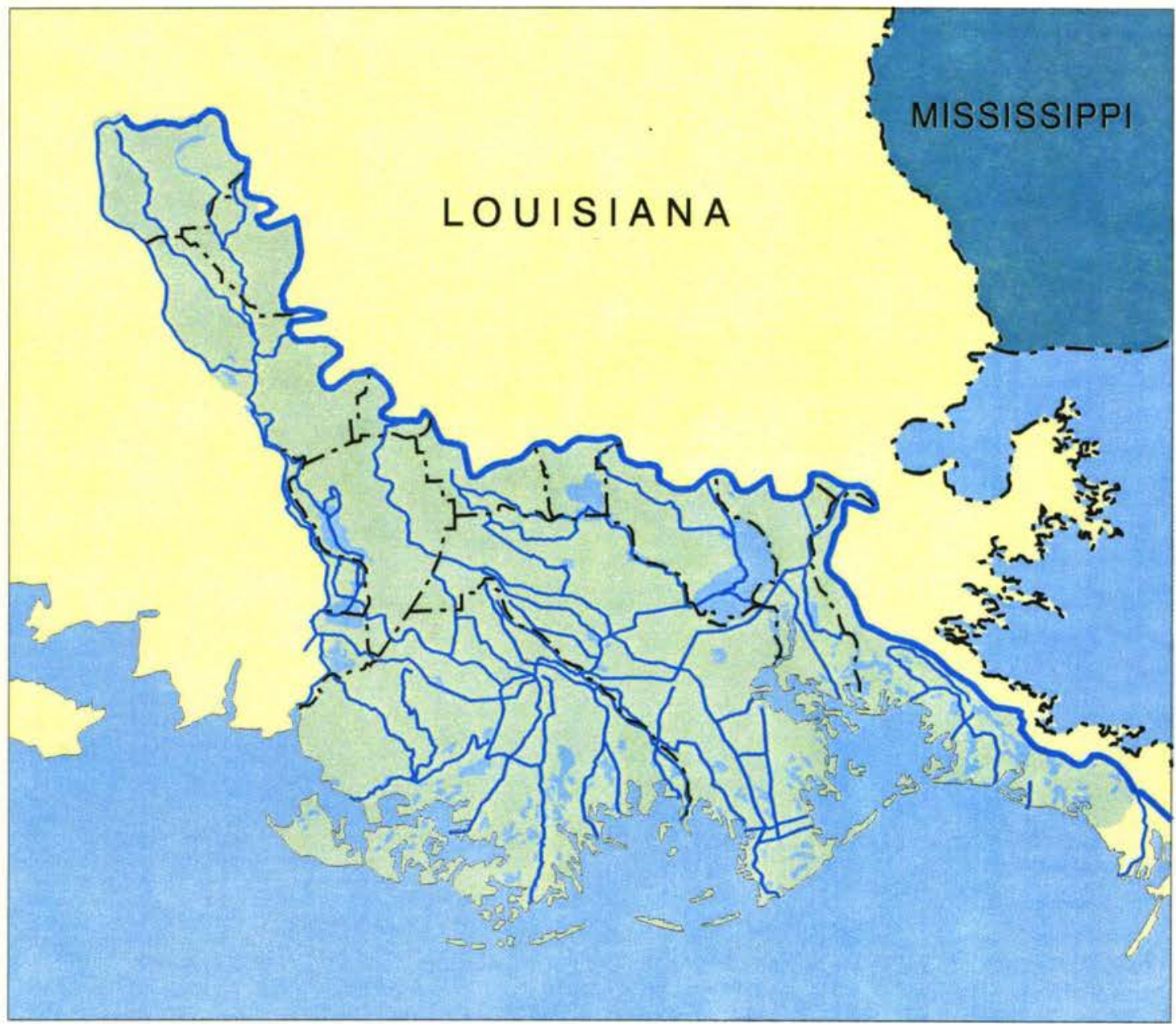

Prepared in cooperation with the

BARATARIA-TERREBONNE NATIONAL

1998

ESTUARY PROGRAM 


\section{WATER USE AND QUALITY OF FRESH SURFACE-WATER RESOURCES IN THE BARATARIA-TERREBONNE BASINS, LOUISIANA}

By Penny M. Johnson-Thibaut, Dennis K. Demcheck, Christopher M. Swarzenski, and Paul A. Ensminger

\section{U.S. GEOLOGICAL SURVEY}

Open-File Report 98-632

Prepared in cooperation with the

BARATARIA-TERREBONNE NATIONAL ESTUARY PROGRAM

Baton Rouge, Louisiana 


\title{
U.S. DEPARTMENT OF THE INTERIOR \\ BRUCE BABBITT, SECRETARY
}

\author{
U.S. GEOLOGICAL SURVEY
}

Charles G. Groat, Director

Any use of trade, product, or firm names is for descriptive purposes only and does not imply endorsement by the U.S. Government.

For additional information contact:

District Chief

U.S. Geological Survey

3535 S. Sherwood Forest Blvd., Suite 120

Baton Rouge, LA 70816

E-mail: dc_la @usgs.gov

Telephone: (225) 389-0281

Fax: (225) 389-0706
Copies of this report can be purchased from:

U.S. Geological Survey

Branch of Information Services

Box 25286

Denver, CO 80225-0286 


\section{CONTENTS}

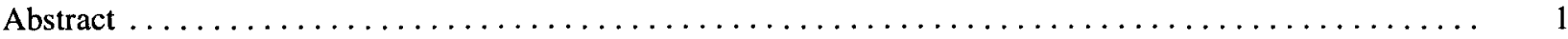

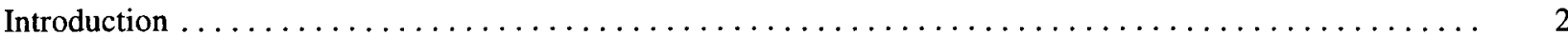

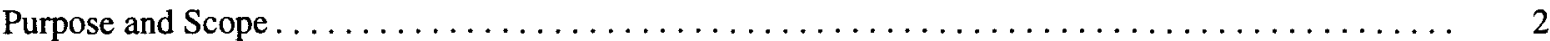

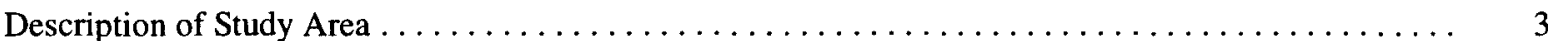

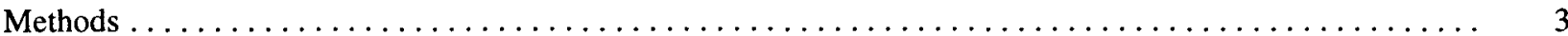

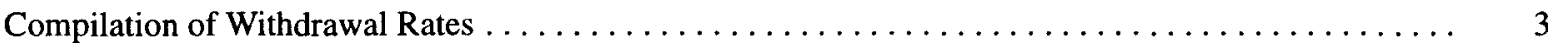

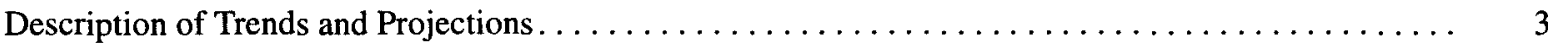

Description of Water Quality $\ldots \ldots \ldots \ldots \ldots \ldots \ldots \ldots \ldots \ldots \ldots \ldots \ldots \ldots \ldots \ldots \ldots \ldots \ldots \ldots \ldots$

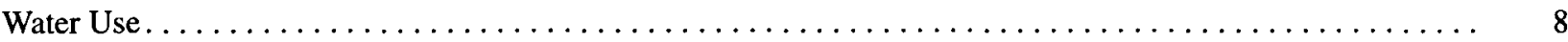

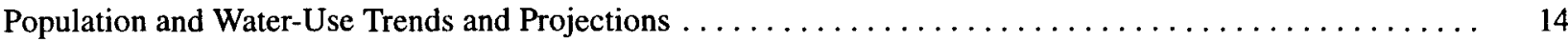

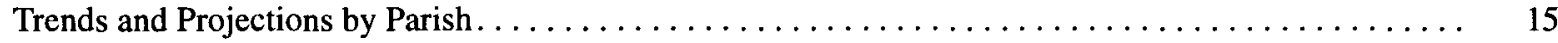

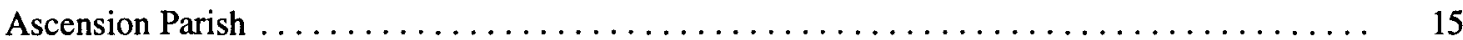

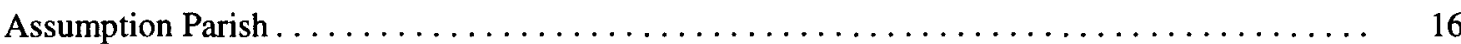

Iberville Parish . . . . . . . . . . . .

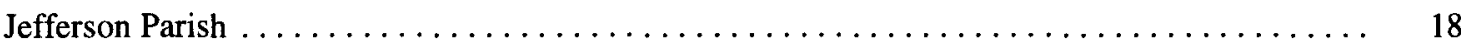

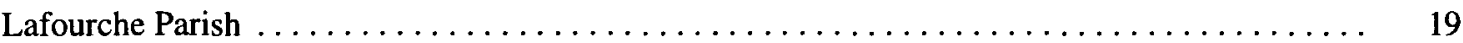

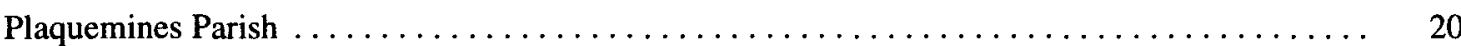

Pointe Coupee Parish ......................................... 21

St. Charles Parish . . . . . . . . . . . . . . . . . . . . . . . . . . . . . . . . . 22

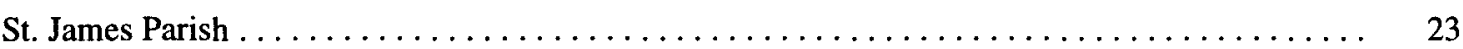

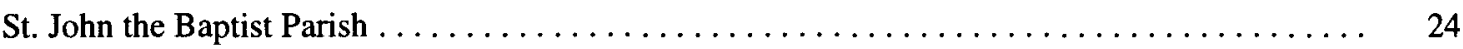

St. Mary Parish. . . . . . . . . . .

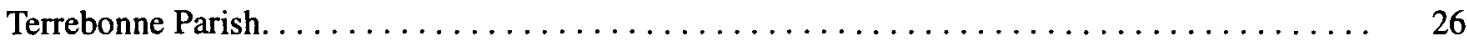

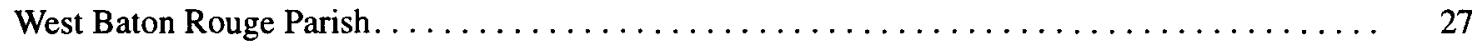

Trend and Projection for Barataria-Terrebonne Basins $\ldots \ldots \ldots \ldots \ldots \ldots \ldots \ldots \ldots \ldots \ldots \ldots \ldots \ldots \ldots$

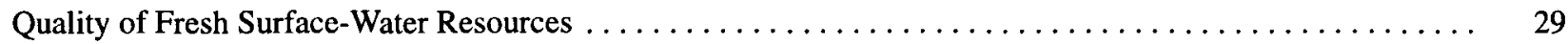

Potential Water-Quality Problems Concerning Diversion of Water from the Mississippi River. . . . . . 30

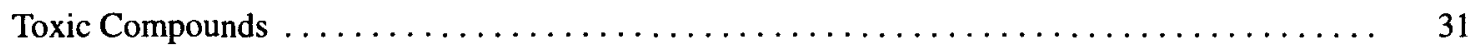

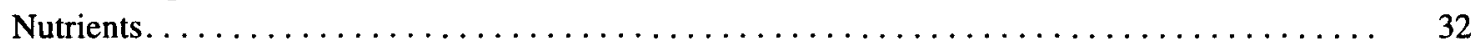

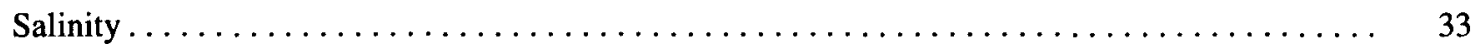

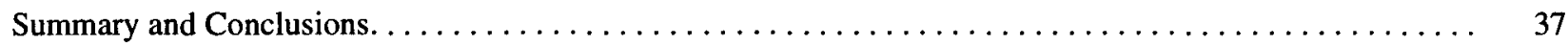

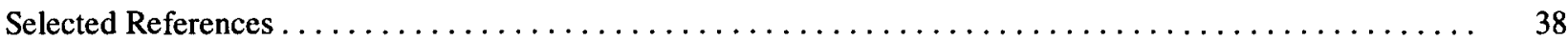

Appendix A: Aggregate ground- and surface-water withdrawal rates and usage in the Barataria-Terrebonne

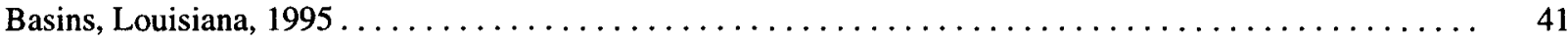

Appendix B: Surface-water withdrawal sites along the Mississippi River adjacent to the Barataria-Terrebonne

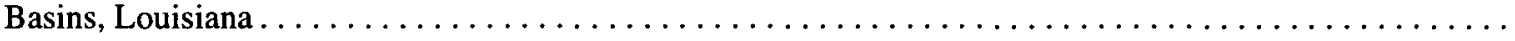

PLATE

1. Map showing location of the study area and surface-water withdrawal sites by selected categories of use in Barataria-Terrebonne Basins and along the Mississippi River, Louisiana, 1995 . . . . . . In pocket

\section{FIGURES}

1-3. Graphs showing historical and projected population and water-withdrawal rates, 1960-2020, for:

1. Ascension Parish within the Barataria-Terrebonne Basins, Louisiana.

2. Assumption Parish within the Barataria-Terrebonne Basins, Louisiana . . . . . . . . . . . 16

3. Iberville Parish within the Barataria-Terrebonne Basins, Louisiana $\ldots \ldots \ldots \ldots \ldots \ldots \ldots \ldots$ 
4-7. Graphs showing historical and projected:

4. Water-withdrawal rates, 1960-2020, for Jefferson Parish within the Barataria-Terrebonne Basins, Louisiana. . . . . . . . . . . . . . . . . . . . . . . . . . . . . . . . . . . . . .

5. Population and water-withdrawal rates, $1960-2020$, for Lafourche Parish within the Barataria-Terrebonne Basins, Louisiana. . . . . . . . . . . . . . . . . . . . . . . . .

6. Water withdrawal rates, $1960-2020$, for Plaquemines Parish within the BaratariaTerrebonne Basins, Louisiana . . . . . . . . . . . . . . . . . . . . . . . .

7. Population and water-withdrawal rates, 1960-2020, for Pointe Coupee Parish within the Barataria-Terrebonne Basins, Louisiana. . . . . . . . . . . . . . . . . . . . . .

8-10. Graphs showing historical and projected water-withdrawal rates, 1960-2020, for:

8. St. Charles Parish within the Barataria-Terrebonne Basins, Louisiana $\ldots \ldots \ldots \ldots \ldots \ldots \ldots .22$

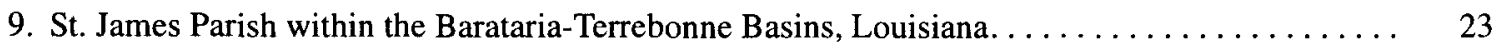

10. St. John the Baptist Parish within the Barataria-Terrebonne Basins, Louisiana. ............ 24

12-13. Graphs showing historical and projected population and water-withdrawal rates, 1960-2020, for:

11. St. Mary Parish within the Barataria-Terrebonne Basins, Louisiana ................ 25

12. Terrebonne Parish within the Barataria-Terrebonne Basins, Louisiana . . . . . . . . . . . 26

13. West Baton Rouge Parish within the Barataria-Terrebonne Basins, Louisiana . . . . . . . . . 27

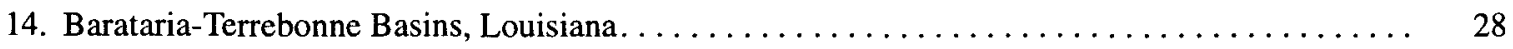

15. Map showing Bayou Lafourche area, Louisiana $\ldots \ldots \ldots \ldots \ldots \ldots \ldots \ldots \ldots \ldots \ldots \ldots \ldots \ldots \ldots \ldots \ldots$

16-19. Graphs showing:

16. Atrazine concentration in the Mississippi River at Baton Rouge, Louisiana, 1991-95 . . . . . .

17. Dissolved nitrate concentration in the Mississippi River at Baton Rouge, Louisiana,

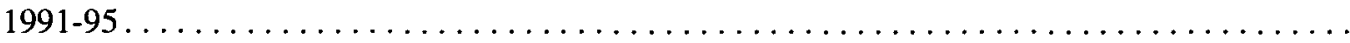

18. Dissolved nitrite plus nitrate concentration, Bayou Lafourche, Louisiana, June 27 and

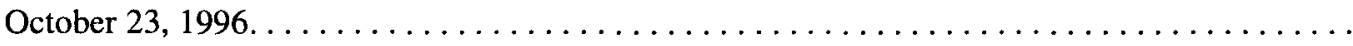

19. Graphs showing A, mean monthly salinity, and B, maximum monthly salinity at selected sites

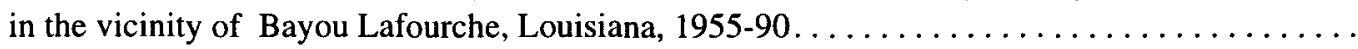

TABLES

1. Regression equation or mean withdrawal rate used to project future ground- and surface-water withdrawal rates for each parish within the Barataria-Terrebonne Basins, Louisiana . . . . . . . . . .

2. The 95-percent confidence intervals for ground- and surface-water withdrawal rate projections for each parish within the Barataria-Terrebonne Basins, Louisiana. . . . . . . . . . . . . . . . .

3. Ground- and surface-water withdrawal sites by selected categories of use, Barataria-Terrebonne Basins, Louisiana, 1995.

4. Water-withdrawal rates for the five largest surface-water public suppliers in the Barataria-Terrebonne

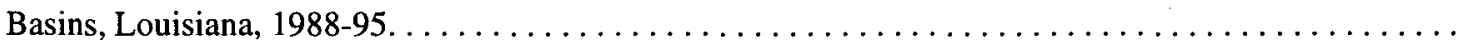

5. Ground- and surface-water withdrawal rates and population in the Barataria-Terrebonne Basins, in Louisiana, 1960-95, and projected ground- and surface-water withdrawal rates and population

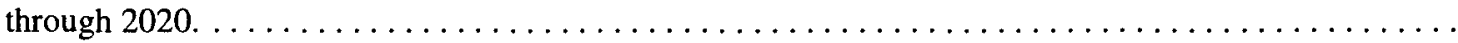

6. Daily mean and maximum salinity, and trends in magnitude for three sites in the Bayou Lafourche-

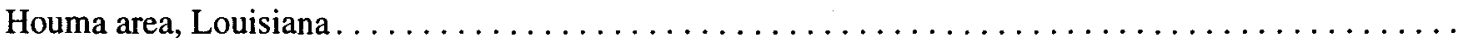

7. Months of lowest and highest salinity for three sites in the Bayou Lafourche-Houma area, Louisiana ... 35

8. Numbers of days per year exceeding chloride concentrations of 250 milligrams per liter at three sites in the Bayou Lafourche area, Louisiana . . . . . . . . . . . . . . . . . . . . . . . 


\title{
WATER USE AND QUALITY OF FRESH SURFACE- WATER RESOURCES IN THE BARATARIA- TERREBONNE BASINS, LOUISIANA
}

\author{
By Penny M. Johnson-Thibaut, Dennis K. Demcheck, Christopher M. Swarzenski, \\ and Paul A. Ensminger
}

\begin{abstract}
Approximately $170 \mathrm{Mgal} / \mathrm{d}$ (million gallons per day) of ground- and surface-water was withdrawn from the Barataria-Terrebonne Basins in 1995. Of this amount, surface water accounted for 64 percent $(110 \mathrm{Mgal} / \mathrm{d})$ of the total withdrawal rates in the basins. The largest surface-water withdrawal rates were from Bayou Lafourche (40 Mgal/d), Bayou Boeuf (14 Mgal/d), and the Gulf Intracoastal Waterway (4.2 Mgal/d). The largest ground-water withdrawal rates were from the Mississippi River alluvial aquifer (29 Mgal/d), the Gonzales-New Orleans aquifer ( $9.5 \mathrm{Mgal} / \mathrm{d}$ ), and the Norco aquifer (3.6 Mgal/d).

The amounts of water withdrawn in the basins in 1995 differed by category of use. Public water suppliers within the basins withdrew $41 \mathrm{Mgal} / \mathrm{d}$ of water. The five largest public water suppliers in the basins withdrew $30 \mathrm{Mgal} / \mathrm{d}$ of surface water: Terrebonne Waterworks District 1 withdrew the largest amount, almost $15 \mathrm{Mgal} / \mathrm{d}$. Industrial facilities withdrew $88 \mathrm{Mgal} / \mathrm{d}$, fossil-fuel plants withdrew $4.7 \mathrm{Mgal} / \mathrm{d}$, and commercial facilities withdrew $0.67 \mathrm{Mgal} / \mathrm{d}$. Aggregate water-withdrawal rates, compiled by parish for aquaculture (37 Mgal/d), livestock $(0.56 \mathrm{Mgal} / \mathrm{d})$, rural domestic $(0.44 \mathrm{Mgal} / \mathrm{d})$, and irrigation uses $(0.54 \mathrm{Mgal} / \mathrm{d})$, totaled about $38 \mathrm{Mgal} / \mathrm{d}$ in the basins. Ninety-five percent of aquaculture withdrawal rates, primarily for crawfish and alligator farming, were from surface-water sources.

Total water-withdrawal rates increased 221 percent from 1960-95. Surface-water withdrawal rates have increased by 310 percent, and ground-water withdrawal rates have increased by 133 percent. The projection for the total water-withdrawal rates in 2020 is $220 \mathrm{Mgal} / \mathrm{d}$, an increase of 30 percent from 1995. Surface-water withdrawal rates would account for 59 percent of the total, or $130 \mathrm{Mgal} / \mathrm{d}$. Surface-water withdrawal rates are projected to increase by 20 percent from 1995 to 2020 .

Analysis of water-quality data from the Mississippi River indicates that the main threats to surfacewater resources are from the herbicide atrazine and excessive nutrients. Atrazine concentrations in the Mississippi River at Baton Rouge briefly exceed the U.S. Environmental Protection Agency maximum contaminant level of 3.0 micrograms per liter during periods in the late spring and early summer. Trace metals in bottom material collected from Bayou Lafourche indicate that the reach of Bayou Lafourche from Donaldsonville to Golden Meadow is adversely affected by low-level contamination. Dissolved nitrate had a mean concentration of 1.4 milligrams per liter in the Mississippi River near Bayou Lafourche and can contribute to excessive plant growth.
\end{abstract}


Long-term salinity records near Bayou Lafourche indicate no pronounced trends, with the exception of the Gulf Intracoastal Waterway at Houma. At this site, salinities remained low until 1961, when the Gulf Intracoastal Waterway was connected to the Gulf of Mexico by the Houma Navigation Canal. The sources of saltwater are variable. Some saltwater has entered Bayou Lafourche south of the Gulf Intracoastal Waterway; at other times saltwater has moved up the Houma Navigation Canal and has flowed east in the Gulf Intracoastal Waterway, north into Company Canal, and southeast in Bayou Lafourche towards Larose, Louisiana.

\section{INTRODUCTION}

In the Barataria-Terrebonne Basins, Bayou Lafourche is the principal source of drinking water for 250,000 people, as well as 50 percent of the water supply for the offshore oil and gas exploration and production industry in the central Gulf of Mexico. All of Lafourche and Assumption Parishes, most of Terrebonne Parish, and parts of Jefferson Parish, get their potable water from Bayou Lafourche (Kirk Cheramie, Bayou Lafourche Freshwater District, written commun., 1997). The Bayou Lafourche Freshwater District (BLFWD) was created in 1950 to provide freshwater to the communities along Bayou Lafourche. Information on water use is essential for planners and managers to resolve problems involving resource allocations, environmental effects, economic development, and water quality. The identification of potential threats to the quality of fresh surface-water resources, especially in areas of extensive water use, is also important. In December 1995, the U.S. Geological Survey (USGS), in cooperation with the Barataria-Terrebonne National Estuary Program (BTNEP), began a 6-month study to evaluate water use and the quality of fresh surface-water resources in the Barataria and Terrebonne Basins, including an analysis of potential threats to the quality of fresh surface-water resources.

The BTNEP was created in 1990 as a partnership between the U.S. Environmental Protection Agency (USEPA) and the State of Louisiana. The BTNEP program's goal is to protect the Barataria-Terrebonne estuary from further degradation and to undertake programs that would begin to check human-induced or natural damage that already had occurred to habitats throughout the estuary (Moore and Rivers, 1995). BTNEP was established under the authority of Section 320 of the Clean Water Act.

\section{Purpose and Scope}

This report describes ground- and surface-water use and the quality of fresh surface-water resources in the Barataria-Terrebonne Basins. This report also addresses projected water-use demands for the years $2000,2005,2010,2015$, and 2020; and threats to the quality of fresh surface-water resources in the basins.

Aggregate ground- and surface-water withdrawal rates from 1960-95 are presented in bar graphs, with projected future water demand through 2020 . Ground- and surface-water withdrawal points for public supply, industrial, commercial, and power generation facilities within the Barataria-Terrebonne Basins in 1995 are tabulated, and surface-water withdrawal points are located on a map. Aggregate water-withdrawal rates in 1995 for irrigation, aquaculture, domestic, and livestock categories are presented in appendix A, and surface-water withdrawal sites along the Mississippi River are listed in appendix B. The discussion on water-quality-related threats to fresh surface-water resources includes potential contamination by toxic compounds, excessive nutrients, and salinity. 


\section{Description of Study Area}

The study area consists of Barataria-Terrebonne Basins in southern Louisiana (pl. 1). The basins, which include more than 4.1 million acres, are bounded by the Mississippi River on the east and the Atchafalaya River on the west. The study area narrows toward the north to the town of Morganza and widens toward the south to the Gulf of Mexico. The basins include all of Assumption, Lafourche, and Terrebonne Parishes and parts of Ascension, Iberville, Jefferson, Plaquemines, Pointe Coupee, St. Charles, St. James, St. John the Baptist, St. Mary, and West Baton Rouge Parishes.

Major streams and canals draining the basins are generally distributaries of the Mississippi River. The Gulf Intracoastal Waterway is a major inland navigation channel that runs east-west across the basins; roughly paralleling the coastline.

The wetlands and open water which comprise almost 80 percent of the basins' area are home to shrimp, oysters, blue crab, and over 60 species of fish. Oil and gas exploration in the basin has created a vast network of canals throughout the basins (Moore and Rivers, 1995). These canals and numerous major navigation channels have increased the potential for inland movement of saltwater into freshwater habitats.

\section{METHODS}

\section{Compilation of Withdrawal Rates}

Data on the ground- and surface-water withdrawal rates were compiled from two sources. Waterwithdrawal data were compiled from the following published reports: Snider and Forbes (1961); Bieber and Forbes (1966); Dial (1970); Cardwell and Walter (1979); Walter (1982); Lurry (1987); Lovelace (1991); and Lovelace and Johnson (1996). The USGS, in cooperation with the Louisiana Department of Transportation and Development (DOTD), has compiled information on water withdrawals and usage on a 5-year interval since 1960. The data are stored in the Louisiana Site-Specific Water Use Data System, a part of the USGS National Site-Specific Water Use Data System (SWUDS). To facilitate the timely completion of each 5-year report, data from the current year and previous year are used. For example, data that were collected for the 1995 water-use report include a composite of water-use data from both 1994 and 1995. Table 4 (presented later in report) includes data taken from quarterly reports with data on a yearly basis from 1988-95. Data included in this table were taken from quarterly reports starting in $1988-89$, when several large users in the State were contacted and asked to report withdrawal rates on a quarterly basis.

\section{Description of Trends and Projections}

Historical ground- and surface-water withdrawal rates were compiled and estimated from USGS paper files and the above mentioned published reports. The first task was to develop a data set that could be used in a regression analysis to project total water-withdrawal rates to the years 2000, 2005, 2010, 2015, and 2020. Site-specific and aggregate data from SWUDS were used to determine ground- and surface-water withdrawal rates for parishes and parts of parishes within the study area for the years 1985, 1990, and 1995. For the period 1960-80, three methods (described below) were used to compile and estimate ground- and surface-water withdrawal rates (determined separately throughout the procedure) that could be used in the regression analysis. These methods were developed through discussions with Susan Hutson (U.S. Geological Survey, oral and written communs., 1998). 
(1) For Assumption, Lafourche, and Terrebonne Parishes, which lie completely within the study area, ground- and surface-water withdrawal rates for the period 1960-80 (every 5 years) were compiled from published water-use reports. For the other 10 parishes, which lie partially within the study area, ground- and surface-water withdrawal rates were estimated for the period 1960-80 as discussed below.

(2) Site-specific withdrawal data that could be traced back to 1960, using USGS paper files, were desirable for estimating the withdrawal rates that were used in the regression analysis. Five of the parishes that lie partially within the study area (Ascension, Iberville, Pointe Coupee, St. Mary and West Baton Rouge) had site-specific public-supply withdrawal rates available. For those five parishes, site-specific public-supply withdrawal rates ( $p s$ area $w u_{60-80}$ ) were totaled for each parish for the area that lies within the study area, for the years 1960-80. Those amounts were then subtracted from the total ground- and surface-water withdrawal rates ( $t o t w u_{60-80}$ ) in the parish for the years $1960-80$ to obtain an adjusted total withdrawal rate (tot adj $w u_{60}$ $80)$.

$$
\text { tot } w u_{60-80}-p s \text { area } w u_{60-80}=\text { tot adj } w u_{60-80}
$$

Ground- and surface-water withdrawal rates (tot area $w u_{85-95}$ ) were totaled for each parish for the areas that lie within the study area for $1985-95$ (every 5 years). Public-supply withdrawal rates within the study area ( $p s$ area $w u_{85-95}$ ) were then subtracted from the total withdrawal rates (tot area $w u_{85-95}$ ) to obtain a rate for each parish (tot area adj $w u_{85-95}$ ) for the years 1985-95.

$$
\text { tot area } w u_{85-95}-p s \text { area } w u_{85-95}=\text { tot area adj } w u_{85-95}
$$

These totals (tot area adj $w u_{85-95}$ ) were then divided by the parish totals for ground- and surface-water withdrawal rates (tot $\left.w u_{85-95}\right)$ for each of the three years $(1985,1990$, and 1995) to determine the percentages of total withdrawal rates that occur within the study area $\left(p c t_{85-95}\right)$. The three percentages were then averaged (av pct ${ }_{85-95)}$.

$$
\left[\left(\text { tot area adj } w u_{85-95} / \text { tot } w u_{85-95}\right)=\text { pct }_{85-95}\right] / 3=a v p c t_{85-95}
$$

The average ( $a v p c_{85-95}$ ) was then multiplied by the adjusted total (tot adj $w u_{60-80}$ ) ground- and surface-water withdrawal rates for each parish for 1960-80 to obtain new adjusted totals (tot area adj $_{60-80}$ ) representative of the area within the study area, excluding site-specific public-supply withdrawal rates for the area. The total site-specific public-supply withdrawal rates ( $p s$ area $w u_{60-80}$ ) within the study area were then added back to the new adjusted totals (tot area $\operatorname{adj}_{60-80}$ ) to obtain withdrawal rates that were representative of the area of the parish within the study area $\left(w u_{60-80}\right)$. These ground- and surface-water withdrawal rates were then used in the regression analysis.

$$
\left(\text { av } \text { pct }_{85-95} * \text { tot adj } w u_{60-80}\right)+\text { ps area } w u_{60-80}=w u_{60-80}
$$

(3) For the five parishes that did not contain any site-specific public-supply withdrawal rates for the part of the parish within the study area (Jefferson, Plaquemines, St. Charles, St. James, and St. John the Baptist), the total site-specific public-supply withdrawal rates for each parish for the years 1960-80 (tot ps wu $60-80$ ) were subtracted from the total withdrawal rates in the parish (tot $w u_{60-80}$ ) to get an adjusted total (tot ${ }_{1}$ adj $w u_{60-80}$ ) for each parish.

$$
\text { tot } w u_{60-80}-\text { tot } p s w u_{60-80}=\operatorname{tot}_{1} \text { adj } w u_{60-80}
$$


Ground- and surface-water withdrawal rates (tot area $w u_{85-95}$ ) were totaled for each parish for the areas that lie within the study area for 1985-95. These totals (tot area $w u_{85-95}$ ) were then divided by the parish totals for ground- and surface-water withdrawal rates $\left(t o t w u_{85-95}\right)$ for each of the three years $(1985$, 1990 , and 1995) to determine the percentages of total withdrawal rates that occur within the study area $\left(\right.$ cct $\left._{85-95}\right)$. The three percentages were then averaged (av pct $\left.{ }_{85-95}\right)$.

$$
\left[\left(\text { tot area } w u_{85-95} / \text { tot } w u_{85-95}\right)=p \text { t }_{85-95}\right] / 3=a v p c t_{85-95}
$$

The average ( $a v$ pct $_{85.95}$ ) was then multiplied by the adjusted total $\left(\right.$ tot $_{l} a d j w u_{60-80}$ ) ground- and surface-water withdrawal rates for each parish for 1960-80 to obtain withdrawal rates that were representative of the area of the parish within the study area $\left(w u_{60-80}\right)$. These ground- and surface-water withdrawal rates were then used in the regression analysis.

$$
\text { av } \text { pct }_{85-95} * \text { tot }_{l} \text { adj } w u_{60-80}=w u_{60-80}
$$

Population data for 1960, 1970, 1980, and 1985 were compiled from the "Missouri State Census Data Center Population Trends Report, 1960-1985," (Urban Information Center, accessed Aug. 1, 1996). Population data for 1965 and 1975 were compiled from USGS files. Population data for 1990-2010 were taken from "Post-Censal Population Projections to 2010 of Louisiana Parishes, 1994," (Louisiana State University Department of Sociology and the Louisiana Population Data Center for the Louisiana Division of Administration, accessed Aug. 1, 1996). Projections of population for 1995 were used instead of the 1995 U.S. Bureau of Census estimates, based on a discussion with the State Demographer, Karen Patterson (oral commun., 1996). Projections of population for 2015 and 2020 were estimated using a ln-ln regression model of population (POPU) against date (YEAR).

$$
\ln (P O P U)=\ln b_{0}+b_{1} \ln (Y E A R)+r_{p}
$$

where,

In is the base-e (natural) logarithm, where $\mathrm{e}=2.7183$;

$b_{0}, b_{1}$ are the regression coefficients and,

$\mathrm{r}_{\mathrm{p}}$ is the residual error equal to $\ln (P O P U)-\ln (P \hat{O P U})$ where $\ln (P \hat{O P U})=\ln b_{0}+b_{1} \ln (Y E A R)$ which can also be expressed as $P \hat{O P U}=b_{0} Y E A R^{b_{1}}$

Standard stepwise regression procedures were performed to project ground- and surface-water withdrawal rates for the period $2000-2020$ using the following multiple regression equation:

$$
W U=d_{0}+d_{1} Y E A R+d_{2} P O P U+r_{w u}
$$


where

WU is either the ground- or surface-water withdrawal rate, and

$\mathrm{d}_{0}, \mathrm{~d}_{1}, \mathrm{~d}_{2}$ are the regression coefficients, and

$\mathrm{r}_{\text {wu }}$ is the residual equal to $W U-\hat{W} U$, where

$\hat{W U}=d_{0}+d_{1} Y E A R+d_{2} P O P U$

The stepwise regression procedure used only explanatory parameters that achieved the significance level of $\alpha=0.15$. Because the withdrawal rates are projected beyond the range of actual data, the results were visually inspected for reasonableness and feasibility. The resulting regression equations are listed in table 1, with 95-percent confidence limits on the 2020 ground- and surface-water withdrawal projections listed in table 2. If no explanatory parameter achieved significance, then the projected rate was assumed to be the mean ground- or surface-water withdrawal calculated for 2000-2020.

Table 1. Regression equation or mean withdrawal rate used to project future ground-and surfacewater withdrawal rates for each parish within the Barataria-Terrebonne Basins, Louisiana

[Mgal/d, in million gallons per day; WU, ground- or surface-water withdrawal rate]

\begin{tabular}{lcc}
\hline \multicolumn{1}{c}{ Parish } & \multicolumn{2}{c}{ Regression equation or mean withdrawal rate (Mgal/d) } \\
\cline { 2 - 3 } & Ground water & Surface water \\
\hline Ascension & WU $=-323+0.165$ (YEAR) & WU $=-0.305+0.000247$ (POPU) \\
Assumption & 6.545 & WU $=-0.584+0.300$ (YEAR) \\
Iberville & 20.342 & 19.457 \\
Jefferson & 9.09500 & 0.150 \\
Lafourche & WU $=-134.715+0.068$ (YEAR) & 36.075 \\
Plaquemines & 0.0 & 1.263 \\
Pointe Coupee & WU $=-537.728+0.275$ (YEAR) & 0.366667 \\
St. Charles & 2.202 & 6.553 \\
St. James & 2.218 & 18.828 \\
St. John the Baptist & 0.227 & 3.645 \\
St. Mary & 0.0 & 4.265 \\
Terrebonne & 10.12 & 15.747 \\
West Baton Rouge & WU $=-548.142+0.281$ (YEAR) & 0.113 \\
\hline
\end{tabular}

${ }^{1}$ Mean withdrawal rate of 1980-95 ground-water values. 
Table 2. The 95-percent confidence intervals for ground-and surface-water withdrawal rate projections for each parish within the Barataria-Terrebonne Basins, Louisiana

[Mgal/d, in million gallons per day; $n / a$, no ground-water withdrawal in that part of the parish within the basins]

\begin{tabular}{lrrrr}
\multicolumn{1}{c}{ Parish } & $\begin{array}{c}\text { Lower bound on the 95-percent } \\
\text { confidence interval for } 2020 \\
\text { (Mgal/d) }\end{array}$ & $\begin{array}{r}\text { Upper bound on the 95-percent } \\
\text { confidence interval for } 2020 \\
\text { (Mgal/d) }\end{array}$ \\
\cline { 2 - 5 } & Ground water & Surface water & Ground water & Surface water \\
\hline Ascension & 3.250 & 2.128 & 17.220 & 3.669 \\
Assumption & -.386 & 9.181 & 13.476 & 36.029 \\
Iberville & .303 & -2.454 & 40.380 & 41.367 \\
Jefferson & 4.028 & -.673 & 14.162 & .973 \\
Lafourche & 1.578 & 23.630 & 4.867 & 48.520 \\
Plaquemines & $\mathrm{n} / \mathrm{a}$ & -.949 & $\mathrm{n} / \mathrm{a}$ & 3.475 \\
Pointe Coupee & .955 & -2.033 & 34.012 & 2.766 \\
St. Charles & -5.341 & -14.250 & 9.745 & 27.356 \\
St. James & .362 & -27.623 & 4.075 & 65.280 \\
St. John the Baptist & .046 & .813 & .419 & 6.477 \\
St. Mary & $\mathrm{n} / \mathrm{a}$ & -9.667 & $\mathrm{n} / \mathrm{a}$ & 18.197 \\
Terrebonne & -.901 & 3.070 & -.280 & 28.424 \\
West Baton Rouge & 6.171 & -.467 & 34.286 & .694 \\
\hline
\end{tabular}




\section{Description of Water Quality}

USGS water-quality data were compiled from the following published reports: Demas and Curwick (1987), Goolsby and others (1995), McGee and Demcheck (1995), Garrison and others (1997), and Wells (1980). Nutrient data from Bayou Lafourche for October 1996 were collected and analyzed by the USGS. The nutrient data were part of the Bayou Lafourche re-evaluation process authorized by the Coastal Wetlands Planning, Protection, and Restoration Act (CWPPRA) and sponsored by the USEPA. All USGS reports and data are available at the USGS office in Baton Rouge, La. Assessments of water bodies in this report as supporting or not supporting designated uses were obtained from Louisiana Department of Environmental Quality publications.

Salinity and chloride data were compiled from various government agencies and public and private sources. The sources utilized included the following organizations: Lafourche Waterworks District 1; U.S. Army Corps of Engineers (COE); Louisiana Department of Health and Hospitals (DHH); City of Lockport Water System; Assumption Waterworks District 1; Nicolaus Paper, Inc.; City of Thibodaux Water System; Louisiana Department of Environmental Quality (DEQ); BTNEP; Terrebonne Waterworks District1; and the USGS.

All water-quality samples collected by the USGS were collected and analyzed according to methods described in Britton and Greeson (1988), Fishman and Friedman (1989), and Shelton (1994). Salinity data were subjected to the nonparametric Kendall's Tau test for trends and magnitude, as described in Wiseman and Swenson (1988). Methods for salinity collection and analysis also are discussed in Reed (1995).

\section{WATER USE}

In 1995, approximately $170 \mathrm{Mgal} / \mathrm{d}$ (million gallons per day) of water were withdrawn from ground- and surface-water sources within the Barataria-Terrebonne Basins. Of this amount, surface water accounted for 64 percent $(110 \mathrm{Mgal} / \mathrm{d})$ of the total withdrawals. The largest surface-water withdrawal rates were $40 \mathrm{Mgal} / \mathrm{d}$ from Bayou Lafourche, $14 \mathrm{Mgal} / \mathrm{d}$ from Bayou Boeuf, and 4.2 Mgal/d from the Gulf Intracoastal Waterway (GIWW). The largest ground-water withdrawal rates were $29 \mathrm{Mgal} / \mathrm{d}$ from the Mississippi River alluvial aquifer, $9.5 \mathrm{Mgal} / \mathrm{d}$ from the Gonzales-New Orleans aquifer, and $3.6 \mathrm{Mgal} / \mathrm{d}$ from the Norco aquifer. Together, these withdrawal rates accounted for 67 percent of the ground water used in the basins. Thirty-eight public water suppliers, 48 industrial facilities, 8 commercial facilities, and 2 fossil-fuel power plants reported withdrawal rates of ground- and surface-water in 1995 (table 3). Of these, 8 public water suppliers, 14 industrial facilities, and 1 commercial facility withdrew only surface water.

Public water suppliers within the basins withdrew $41 \mathrm{Mgal} / \mathrm{d}$ of ground and surface water in 1995 . Of this amount, $25 \mathrm{Mgal} / \mathrm{d}$ were withdrawn from Bayou Lafourche, approximately $4.2 \mathrm{Mgal} / \mathrm{d}$ from the GIWW, $1.0 \mathrm{Mgal} / \mathrm{d}$ from Lower Grand River, and 1.0 Mgal/d from Bayou Boeuf and Bayou Black. Ground-water sources provided the remaining $9.8 \mathrm{Mgal} / \mathrm{d}$ withdrawn for public supply.

The five largest public water suppliers in the basins (table 4) withdrew $30 \mathrm{Mgal} / \mathrm{d}$ of surface water in 1995. Of the five largest public suppliers, Terrebonne Waterworks District 1 withdrew the largest amount in 1995, almost $15 \mathrm{Mgal} / \mathrm{d}$. Industrial facilities withdrew $88 \mathrm{Mgal} / \mathrm{d}$, and fossil-fueled power-generation plants withdrew 4.7 Mgal/d. Aggregate water-withdrawal rates, compiled by parish for aquaculture, livestock, rural domestic and irrigation uses, totaled about $38 \mathrm{Mgal} / \mathrm{d}$ in the basins. Of this amount, withdrawal rates for aquaculture were 37 $\mathrm{Mgal} / \mathrm{d}$; livestock, $0.56 \mathrm{Mgal} / \mathrm{d}$; irrigation, $0.54 \mathrm{Mgal} / \mathrm{d}$; and rural domestic, $0.44 \mathrm{Mgal} / \mathrm{d}$. Ninety-five percent of aquaculture withdrawal rates, primarily for crawfish and alligator farming, were from surface sources. 
Table 3: Ground- and surface-water withdrawal sites by selected categories of use, BaratariaTerrebonne Basins, Louisiana, 1995

[Surface-water withdrawal sites are shown on plate 1, in pocket. Dashes (--) indicate that some withdrawal rates are aggregated under one well or surface-water intake. $\mathrm{Mgal} / \mathrm{d}$, in million gallons per day; WS, public supply; IN, industry; $\mathrm{CO}$, commercial; $\mathrm{PF}$, fossil fuel power]

\begin{tabular}{|c|c|c|c|c|c|c|c|c|}
\hline $\begin{array}{c}\text { Facility } \\
\text { identiff- } \\
\text { cation } \\
\text { number }\end{array}$ & Facility & Use & $\begin{array}{l}\text { Surface-water body } \\
\text { or well number }\end{array}$ & Latitude & Longitude & $\begin{array}{l}\text { Hydrologic } \\
\text { unit code }\end{array}$ & Aquifer ${ }^{1}$ & $\begin{array}{c}\text { With- } \\
\text { drawal } \\
\text { rate } \\
\text { (Mgal/d) }\end{array}$ \\
\hline \multicolumn{9}{|c|}{ ASCENSION PARISH } \\
\hline 357 & People's Water Service & WS & Bayou Lafourche & 300624 & 905931 & 08090301 & & 1.636 \\
\hline \multirow[t]{8}{*}{374} & Industry & $\mathbf{I N}$ & An- 83 & 300658 & 910257 & 08090302 & 112MRVA & -- \\
\hline & & $\mathbf{I N}$ & An- 282 & 300657 & 910256 & 08090302 & 112MRVA & -- \\
\hline & & IN & An- 283 & 300657 & 910256 & 08090302 & 112MRVA & -- \\
\hline & & IN & An- 297 & 300656 & 910249 & 08090302 & 112MRVA & 4.798 \\
\hline & & $\mathbb{I N}$ & An- 298 & 300650 & 910250 & 08090302 & 112MRVA & -- \\
\hline & & IN & An- 380 & 300652 & 910248 & 08090302 & 112MRVA & -- \\
\hline & & $\mathbf{I N}$ & An- 550 & 300653 & 910255 & 08090302 & 112MRVA & -. \\
\hline & & IN & An- 551 & 300653 & 910258 & 08090302 & 112MRVA & -- \\
\hline \multicolumn{9}{|c|}{ ASSUMPTION PARISH } \\
\hline \multirow[t]{3}{*}{381} & Industry & $\mathbf{I N}$ & As- 76 & 295704 & 910241 & 08090301 & 112MRVA & -- \\
\hline & & IN & As- 77 & 295703 & 910243 & 08090302 & 112MRVA & .112 \\
\hline & & $\mathbf{I N}$ & Bayou Lafourche & 295704 & 910132 & 08090302 & & 2.144 \\
\hline 382 & Industry & IN & Bayou Lafourche & 300222 & 910300 & 08090301 & & 2.642 \\
\hline 383 & Industry & IN & Bayou Lafourche & ${ }^{2} 295500$ & ${ }^{2} 905900$ & 08090301 & & 5.162 \\
\hline \multirow[t]{6}{*}{384} & Industry & $\mathbf{I N}$ & As- 72 & 300040 & 910825 & 08090302 & 112MRVA & 5.200 \\
\hline & & $\mathbf{I N}$ & As- 73 & 300037 & 910825 & 08090302 & 112MRVA & -- \\
\hline & & IN & As- 79 & 300026 & 910800 & 08090302 & 112MRVA & -. \\
\hline & & IN & As- 80 & 300050 & 910744 & 08090302 & 112MRVA & -- \\
\hline & & IN & As- 81 & 300046 & 910735 & 08090302 & 112MRVA & -- \\
\hline & & IN & As- 87 & 300049 & 910737 & 08090302 & 112MRVA & -- \\
\hline 385 & Industry & $\mathbf{I N}$ & Lake Verret & 295802 & 910529 & 08090302 & & 3.200 \\
\hline 387 & Assumption Waterworks District 1 & WS & Bayou Lafourche & 295602 & 910102 & 08090301 & & 3.136 \\
\hline \multirow[t]{5}{*}{390} & Industry & IN & As- 15 & 300022 & 910647 & 08090302 & 112 NORC & -- \\
\hline & & IN & As- 16 & 300037 & 910647 & 08090302 & 112NORC & 3.572 \\
\hline & & $\mathbf{I N}$ & As- 60 & 300044 & 910644 & 08090302 & 112NORC & -- \\
\hline & & IN & As- 85 & 300024 & 910630 & 08090302 & 112MRVA & -- \\
\hline & & IN & $\left({ }^{3}\right)$ & 300040 & 910725 & 08090302 & 112MRVA & - \\
\hline \multirow[t]{2}{*}{1723} & Commercial & $\mathrm{CO}$ & $\left({ }^{3}\right)$ & 300050 & 910815 & 08090302 & 112MRVA & .095 \\
\hline & & $\mathrm{CO}$ & $\left({ }^{3}\right)$ & 300045 & 910814 & 08090302 & 112MRVA & $\cdots$ \\
\hline \multicolumn{9}{|c|}{ IBERVILLE PARISH } \\
\hline 509 & Industry & IN & $\left({ }^{3}\right)$ & 302000 & 911445 & 08070300 & I12MRVA & .002 \\
\hline \multirow[t]{2}{*}{577} & Industry & IN & $\left({ }^{3}\right)$ & 301900 & 911415 & 08070300 & 112MRVA & -- \\
\hline & & IN & Cavern Lake & 301831 & 911409 & 08070300 & & 1.104 \\
\hline \multirow[t]{17}{*}{578} & Industry & IN & Ib- 134 & 301856 & 911429 & 08070300 & 112MRVA & -- \\
\hline & & IN & Ib- $180 A$ & 301911 & 911401 & 08070300 & 112SLBR & -- \\
\hline & & $\mathbf{I N}$ & Ib- $180 \mathrm{~B}$ & 301911 & 911401 & 08070300 & 112 SLBR & -. \\
\hline & & $\mathbf{I N}$ & Ib- $180 \mathrm{C}$ & 301911 & 911401 & 08070300 & 112SLBRR & -- \\
\hline & & IN & lb- $180 \mathrm{D}$ & 301911 & 911401 & 08070300 & 112MRVA & -- \\
\hline & & $\mathbf{I N}$ & Ib- 181 & 301857 & 911345 & 08070300 & 112PLQM & -- \\
\hline & & IN & Ib- 182 & 301912 & 911402 & 08070300 & 112PLQM & -- \\
\hline & & $\mathbf{I N}$ & Ib- 183 & 301922 & 911408 & 08070300 & $112 \mathrm{PLQM}$ & .170 \\
\hline & & IN & Ib- 184 & 301905 & 911352 & 08070300 & 112PLQM & -. \\
\hline & & IN & Ib- 242 & 301911 & 911429 & 08070300 & 112MRVA & .057 \\
\hline & & IN & Ib- 260 & 301918 & 911458 & 08070300 & 112MRVA & - \\
\hline & & IN & Ib- 261 & 301914 & 911501 & 08070300 & 112MRVA & -- \\
\hline & & IN & WBR- 151 & 301925 & 911427 & 08070300 & 112MRVA & -- \\
\hline & & $\mathbb{I N}$ & WBR- 163 & 301925 & 911427 & 08070300 & 112MRVA & -- \\
\hline & & IN & WBR- 162 & 301925 & 911449 & 08070300 & 112MRVA & - \\
\hline & & IN & WBR- 142 & 301936 & 911443 & 08070300 & 112MRVA & -. \\
\hline & & $\mathbf{I N}$ & WBR- 157 & 302007 & 911447 & 08070300 & 112MRVA & -- \\
\hline \multirow[t]{3}{*}{580} & Industry & IN & Ib- 62 & 301008 & 910813 & 08090302 & 112MRVA & - \\
\hline & & $\mathbf{I N}$ & Ib- 63 & 301011 & 910803 & 08090302 & 112MRVA & -- \\
\hline & & IN & Ib- 65 & 301036 & 910755 & 08090302 & 112MRVA & -- \\
\hline
\end{tabular}


Table 3: Ground- and surface-water withdrawal sites by selected categories of use, BaratariaTerrebonne Basins, Louisiana, 1995-Continued

\begin{tabular}{|c|c|c|c|c|c|c|c|c|}
\hline $\begin{array}{l}\text { Facility } \\
\text { identifi- } \\
\text { cation } \\
\text { number }\end{array}$ & Facility & Use & $\begin{array}{l}\text { Surface-water body } \\
\text { or well number }\end{array}$ & Latitude & Longitude & $\begin{array}{l}\text { Hydrologic } \\
\text { unit code }\end{array}$ & Aquifer ${ }^{1}$ & $\begin{array}{c}\text { With- } \\
\text { drawal } \\
\text { rate } \\
\text { (Mgal/d) }\end{array}$ \\
\hline \multicolumn{9}{|c|}{ IBERVILLE PARISH--Continued } \\
\hline & & $\mathbf{I N}$ & Ib- 69 & 301009 & 910815 & 08090302 & 112MRVA & - \\
\hline & & IN & Ib- 101 & 301009 & 910815 & 08090302 & 112MRVA & - \\
\hline & & $\mathbf{I N}$ & Ib- 159 & 300756 & 910753 & 08090302 & 112MRVA & -- \\
\hline & & IN & Ib- 269 & 301015 & 910811 & 08070300 & 112MRVA & 4.234 \\
\hline & & $\mathbf{I N}$ & Ib- 343 & 301014 & 910805 & 08090302 & 112MRVA & -- \\
\hline \multirow[t]{5}{*}{584} & Industry & $\mathbb{N}$ & Ib- 227 & 301555 & 911102 & 08070300 & 112DDMRO & -- \\
\hline & & IN & Ib- 228 & 301558 & 911052 & 08070300 & 112DDMRO & -- \\
\hline & & IN & Ib- 244 & 301553 & 911059 & 08070300 & 112MRVA & 3.189 \\
\hline & & IN & Ib- 249 & 301547 & 911105 & 08070300 & 112DDMRO & 1.595 \\
\hline & & IN & Ib- 305 & 301558 & 911101 & 08070300 & 112MRVA & -- \\
\hline 590 & Grosse Tete water system & ws & Ib- $39 \mathrm{~B}$ & 302520 & 912615 & 08070300 & $12220 \mathrm{BR}$ & -- \\
\hline \multirow[t]{2}{*}{591} & Maringouin water system & ws & Ib- 198 & 302926 & 913104 & 08070300 & $12224 \mathrm{BR}$ & 1.176 \\
\hline & & ws & Ib- 29 & 302919 & 913111 & 08070300 & 12220BR & -- \\
\hline \multirow[t]{2}{*}{592} & White Castle water system & ws & lb- 196 & 300959 & 910848 & 08070300 & 112MRVA & -- \\
\hline & & WS & Ib- 223 & 300936 & 910903 & 08070300 & 112MRVA & .127 \\
\hline 595 & Rosedale water system & ws & Ib- 209A & 302629 & 912709 & 08070300 & $12220 \mathrm{BR}$ & .072 \\
\hline \multirow[t]{4}{*}{596} & Iberville Waterworks District 3 & WS & $1 \mathrm{lb}-231$ & 301541 & 910731 & 08070300 & 112DDMRO & -- \\
\hline & & wS & lb- 232 & 301539 & 910728 & 08070300 & 112DDMRO & -- \\
\hline & & ws & Ib- 271 & 301559 & 910805 & 08070300 & 112DDMRO & .000 \\
\hline & & ws & Lower Grand River & 301310 & 911902 & 08070300 & & 1.035 \\
\hline \multirow[t]{2}{*}{994} & Commercial & $\mathrm{CO}$ & Ib- 300 & 301904 & 911840 & 08070300 & 112PLQM & .002 \\
\hline & & $\mathrm{CO}$ & Cavern Lake & 301901 & 911826 & 08070300 & & .130 \\
\hline \multirow[t]{2}{*}{1489} & Myrtle Grove Mobile Home Park & WS & Ib- 276 & 301740 & 911445 & 08070300 & 112MRVA & .012 \\
\hline & & ws & Ib- 275 & 301740 & 911445 & 08070300 & 112MRVA & -- \\
\hline \multirow[t]{2}{*}{1491} & Industry & IN & Ib- 250 & 301542 & 911033 & 08070300 & 112DDMRO & .003 \\
\hline & & IN & Ib-229D & 301542 & 911033 & 08070300 & 112DDMRO & -- \\
\hline 1492 & Iberville Waterworks District 4 & ws & $\left({ }^{3}\right)$ & 301705 & 911415 & 08070300 & 112MRVA & .311 \\
\hline 1494 & Commercial & $\mathrm{CO}$ & $\left(3^{3}\right)$ & 301705 & 911415 & 08070300 & 112MRVA & .000 \\
\hline \multirow[t]{5}{*}{1703} & Industry & $\mathbf{I N}$ & lb- 270 & 301902 & 911330 & 08070300 & 112PLQM & 2.898 \\
\hline & & IN & Ib- 224 & 301900 & 911335 & 08070300 & 112PLQM & - \\
\hline & & IN & Ib- 225 & 301901 & 911333 & 08070300 & 122PLQM & -- \\
\hline & & IN & Ib- 248 & 301900 & 911331 & 08070300 & 122PLQM & - \\
\hline & & IN & Ib- 287 & 301858 & 911335 & 08070300 & 112PLQM & -- \\
\hline 1933 & Industry & $\mathbf{I N}$ & $\left({ }^{3}\right)$ & 301700 & 911400 & 08070300 & 112MRVA & .005 \\
\hline \multicolumn{9}{|c|}{ JEFFERSON PARISH } \\
\hline \multirow[t]{3}{*}{617} & Fossil-fuel power plant & PF & Jf- 25 & 295647 & 900845 & 08090301 & $112 \mathrm{GZNO}$ & -- \\
\hline & & PF & Jf- 28 & 295643 & 900847 & 08090301 & $112 \mathrm{GZNO}$ & -- \\
\hline & & PF & Jf- 43 & 295655 & 900849 & 08090301 & $112 \mathrm{GZNO}$ & 3.400 \\
\hline \multirow[t]{3}{*}{618} & Industry & IN & Jf- 161 & 295549 & 901041 & 08090301 & $112 \mathrm{GZNO}$ & -- \\
\hline & & IN & Jf- 160 & 295518 & 901123 & 08090301 & $112 \mathrm{GZNO}$ & 5.100 \\
\hline & & IN & Jf- 159 & 295536 & 901049 & 08090301 & 112GZNO & -- \\
\hline 619 & Industry & IN & Jf- 151 & 295401 & 900456 & 08090301 & 112GRMC & -- \\
\hline 620 & Industry & IN & Jf- 158 & 295527 & 900839 & 08090301 & $112 \mathrm{GZNO}$ & -- \\
\hline 638 & Industry & IN & Jf- 191 & 295418 & 900741 & 08090301 & $112 \mathrm{GZNO}$ & .804 \\
\hline \multirow[t]{2}{*}{1603} & Industry & $\mathbf{I N}$ & Jf- 132 & 295357 & 900914 & 08090301 & $112 \mathrm{GZNO}$ & .164 \\
\hline & & IN & Jf- 193 & 295356 & 900915 & 08090301 & 112NORC & - \\
\hline \multicolumn{9}{|c|}{ LAFOURCHE PARISH } \\
\hline 189 & Lafourche Waterworks District 1 & ws & Bayou Lafourche & 294656 & 904651 & 08090301 & & 7.939 \\
\hline 190 & Lockport water system & ws & Bayou Lafourche & 293849 & 903216 & 08090301 & & .216 \\
\hline 191 & Thibodaux water system & wS & Bayou Lafourche & 294760 & 904912 & 08090301 & & 3.292 \\
\hline 194 & Industry & IN & Bayou Lafourche & $2_{294800}$ & ${ }^{2} 905100$ & 08090301 & & .081 \\
\hline 195 & Industry & IN & Bayou Lafourche & 2294900 & ${ }^{2} 905300$ & 08090301 & & .025 \\
\hline 239 & ${ }^{4}$ Terrebonne Waterworks District 1 & ws & Bayou Lafourche & 294545 & 904530 & 08090302 & & 8.495 \\
\hline \multirow[t]{2}{*}{982} & Industry & $\mathbf{I N}$ & Lf- 34 & 294357 & 905625 & 08090302 & 112MRVA & -- \\
\hline & & IN & Lf- 35 & 294357 & 905625 & 08090302 & 112MRVA & 1.197 \\
\hline
\end{tabular}


Table 3: Ground- and surface-water withdrawal sites by selected categories of use, BaratariaTerrebonne Basins, Louisiana, 1995-Continued

\begin{tabular}{|c|c|c|c|c|c|c|c|c|}
\hline $\begin{array}{l}\text { Facility } \\
\text { identifi- } \\
\text { cation } \\
\text { number }\end{array}$ & Facility & Use & $\begin{array}{l}\text { Surface-water body } \\
\text { or well number }\end{array}$ & Latitude & Longitude & $\begin{array}{l}\text { Hydrologic } \\
\text { unit code }\end{array}$ & Aquifer ${ }^{1}$ & $\begin{array}{c}\text { With- } \\
\text { drawal } \\
\text { rate } \\
\text { (Mgal/d) }\end{array}$ \\
\hline \multicolumn{9}{|c|}{ LAFOURCHE PARISH--Continued } \\
\hline 1804 & Industry & IN & Bayou Lafourche & 294000 & 903500 & 08090302 & & .338 \\
\hline 1805 & Industry & IN & Bayou Lafourche & 293800 & 902830 & 08090302 & & 2.133 \\
\hline 1993 & Industry & IN & Bayou Lafourche & 294355 & 903521 & 08090301 & & 2.766 \\
\hline \multicolumn{9}{|c|}{ POINTE COUPEE PARISH } \\
\hline \multirow[t]{3}{*}{32} & New Roads water system & Ws & PC- 65 & 304147 & 912522 & 08070300 & $12228 \mathrm{BR}$ & .345 \\
\hline & & ws & PC- 170 & 304155 & 912628 & 08070300 & 12228BR & .575 \\
\hline & & Ws & PC- 321 & 304157 & 912832 & 08070300 & $12203 \mathrm{FP}$ & .575 \\
\hline \multirow[t]{3}{*}{33} & Morganza water system & wS & PC. 69 & 304411 & 913547 & 08070300 & $12228 \mathrm{BR}$ & .066 \\
\hline & & wS & PC- 150 & 304411 & 913549 & 08070300 & 12228BR & .000 \\
\hline & & WS & PC- 301 & 304430 & 913549 & 08070300 & 12112BR & .000 \\
\hline \multirow[t]{7}{*}{34} & Fossil-fuel power plant & PF & PC- 161 & 304023 & 912111 & 08070300 & $12101 \mathrm{FP}$ & -- \\
\hline & & PF & PC- 162 & 304019 & 912121 & 08070300 & $12101 \mathrm{FP}$ & .372 \\
\hline & & PF & PC- 163 & 304018 & 912116 & 08070300 & $12101 \mathrm{FP}$ & .411 \\
\hline & & PF & PC- 180 & 304322 & 912219 & 08070300 & 12112BR & .011 \\
\hline & & $\mathrm{PF}$ & PC- 181 & 304323 & 912218 & 08070300 & $12112 \mathrm{BR}$ & .012 \\
\hline & & $\mathrm{PF}$ & PC- 245 & 304321 & 912219 & 08070300 & $12101 \mathrm{FP}$ & .041 \\
\hline & & PF & PC- 288 & 304025 & 912112 & 08070300 & $12101 \mathrm{FP}$ & .413 \\
\hline \multirow[t]{4}{*}{224} & Fordoche water system & ws & PC- 282 & 303552 & 913759 & 08070300 & 12102FP & .109 \\
\hline & & ws & PC. 151 & 303530 & 913629 & 08070300 & 12117BR & -- \\
\hline & & ws & PC- 57 & 303544 & 913757 & 08070300 & 12112BR & - \\
\hline & & ws & PC- 54 & 303542 & 913650 & 08070300 & $12220 \mathrm{BR}$ & - \\
\hline \multirow[t]{3}{*}{812} & Livonia water system & ws & PC- 138 & 303357 & 913304 & 08070300 & $12220 \mathrm{BR}$ & -- \\
\hline & & ws & PC- 147 & 303404 & 913254 & 08070300 & 12220BR & .101 \\
\hline & & WS & PC- 276 & 303404 & 913254 & 08070300 & $12115 \mathrm{BR}$ & .050 \\
\hline 813 & Lottie Waterworks & ws & PC- 148 & 303315 & 913836 & 08070300 & 12117BR & .020 \\
\hline 814 & Pointe Coupee Waterworks District 2 & ws & PC- 292B & 304343 & 913858 & 08070300 & $12102 \mathrm{FP}$ & .039 \\
\hline \multirow[t]{3}{*}{816} & Brownview Community water system & ws & PC- 196 & 304316 & 913457 & 08070300 & 12228BR & .043 \\
\hline & & ws & PC- 189 & 304320 & 913452 & 08070300 & 12228BR & -- \\
\hline & & ws & PC- 194 & 304305 & 913402 & 08070300 & $12228 \mathrm{BR}$ & -. \\
\hline \multirow[t]{7}{*}{817} & Industry & IN & PC- 17 & 303538 & 912328 & 08070300 & 12117BR & .088 \\
\hline & & IN & PC- 19 & 303538 & 912326 & 08070300 & 112MRVA & .913 \\
\hline & & IN & PC- 21 & 303537 & 912328 & 08070300 & $11206 \mathrm{BR}$ & .913 \\
\hline & & IN & PC- 22 & 303538 & 912326 & 08070300 & $11206 \mathrm{BR}$ & -- \\
\hline & & IN & PC- 154 & 303538 & 912325 & 08070300 & $12117 \mathrm{BR}$ & .135 \\
\hline & & IN & PC- 291 & 303542 & 912327 & 08070300 & $11206 \mathrm{BR}$ & .428 \\
\hline & & IN & PC- 319 & 303537 & 912323 & 08070300 & 112MRVA & .917 \\
\hline 990 & Industry & IN & PC- 224 & 303206 & 912510 & 08070300 & 12220BR & 1.015 \\
\hline \multirow[t]{4}{*}{997} & Pointe Coupee Water District 1 & ws & PC- 268 & 303802 & 912100 & 08070300 & 12115BR & .126 \\
\hline & & ws & PC- 267 & 303827 & 912845 & 08070300 & $12203 \mathrm{FP}$ & .086 \\
\hline & & ws & PC- 176 & 303840 & 912000 & 08070300 & 12220BR & .000 \\
\hline & & ws & PC- 215 & 303640 & 912434 & 08070300 & $12224 \mathrm{BR}$ & .000 \\
\hline \multirow[t]{3}{*}{1535} & False River Waterworks Corp. & ws & PC- 203 & 303926 & 912910 & 08070300 & 12228BR & .198 \\
\hline & & ws & PC- 157 & 303927 & 912912 & 08070300 & $12228 \mathrm{BR}$ & -- \\
\hline & & wS & PC- 152 & 303600 & 912418 & 08070300 & $12224 \mathrm{BR}$ & .097 \\
\hline \multirow[t]{6}{*}{1536} & M \& S water system & WS & PC- 257 & 304056 & 912449 & 08070300 & 12228BR &.- \\
\hline & & WS & PC- 131 & 304031 & 912727 & 08070300 & $12203 \mathrm{FP}$ & .053 \\
\hline & & ws & PC- 122 & 304052 & 912518 & 08070300 & 12203FP & -- \\
\hline & & WS & PC- 112 & 303637 & 912419 & 08070300 & $12102 \mathrm{FP}$ & .027 \\
\hline & & WS & PC- 59 & 304036 & 912155 & 08070300 & 12224BR & .027 \\
\hline & & ws & PC- 262 & 304047 & 912658 & 08070300 & 12228BR & 0.053 \\
\hline 1543 & $\begin{array}{l}\text { Pointe Coupee Waterworks } \\
\text { Corporation }\end{array}$ & WS & PC- 211 & 304339 & 912557 & 08070300 & 12228BR & .077 \\
\hline 1656 & John Lefeaux water system & ws & PC- 159 & 303138 & 913205 & 08070300 & 12220BR & .025 \\
\hline 1772 & Commercial & $\mathrm{CO}$ & $\left({ }^{3}\right)$ & 303155 & 913148 & 08070300 & 12220BR & .007 \\
\hline 1834 & Commercial & $\mathrm{Co}$ & $\left({ }^{3}\right)$ & 304344 & 912920 & 08070300 & 12228BR & .004 \\
\hline 1835 & Frisco water well & WS & PC. 204 & 303505 & 913142 & 08070300 & 12220BR & .003 \\
\hline 1987 & Torbert-Frisco water system & WS & PC- 153 & 303425 & 913008 & 08070300 & 12220BR & .050 \\
\hline 1989 & Pointe Coupee Area Water & WS & $\left({ }^{3}\right)$ & 304237 & 912546 & 08070300 & $12228 \mathrm{BR}$ & .029 \\
\hline
\end{tabular}


Table 3: Ground-and surface-water withdrawal sites by selected categories of use, BaratariaTerrebonne Basins, Louisiana, 1995-Continued

\begin{tabular}{|c|c|c|c|c|c|c|c|c|}
\hline $\begin{array}{l}\text { Facility } \\
\text { identifi- } \\
\text { cation } \\
\text { number }\end{array}$ & Facility & Use & $\begin{array}{l}\text { Surface-water body } \\
\text { or well number }\end{array}$ & Latitude & Longitude & $\begin{array}{l}\text { Hydrologic } \\
\text { unit code }\end{array}$ & Aquifer ${ }^{1}$ & $\begin{array}{c}\text { With- } \\
\text { drawal } \\
\text { rate } \\
\text { (Mgal/d) }\end{array}$ \\
\hline \multicolumn{9}{|c|}{ POINTE COUPEE PARISH--Continued } \\
\hline 2006 & E. Lejeune water system & ws & $\left({ }^{3}\right)$ & 303456 & 913136 & 08070300 & $12117 \mathrm{BR}$ & .012 \\
\hline 2008 & Waterloo Water Service & ws & $\left({ }^{3}\right)$ & 304125 & 912420 & 08070300 & 12228BR & .040 \\
\hline \multicolumn{9}{|c|}{ ST. CHARLES PARISH } \\
\hline 1848 & Industry & IN & SC- 167 & 295849 & 902712 & 08090301 & 112NORC & .003 \\
\hline \multirow[t]{3}{*}{1937} & Industry & IN & Humble Canal & 295124 & 902727 & 08090301 & & 5.900 \\
\hline & & IN & SC- 166 & 295136 & 902712 & 08090301 & 112NORC & .036 \\
\hline & & IN & SC- 165 & 295140 & 902705 & 08090301 & 112 NORC & -- \\
\hline \multicolumn{9}{|c|}{ ST. JAMES PARISH } \\
\hline \multirow[t]{6}{*}{910} & Industry & IN & SJ- 4 & 295938 & 905045 & 08090301 & 112GRMC & -. \\
\hline & & IN & SJ- 5 & 295938 & 905045 & 08090301 & 112GRMC & - \\
\hline & & IN & SJ- 6 & 295940 & 905050 & 08090301 & 112GRMC & - \\
\hline & & IN & SJ- 7 & 295941 & 905045 & 08090301 & $112 \mathrm{GRMC}$ & -- \\
\hline & & IN & SJ- 172 & 295938 & 905030 & 08090301 & 112GRMC & -- \\
\hline & & IN & SJ. 173 & 295938 & 905050 & 08090301 & 112GRMC & 1.091 \\
\hline \multicolumn{9}{|c|}{ ST. JOHN THE BAPTIST PARISH } \\
\hline \multirow[t]{3}{*}{924} & Industry & IN & SJB- 169 & 300214 & 903421 & 08090301 & 112GRMC & -- \\
\hline & & IN & SJB- 171 & 300215 & 903416 & 08090301 & 112GRMC & .172 \\
\hline & & IN & Des Allemands & 300245 & 903400 & 08090301 & & 2.671 \\
\hline \multicolumn{9}{|c|}{ ST. MARY PARISH } \\
\hline 1122 & St. Mary Water District 3 & ws & Bayou Boeuf & 2294100 & ${ }^{2} 910700$ & 08090302 & & .800 \\
\hline \multirow[t]{2}{*}{1128} & Commercial & $\mathrm{CO}$ & Bayou Boeuf & ${ }^{2} 294100$ & 2910600 & 08090302 & & .243 \\
\hline & & $\mathrm{CO}$ & Bayou Caroline & 2294100 & ${ }^{2} 910600$ & 08090302 & & .005 \\
\hline 1564 & Industry & $\mathrm{IN}$ & Bayou Boeuf & 2294200 & ${ }^{2} 910700$ & 08090302 & & 12.839 \\
\hline 1861 & Industry & IN & $\begin{array}{l}\text { Gulf Intracoastal } \\
\text { Waterway }\end{array}$ & ${ }^{2} 294100$ & 2910600 & 08090302 & & .002 \\
\hline \multicolumn{9}{|c|}{ TERREBONNE PARISH } \\
\hline \multirow[t]{2}{*}{239} & ${ }^{4}$ Terrebonne Waterworks District 1 & wS & $\begin{array}{l}\text { Gulf Intracoastal } \\
\text { Waterway }\end{array}$ & 293428 & 904302 & 08090302 & & 4.170 \\
\hline & & ws & Bayou Black & 293431 & 904330 & 08090302 & & .581 \\
\hline 1692 & Industry & IN & $\left({ }^{3}\right)$ & 292600 & 903700 & 08090302 & 112MRVA & .054 \\
\hline 1901 & Industry & IN & Bayou Black & 2293400 & ${ }^{2} 905800$ & 08090302 & & .003 \\
\hline 1902 & Industry & IN & Private Canal & 293740 & 905545 & 08090302 & & .065 \\
\hline 1903 & Industry & IN & $\begin{array}{l}\text { Houma Navigation } \\
\text { Canal }\end{array}$ & ${ }^{2} 292100$ & ${ }^{2} 904400$ & 08090302 & & 2.235 \\
\hline \multicolumn{9}{|c|}{ WEST BATON ROUGE PARISH } \\
\hline \multirow[t]{7}{*}{35} & W. Baton Rouge Gas And Water & ws & WBR- 98 & 302842 & 911226 & 08070300 & 12110BR & -- \\
\hline & & ws & WBR- 132 & 302505 & 911320 & 08070300 & 12115BR & -- \\
\hline & & ws & WBR- 150 & 302827 & 911254 & 08070300 & 12110BR & 0.856 \\
\hline & & ws & WBR- 152 & 302927 & 911302 & 08070300 & 12108BR & .856 \\
\hline & & ws & WBR- 153 & 302959 & 911248 & 08070300 & 12108BR & .000 \\
\hline & & ws & WBR- 154 & 303002 & 911858 & 08070300 & $12112 \mathrm{BR}$ & -- \\
\hline & & ws & WBR- 164 & 303002 & 911858 & 08070300 & 12110BR & -- \\
\hline \multirow[t]{3}{*}{36} & Commercial & $\mathrm{CO}$ & WBR- 36 & 302610 & 911211 & 08070300 & 12112BR & - \\
\hline & & $\mathrm{CO}$ & WBR- 37 & 302613 & 911215 & 08070300 & 12112BR & - \\
\hline & & $\mathrm{CO}$ & WBR- 181 & 302644 & 911212 & 08070300 & 12117BR & .177 \\
\hline \multirow[t]{3}{*}{37} & Plaquemine water system & ws & WBR- III & 302550 & 911241 & 08070300 & 12220BR & .000 \\
\hline & & ws & WBR- 112 & 302550 & 911241 & 08070300 & $12115 \mathrm{BR}$ & .774 \\
\hline & & ws & WBR- 113 & 302547 & 911232 & 08070300 & 12115BR & .774 \\
\hline \multirow[t]{3}{*}{38} & Port Allen water system & ws & WBR- 35 & 302657 & 911242 & 08070300 & 12112BR & .155 \\
\hline & & ws & WBR- 110 & 302733 & 911254 & 08070300 & 12112BR & .372 \\
\hline & & ws & WBR- 4 & 302733 & 911224 & 08070300 & $12117 \mathrm{BR}$ & .105 \\
\hline \multirow[t]{2}{*}{39} & W. Baton Rouge Water District 4 & ws & WBR- 164 & 303002 & 911858 & 08070300 & 12110BR & .046 \\
\hline & & WS & WBR-176 & 303229 & 912146 & 08070300 & $12115 \mathrm{BR}$ & .163 \\
\hline
\end{tabular}


Table 3: Ground-and surface-water withdrawal sites by selected categories of use, BaratariaTerrebonne Basins, Louisiana, 1995-Continued

\begin{tabular}{|c|c|c|c|c|c|c|c|c|}
\hline $\begin{array}{c}\text { Facility } \\
\text { identifi- } \\
\text { cation } \\
\text { number }\end{array}$ & Facility & Use & $\begin{array}{l}\text { Surface-water body } \\
\text { or well number }\end{array}$ & Latitude & Longitude & $\begin{array}{l}\text { Hydrologic } \\
\text { unit code }\end{array}$ & Aquifer $\mathbf{1}^{1}$ & $\begin{array}{c}\text { With. } \\
\text { drawal } \\
\text { rate } \\
(\mathbf{M g a l} / \mathrm{d})\end{array}$ \\
\hline \multicolumn{9}{|c|}{ WEST BATON ROUGE PARISH--Continued } \\
\hline & & WS & WBR- 177 & 303227 & 912414 & 08070300 & 12115BR & .163 \\
\hline \multirow[t]{3}{*}{41} & W. Baton Rouge Water District 2 & WS & WBR- 173 & 302456 & 911302 & 08070300 & $12115 \mathrm{BR}$ & .302 \\
\hline & & WS & WBR- 173 & 302456 & 911302 & 08070300 & 12115BR & .302 \\
\hline & & WS & WBR- 132 & 302505 & 911320 & 08070300 & 12115BR & .287 \\
\hline 415 & Commercial & $\mathrm{CO}$ & WBR- 103 & 302522 & 911227 & 08070300 & 12115BR & .013 \\
\hline \multirow[t]{2}{*}{578} & Industry & IN & WBR- 140 & 301948 & 911424 & 08070300 & 112MRVA & -- \\
\hline & & IN & WBR-141 & 302007 & 911447 & 08070300 & 112MRVA & .775 \\
\hline \multirow[t]{2}{*}{1059} & Industry & IN & WBR- 155 & 302042 & 911515 & 08070300 & 112MRVA & -. \\
\hline & & IN & WBR- 156 & 302038 & 911516 & 08070300 & 112MRVA & .607 \\
\hline \multirow[t]{2}{*}{1060} & Industry & IN & WBR- 171 & 302019 & 911508 & 08070300 & 112MRVA & .. \\
\hline & & IN & WBR- 197 & 302023 & 911508 & 08070300 & 112MRVA & - \\
\hline \multirow[t]{2}{*}{1061} & Industry & IN & WBR- 118 & 301942 & 911643 & 08070300 & 112MRVA & .175 \\
\hline & & IN & WBR- 203 & 301946 & 911644 & 08070300 & 112MRVA & -- \\
\hline \multirow[t]{5}{*}{1062} & Industry & IN & WBR- 24 & 302348 & 911403 & 08070300 & $112 S L B R$ & -- \\
\hline & & IN & WBR- 79 & 302349 & 911400 & 08070300 & 112MRVA & 1.556 \\
\hline & & IN & WBR- 191 & 302343 & 911402 & 08070300 & 112MRVA & -- \\
\hline & & IN & WBR- 192 & 302344 & 911352 & 08070300 & 112MRVA & -- \\
\hline & & IN & WBR- 193 & 302347 & 911358 & 08070300 & 112MRVA & -- \\
\hline \multirow[t]{2}{*}{1065} & Industry & IN & WBR- 167 & 301924 & 911504 & 08070300 & 112MRVA & - \\
\hline & & IN & WBR- 168 & 301928 & 911508 & 08070300 & 112MRVA & .091 \\
\hline \multirow[t]{2}{*}{1066} & W. Baton Rouge Waterworks District 1 & WS & WBR- 126 & 302133 & 911552 & 08070300 & 112MRVA & .. \\
\hline & & WS & WBR- 145 & 302128 & 911556 & 08070300 & 112MRVA & .231 \\
\hline \multirow[t]{2}{*}{1103} & Industry & IN & WBR- 38 & 302836 & 911249 & 08070300 & 12112BR & .072 \\
\hline & & IN & WBR- 44 & 302833 & 911245 & 08070300 & 12112BR & .072 \\
\hline 1473 & Westport Properties & WS & WBR- 137 & 302657 & 911421 & 08070300 & 12112BR & .144 \\
\hline 1982 & Industry & IN & $\left({ }^{3}\right)$ & 302800 & 911300 & 08070300 & 112MRVA & .002 \\
\hline
\end{tabular}

'Aquifer: 112MRVA, Mississippi River alluvial; 112NORC, Norco; 112SLBR, Shallow sands of Baton Rouge area; 112DDMRO, Deltaic deposits of Mississippi River Valley, older; 12220BR, "2,000-foot" sand of Baton Rouge area; 12224BR, "2,400-foot" sand of Baton Rouge area; 112PLQM, Plaquemines; 112GZNO, Gonzales-New Orleans; 12228BR, "2,800-foot" sand of Baton Rouge area; 12203FP, Zone 3 Florida Parishes and Pointe Coupee Parish; $12112 B R$, "1,200-foot" sand of Baton Rouge area; 12101FP, Zone 1 Florida Parishes and Pointe Coupee Parish; 12102FP, Zone 2 Florida Parishes and Pointe Coupee Parish; $12117 B R$ "1,700-foot" sand of Baton Rouge area; 12115BR, "1,500-foot" sand of Baton Rouge area; 11206BR, "600-foot" sand of Baton Rouge area; 112GRMC, Gramercy; $12110 \mathrm{BR}$, "1,000-foot" sand of Baton Rouge area; 12108BR, "800-foot" sand of Baton Rouge area.

2 Estimated latitude and longitude.

${ }^{3}$ Not available.

${ }^{4}$ Terrebonne Waterworks District 1 is listed in Lafourche and Terrebonne Parishes but is counted as one public water supplier. 
Table 4. Water-withdrawal rates for the five largest surface-water public suppliers in the Barataria-Terrebonne Basins, Louisiana, 1988-95

[Withdrawal rates are in million gallons per day. --, no data collected from these public suppliers in 1988]

\begin{tabular}{lcccccccc}
\hline Water supplier & 1988 & 1989 & 1990 & 1991 & 1992 & 1993 & 1994 & 1995 \\
\hline $\begin{array}{l}\text { Terrebonne Water- } \\
\text { works District 1 }\end{array}$ & 14.61 & 16.50 & 15.31 & 13.17 & 12.86 & 13.02 & 13.25 & 14.62 \\
$\begin{array}{l}\text { Lafourche Water- } \\
\text { works District 1 }\end{array}$ & 7.53 & 7.94 & 8.20 & 8.19 & 7.91 & 7.66 & 7.94 & 8.32 \\
$\begin{array}{l}\text { Assumption Water- } \\
\text { works District 1 }\end{array}$ & -- & 2.63 & 2.68 & 2.60 & 3.05 & 2.95 & 3.14 & 3.17 \\
$\begin{array}{l}\text { Thibodaux water } \\
\text { system }\end{array}$ & -- & 2.64 & 3.04 & 2.97 & 2.82 & 2.87 & 3.29 & 2.88 \\
$\begin{array}{l}\text { Iberville Water- } \\
\text { works District 3 }\end{array}$ & -- & 1.04 & .83 & .80 & .80 & .92 & 1.04 & 1.03 \\
\hline
\end{tabular}

POPULATION AND WATER-USE TRENDS AND PROJECTIONS

Population projections were presented for each parish for the period 2000-2020. Water-use projections for each parish were made for the period 2000-2020. The methods used for the projections are described in the "Methods" section. Projections for each of the 13 parishes are discussed, and large increases or decreases in historical water use are explained. 


\section{Trends and Projections by Parish}

\section{Ascension Parish}

About 8.5 percent of Ascension Parish is located within the boundaries of the Barataria-Terrebonne Basins. In 1995, surface-water withdrawal rates for public supply accounted for 25 percent (1.6 Mgal/d) of the total water-withdrawal rates of $6.6 \mathrm{Mgal} / \mathrm{d}$. Total water-withdrawal rates increased 189 percent from 1960-95 (fig. 1). Multiple regression was used for the projected ground- and surface-water withdrawal rates. In 2020, total water-withdrawal rates are projected as $13 \mathrm{Mgal} / \mathrm{d}$ for an increase of 100 percent from 1995. The fluctuation in ground-water withdrawal rates from 1985-95 was due to one industrial facility.

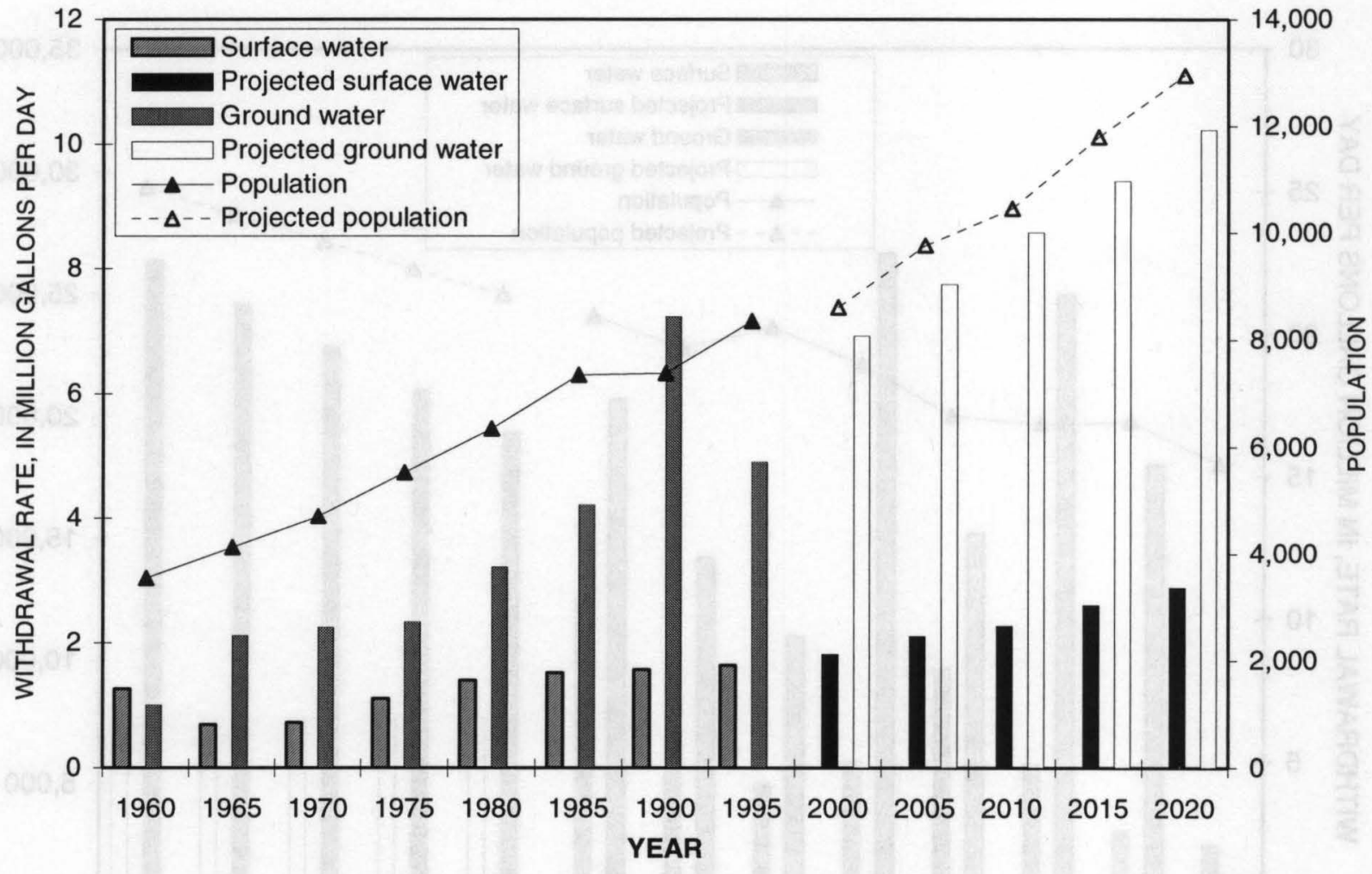

Figure 1. Historical and projected population and water-withdrawal rates, 1960-2020, for Ascension Parish within the Barataria-Terrebonne Basins, Louisiana. 


\section{Assumption Parish}

All of Assumption Parish is located within the boundaries of the Barataria-Terrebonne Basins. In 1995 , surface-water withdrawal rates accounted for $18 \mathrm{Mgal} / \mathrm{d}$ or 62 percent of the total withdrawal rates of $29 \mathrm{Mgal} / \mathrm{d}$. Total water-withdrawal rates increased 869 percent since 1960 (fig. 2). Neither variable in the ground-water regression model met the 0.15 significance level, so the mean was used for the projections. Multiple regression was used for the predicted surface-water withdrawal rates. In 2020, the projected total water-withdrawal rate is $29 \mathrm{Mgal} / \mathrm{d}$ or an increase of 2.3 percent from the 1995 total water-withdrawal rate. The overall water-use trend from 1960-95 was uneven. The large fluctuation in surface-water withdrawal rates of $9.4 \mathrm{Mgal} / \mathrm{d}$ up to $22 \mathrm{Mgal} / \mathrm{d}$ from $1970-95$ is primarily due to an industrial facility. The facility used large amounts of surface water in some years and significantly reduced its surface-water withdrawals in other years. The industry closed in early 1996. Because of these large fluctuations and its closure, the industrial facility's water-use values were excluded from the multiple regression analysis. Consequently, the projected withdrawal rates for the year 2000 show a small decrease, then a steady small increase in withdrawal rates after 2000 .

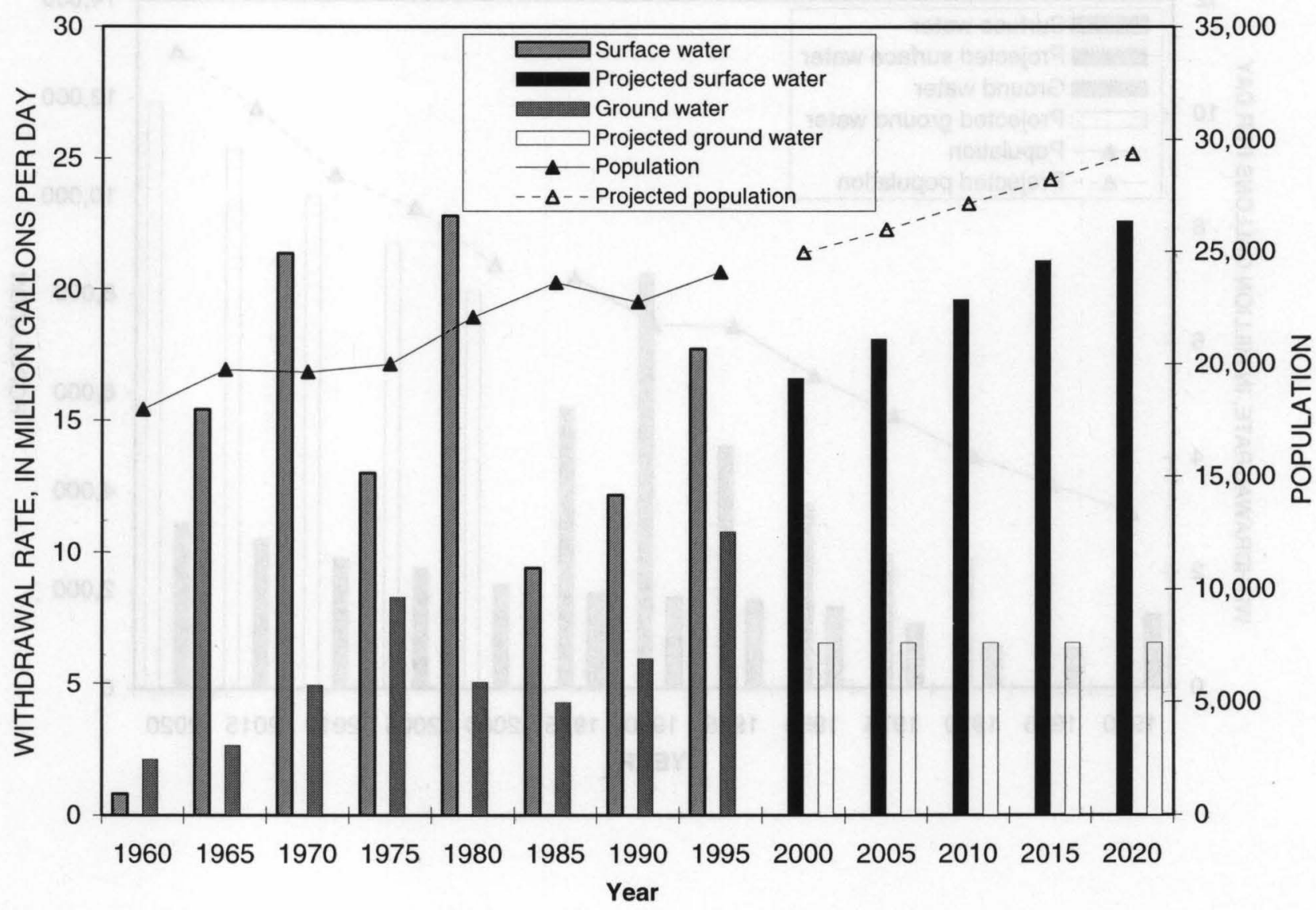

Figure 2. Historical and projected population and water-withdrawal rates, 1960-2020, for Assumption Parish within the Barataria-Terrebonne Basins, Louisiana. 


\section{Iberville Parish}

Fifty-seven percent of Iberville Parish is located within the boundaries of the Barataria-Terrebonne Basins. In 1995, total water-withdrawal rates in Iberville Parish within the study area were $24 \mathrm{Mgal} / \mathrm{d}$. Surface-water withdrawal rates accounted for 42 percent or $10 \mathrm{Mgal} / \mathrm{d}$ of that amount. Total waterwithdrawal rates increased 120 percent from 1960 to 1995 (fig. 3). In 1995, more than 54 percent of the ground- and surface-water withdrawal rates within the study area were from industrial facilities. The projection for total water-withdrawal rates in 2020 is $40 \mathrm{Mgal} / \mathrm{d}$ or an increase of 64 percent from 1995 . Because there is no significant historic trend, neither variable in the ground- or surface-water regression model met the 0.15 significance level, and the means were used for the projected withdrawal rates. The large increase in surface-water withdrawal rates in 1990 was due to higher per-acre application rates for crawfish farming. The decrease in ground-water withdrawal rates from 1990-95 was due in part to decreased use by one industrial facility. The decrease in surface-water withdrawal rates was due in part to a decrease in industrial withdrawal rates and lower per-acre application rates (modified in 1995) for crawfish farming.

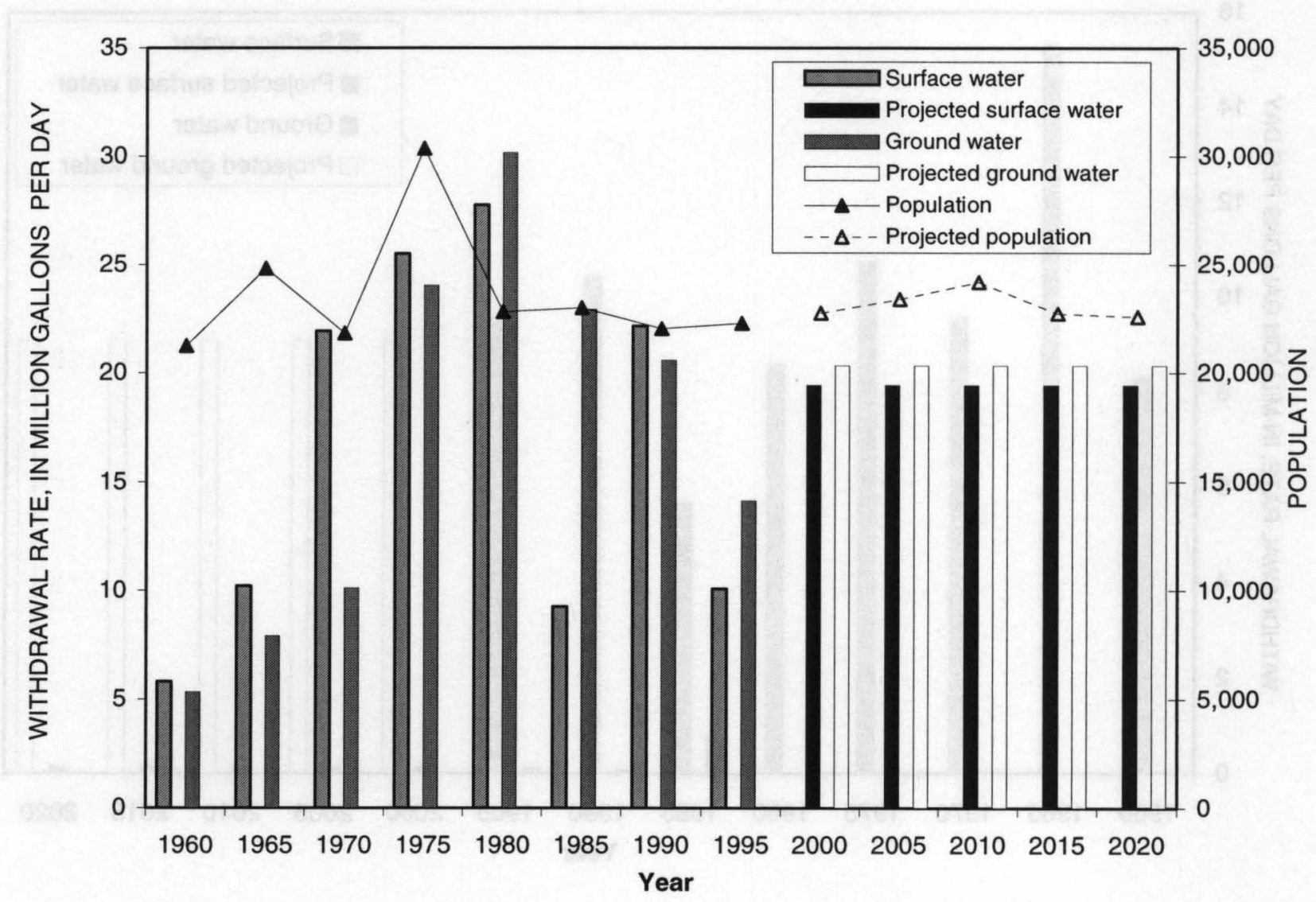

Figure 3. Historical and projected population and water-withdrawal rates, 1960-2020, for Iberville Parish within the Barataria-Terrebonne Basins, Louisiana. 


\section{Jefferson Parish}

Sixty-three percent of Jefferson Parish is located within the boundaries of the Barataria-Terrebonne Basins. Although the Mississippi River supplies more than 99 percent of all water used in Jefferson Parish, ground water accounts for the majority of water pumped in the part of the parish that lies within the study area. In 1995, surface-water withdrawal rates accounted for only about 1 percent or $0.09 \mathrm{Mgal} / \mathrm{d}$ of the total withdrawal rates of $9.6 \mathrm{Mgal} / \mathrm{d}$. Total water-withdrawal rates increased 12 percent from 1960-95 (fig. 4). Most of the ground- and surface-water withdrawal rates, 63 percent, were from industrial facilities in 1995. Because no public-supply withdrawal rates were reported in the part of this parish that is in the study area, population could not be used in the regression model. The date variable in the ground- and surface-water regression models failed to meet the 0.15 significance level, and the means were used for the projected withdrawal rates. The projection for total water-withdrawal rates in 2020 is projected as $9.3 \mathrm{Mgal} / \mathrm{d}$, a slight decrease of 3 percent from the 1995 total water-withdrawal rates for the parish.

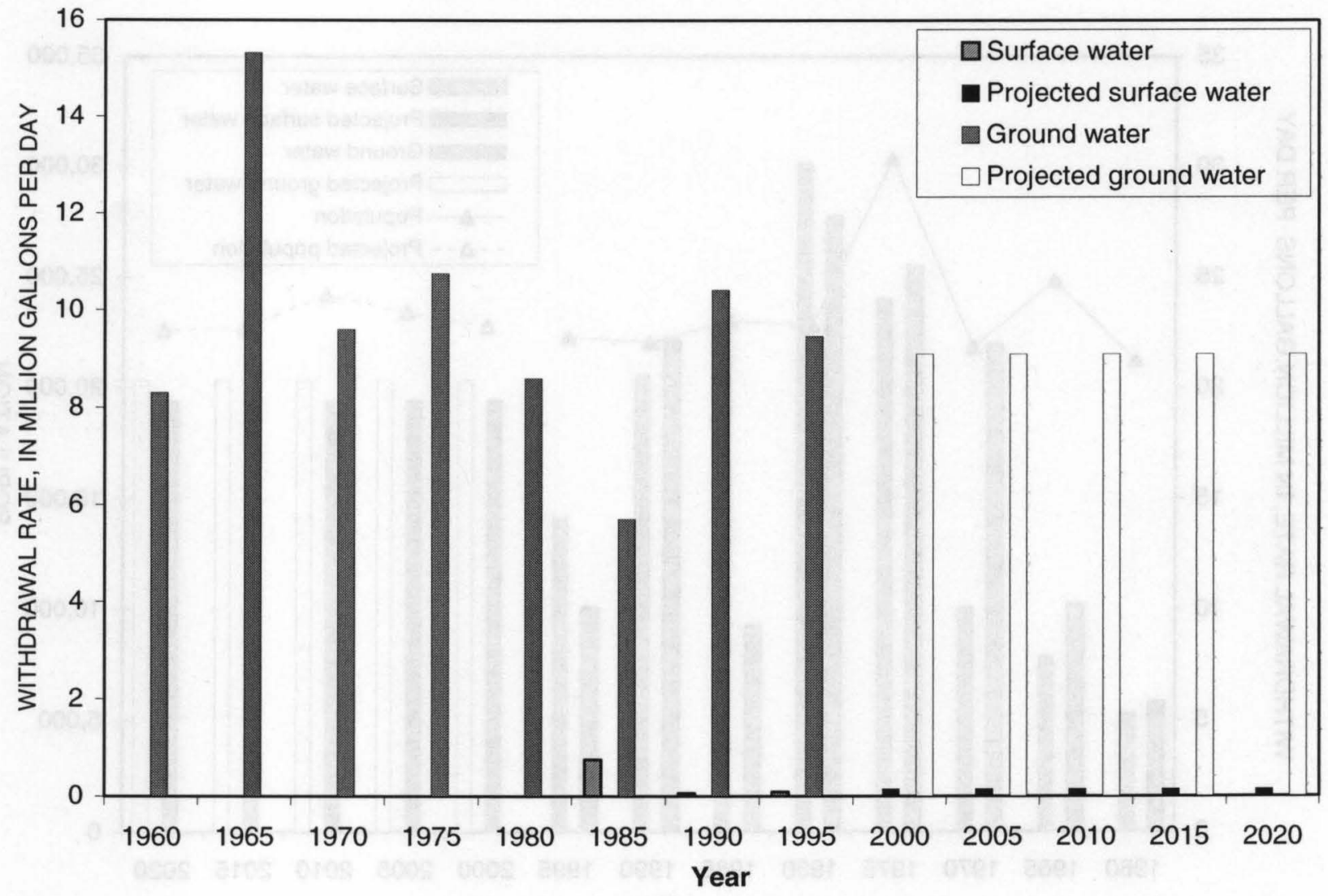

Figure 4. Historical and projected water-withdrawal rates, 1960-2020, for Jefferson Parish within the Barataria-Terrebonne Basins, Louisiana. 


\section{Lafourche Parish}

All of Lafourche Parish is located within the boundaries of the Barataria-Terrebonne Basins. Lafourche Parish also had the largest water-withdrawal rates within the basins, $36 \mathrm{Mgal} / \mathrm{d}$ in 1995 . Of that amount, surface-water withdrawal rates accounted for 96 percent or $35 \mathrm{Mgal} / \mathrm{d}$. In 1995, more than 55 percent of ground- and surface-water withdrawal rates within the study area were from public water suppliers. Total water-withdrawal rates increased 327 percent from 1960 to 1995 (fig. 5). Multiple regression was used for the projected ground-water withdrawal rates. Neither variable in the surface-water regression model met the 0.15 significance level, and the mean was used for the projected withdrawal rates. Total water-withdrawal rates in 2020 is projected to be $39 \mathrm{Mgal} / \mathrm{d}$, an increase of 8 percent from the 1995 total water-withdrawal rates. The large increase in surface-water withdrawal rates in 1990 and subsequent decrease in 1995 were due to higher per-acre application rates (modified in 1995) for crawfish farming.

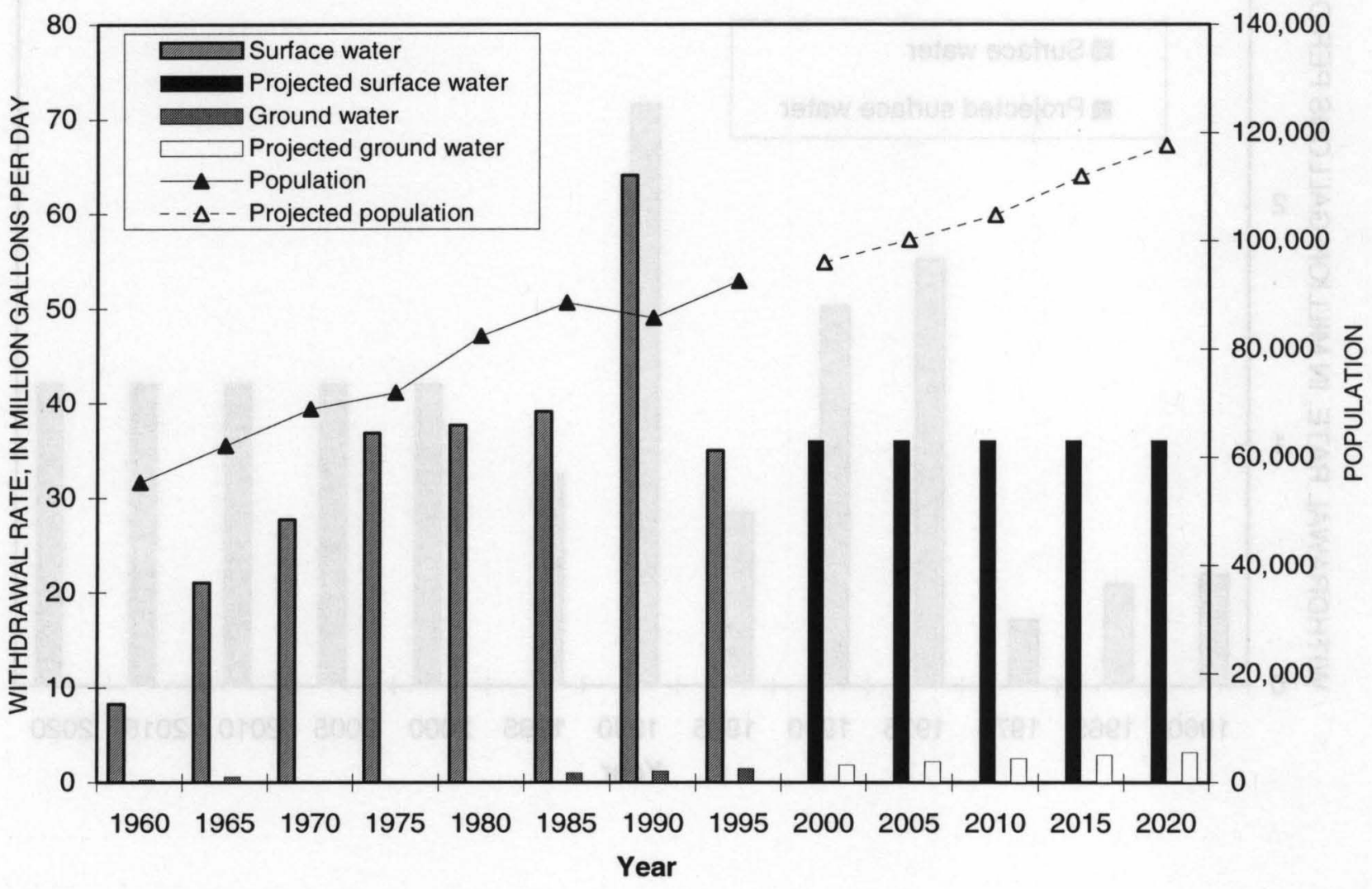

Figure 5. Historical and projected population and water-withdrawal rates, 1960-2020, for Lafourche Parish within the Barataria-Terrebonne Basins, Louisiana. 


\section{Plaquemines Parish}

Thirty percent of Plaquemines Parish is located within the boundaries of the Barataria-Terrebonne Basins. In 1995, surface-water withdrawal rates for livestock and aquaculture accounted for 100 percent or $0.82 \mathrm{Mgal} / \mathrm{d}$ of the total withdrawal rates in the part of the parish within the study area. Aquaculture withdrawal rates accounted for 91 percent of that total. For this reason, population could not be used in the regression model. Because the date variable in the surface-water regression model failed to meet the 0.15 significance level, the mean was used for the projected withdrawal rates. Total water-withdrawal rates increased 77 percent from 1960 to 1995 (fig. 6). The projection for total water-withdrawal rates in 2020 is $1.3 \mathrm{Mgal} / \mathrm{d}$, an increase of 53 percent from the 1995 total water-withdrawal rates.

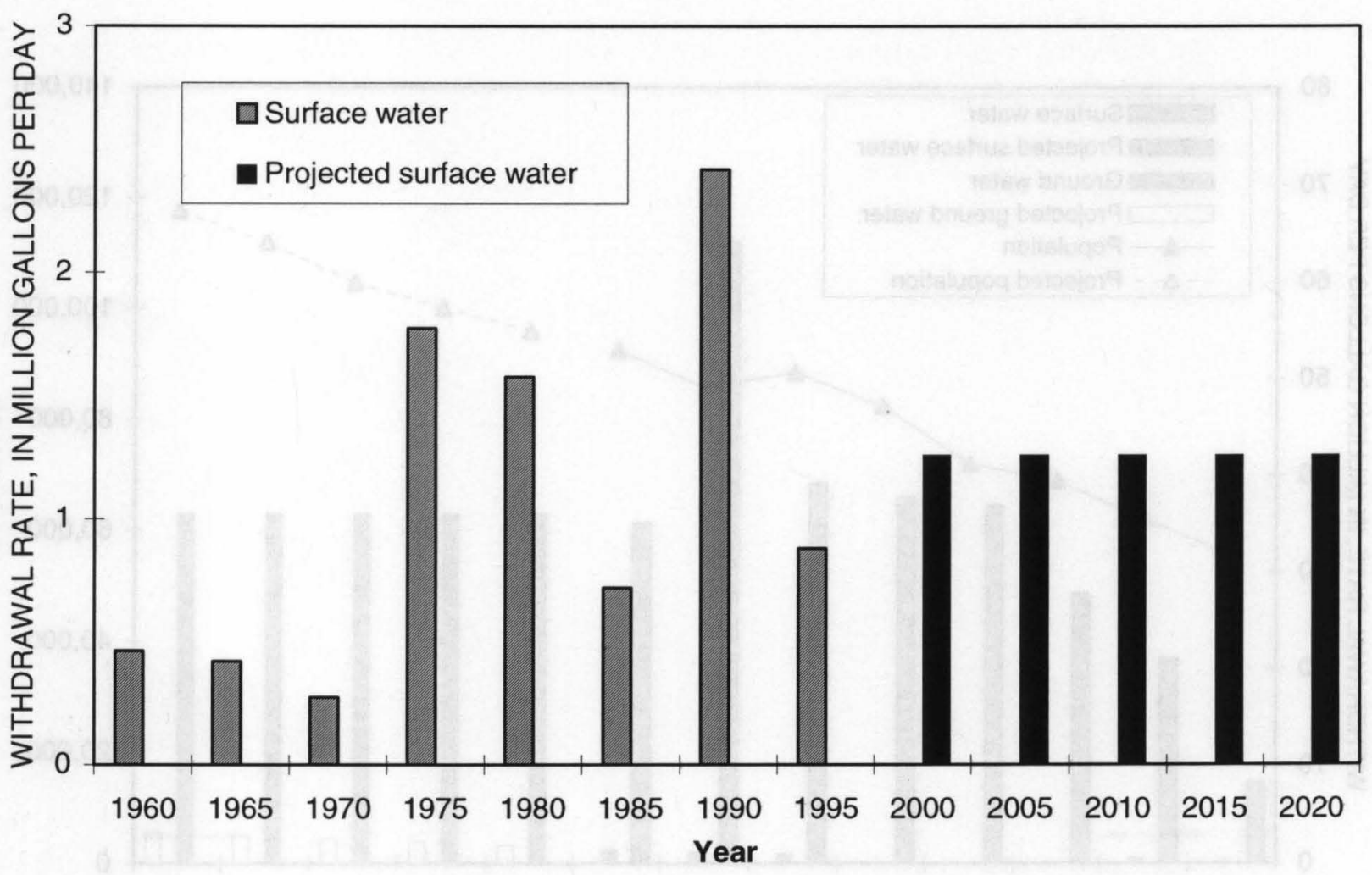

Figure 6. Historical and projected water-withdrawal rates, 1960-2020, for Plaquemines Parish within the Barataria-Terrebonne Basins, Louisiana. 


\section{Pointe Coupee Parish}

Thirty-nine percent of Pointe Coupee Parish is located within the boundaries of the BaratariaTerrebonne Basins. Ground water is the primary source of water. In 1995, surface-water withdrawal rates accounted for only $2.1 \mathrm{Mgal} / \mathrm{d}$ or 19 percent of the total withdrawal rates of $11 \mathrm{Mgal} / \mathrm{d}$. Most of those withdrawal rates are from miscellaneous streams and are used for aquaculture and irrigation. Total waterwithdrawal rates increased 490 percent from 1960 to 1995 (fig. 7). Multiple regression was used for the projected ground-water withdrawal rates. Neither variable in the surface-water regression model met the 0.15 significance level, and the mean was used for the projected withdrawal rates. The projection for total water-withdrawal rates in 2020 is $18 \mathrm{Mgal} / \mathrm{d}$ or an increase of 58 percent from the 1995 total waterwithdrawal rates. The large increase in ground-water withdrawal rates in 1990 was due in part to an increase in withdrawal rates for crawfish farming. Ground-water withdrawal rates at a fossil-fuel plant located in this parish are included. Withdrawal rates of Mississippi River water at this plant are not included.

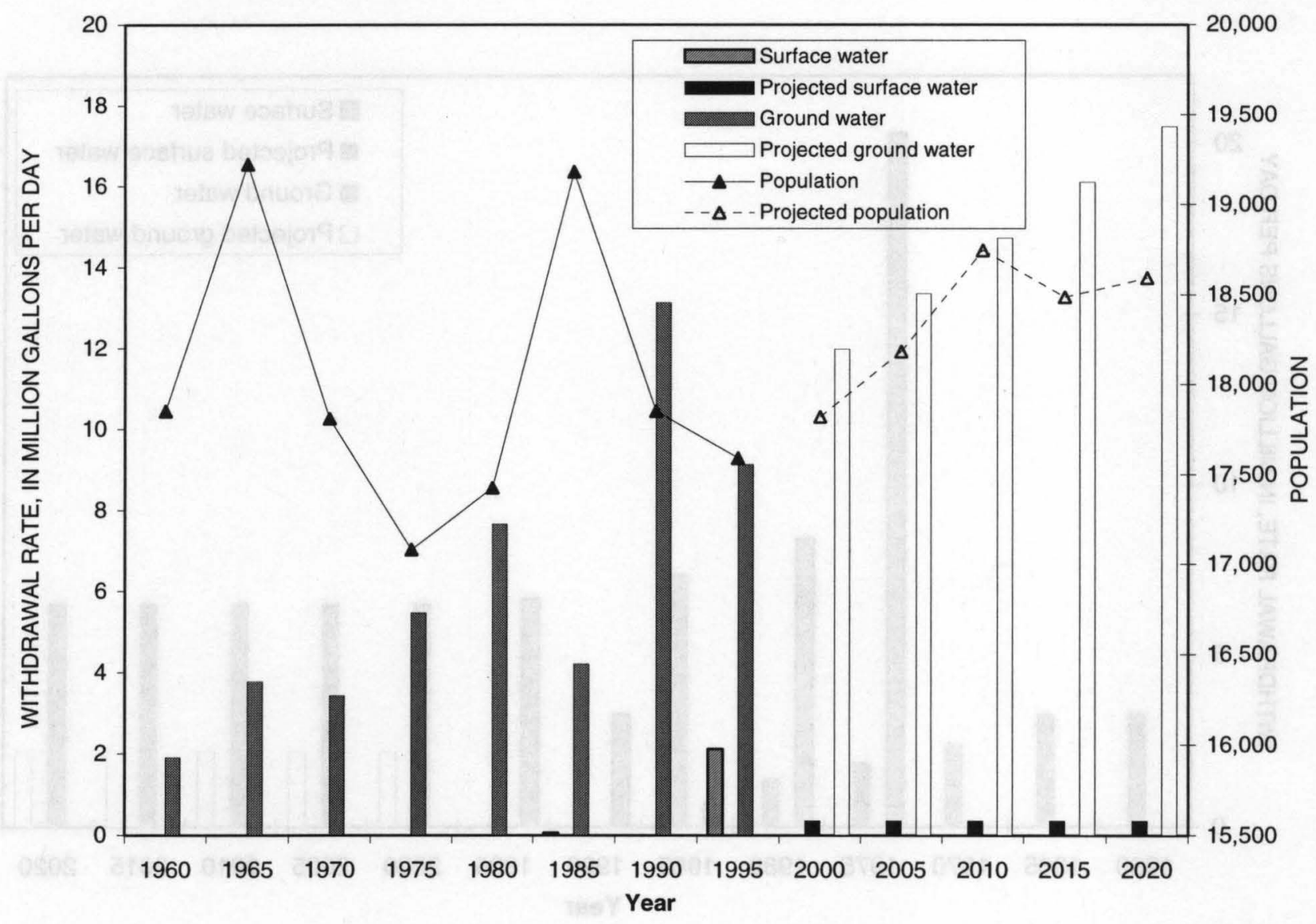

Figure 7. Historical and projected population and water-withdrawal rates, 1960-2020, for Pointe Coupee Parish within the Barataria-Terrebonne Basins, Louisiana. 


\section{St. Charles Parish}

Sixty percent of St. Charles Parish is located within the boundaries of the Barataria-Terrebonne Basins. Surface water has been the predominate source of water for the parish within the basins since 1990. In 1995, surface-water withdrawal rates accounted for $6.6 \mathrm{Mgal} / \mathrm{d}$ or 99 percent of the total withdrawal rates of $6.7 \mathrm{Mgal} / \mathrm{d}$. Of the total ground- and surface-water withdrawal rates within the study area in 1995, almost 88 percent were from industrial facilities. Because no public-supply withdrawal rates were reported in the part of this parish that is in the study area, population could not be used in the regression model. The date variable in the ground- and surface-water regression models failed to meet the 0.15 significance level, and the means were used for the projected withdrawal rates. Total water-withdrawal rates increased 102 percent from 1960 to 1995 (fig. 8). The projection for total water-withdrawal rates in 2020 is $8.8 \mathrm{Mgal} / \mathrm{d}$, an increase of 30 percent from the 1995 total water-withdrawal rates. The large surface-water withdrawal rates in 1975 are due largely to one industrial facility. This facility did not report withdrawal rates in 1985 or 1990.

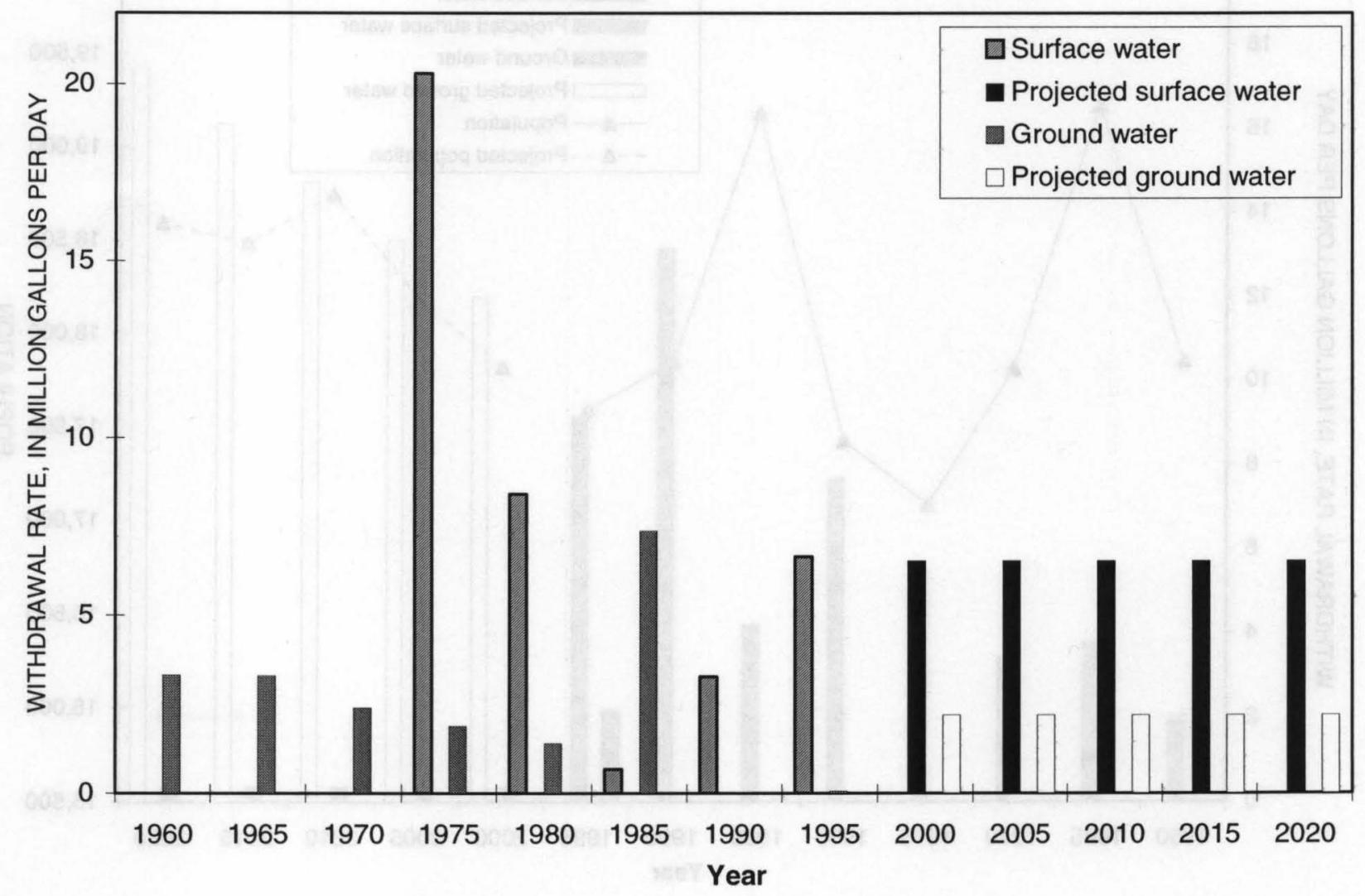

Figure 8. Historical and projected water-withdrawal rates, 1960-2020, for St. Charles Parish within the Barataria-Terrebonne Basins, Louisiana. 


\section{St. James Parish}

Forty-one percent of St. James Parish is located within the boundaries of the Barataria-Terrebonne Basins. In 1995, surface-water withdrawal rates for aquaculture accounted for $8.0 \mathrm{Mgal} / \mathrm{d}$ or 88 percent of the total withdrawal rates of $9.1 \mathrm{Mgal} / \mathrm{d}$ within the study area. Total water-withdrawal rates increased 82 percent from 1960 to 1995 (fig. 9). There were no surface-water withdrawal rates in 1985 . Because no public-supply withdrawal rates were reported in the part of this parish that is in the study area, population could not be used in the regression model. The date variable in the ground- and surface-water regression models failed to meet the 0.15 significance level, and the means were used for the projected withdrawal rates. Total water-withdrawal rates in 2020 are projected as $21 \mathrm{Mgal} / \mathrm{d}$, an increase of 130 percent from 1995. The large increase in surface-water withdrawal rates in 1990 was due to higher per-acre application rates (modified in 1995) for crawfish farming.

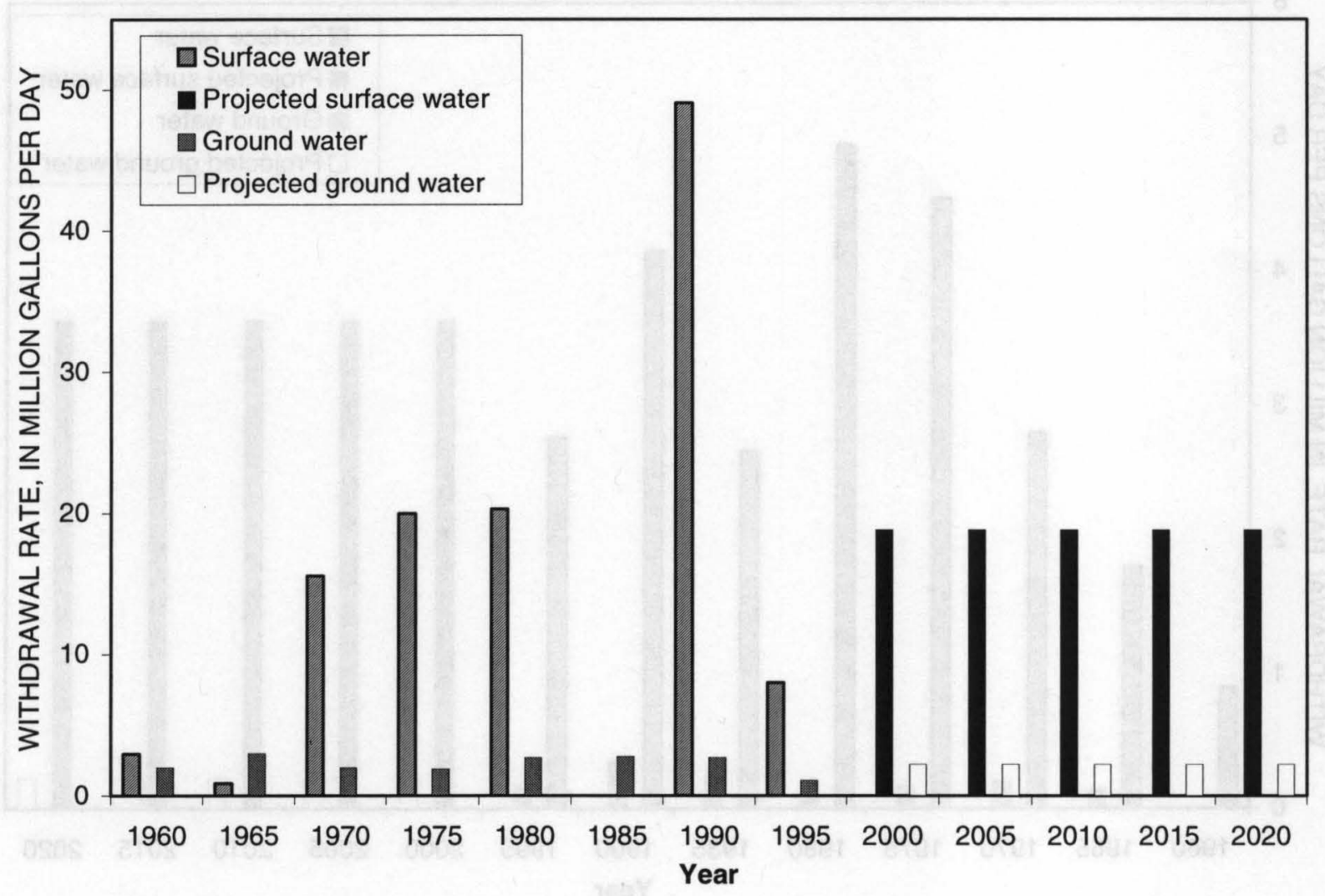

Figure 9. Historical and projected water-withdrawal rates, 1960-2020, for St. James Parish within the Barataria-Terrebonne Basins, Louisiana. 


\section{St. John the Baptist Parish}

Seventeen percent of St. John the Baptist Parish is located within the boundaries of the BaratariaTerrebonne Basins. In 1995, surface-water withdrawal rates accounted for almost all of the withdrawal rates in the parish within the basins, or 94 percent $(2.8 \mathrm{Mgal} / \mathrm{d})$ of the total withdrawal rates. Of the total surfacewater withdrawal rates for 1995 , about 96 percent of the withdrawal rates were from industrial facilities within the study area, with the remaining withdrawal rates for alligator farming. Total water-withdrawal rates increased 220 percent from 1960 to 1995 (fig. 10). Because no public-supply withdrawal rates were reported in the part of this parish that is in the study area, population could not be used in the regression model. The date variable in the ground- and surface-water regression models failed to meet the 0.15 significance level, and the means were used for the projected withdrawal rates. The projection for total water- withdrawal rates in 2020 is $3.9 \mathrm{Mgal} / \mathrm{d}$, an increase of 32 percent from 1995.

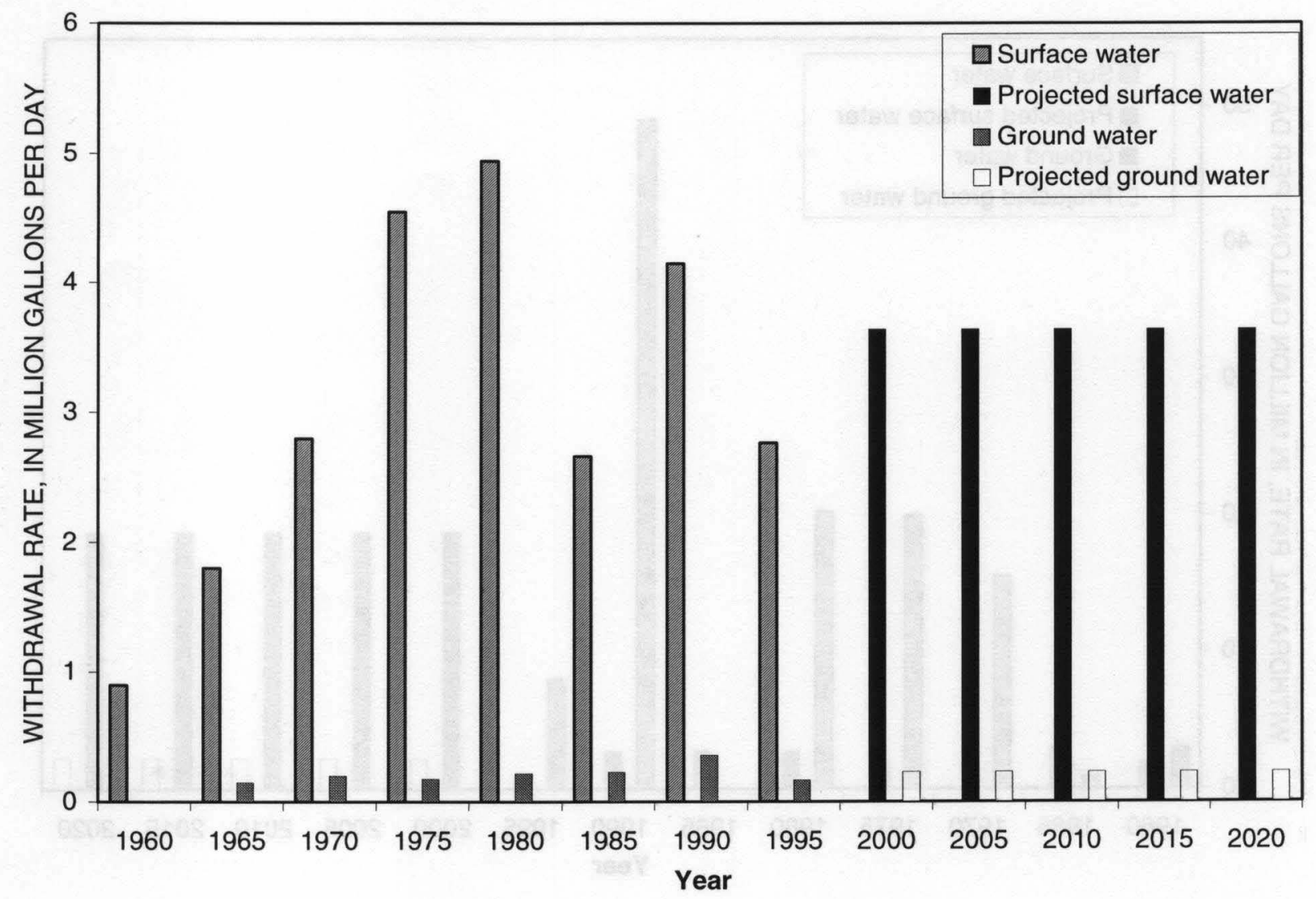

Figure 10. Historical and projected water-withdrawal rates, 1960-2020, for St. John the Baptist Parish within the Barataria-Terrebonne Basins, Louisiana. 


\section{St. Mary Parish}

About 7.1 percent of St. Mary Parish is located within the boundaries of the Barataria-Terrebonne Basins. Surface water is the predominant source of freshwater in this parish within the basins. In 1995, surface-water withdrawal rates accounted for almost $14 \mathrm{Mgal} / \mathrm{d}$ or 100 percent of the withdrawal rates in the parish within the basins. Of that amount, one industrial facility withdrew more than 92 percent of the total withdrawal rates in the parish within the study area. Surface-water withdrawal rates increased 790 percent from 1960 to 1995 (fig. 11). There were no withdrawal rates reported in the parish within the study area in 1985. Neither variable in the surface-water regression model met the 0.15 significance level, and the mean was used for the projected withdrawal rates. The projection for total surface-water withdrawal rates in 2020 is $4.3 \mathrm{Mgal} / \mathrm{d}$, a decrease of 69 percent from 1995 . Should the industrial facility, which has been closed since 1995 , begin operations, this amount would sharply increase.

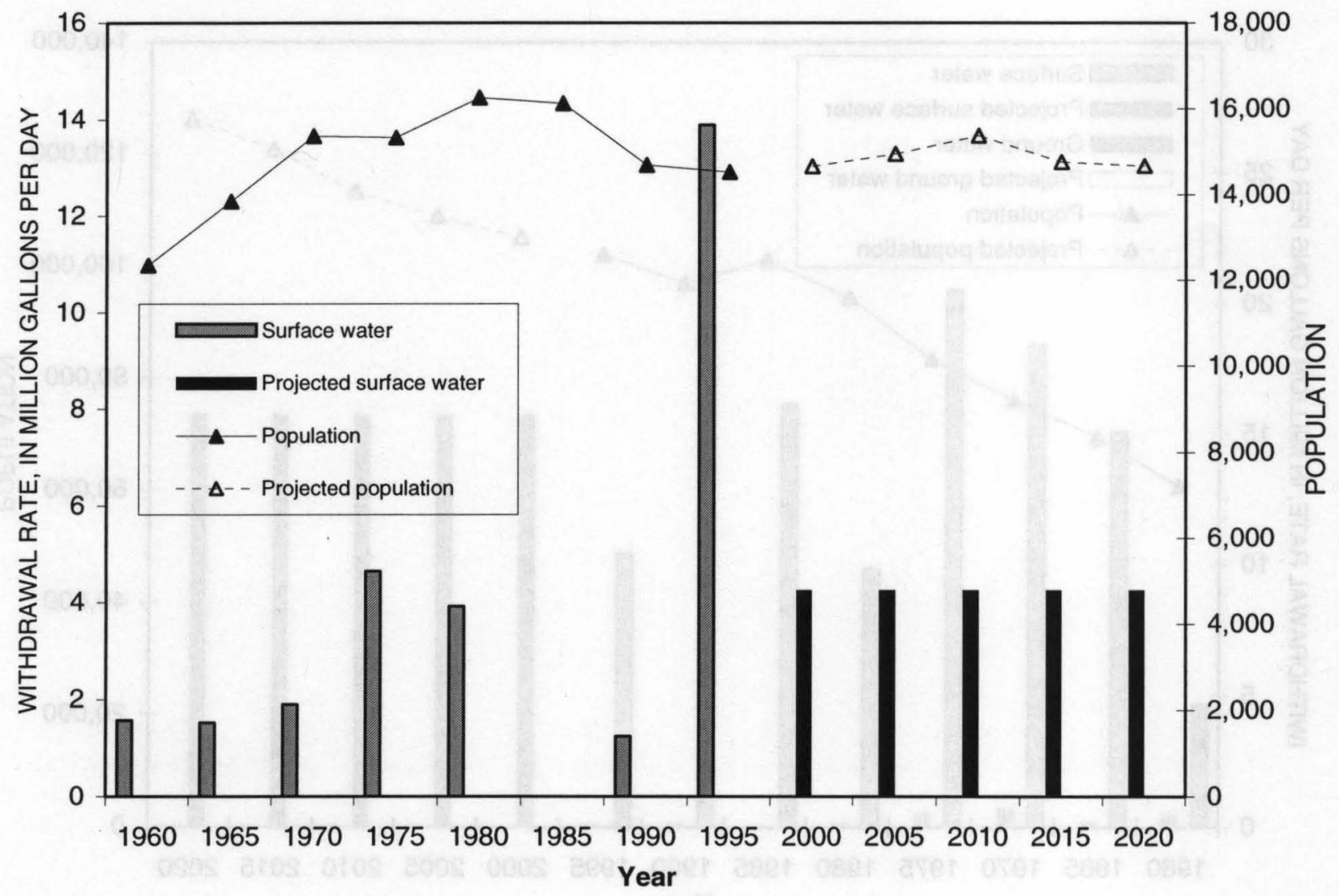

Figure 11. Historical and projected population and water-withdrawal rates, 1960-2020, for St. Mary Parish within the Barataria-Terrebonne Basins, Louisiana. 


\section{Terrebonne Parish}

All of Terrebonne Parish is located within the boundaries of the Barataria-Terrebonne Basins. In 1995, surface-water withdrawal rates accounted for about 99 percent or $10 \mathrm{Mgal} / \mathrm{d}$ of the total withdrawal rates. Withdrawal rates from public water suppliers accounted for almost 46 percent of the total withdrawal rates, followed by withdrawal rates for alligator farming and industry. Total water-withdrawal rates increased over 100 percent from 1960 to 1995 (fig. 12). Multiple regression. was used for the projected ground-water withdrawal rates. Ground-water use has considerably declined since 1970. Based on visual inspection of the regression results which reduced the projected ground-water withdrawal to a negative number by 2000 , the average of the 1980-95 values was used for the projected ground-water withdrawal rates. There is no significant trend in water use from 1960-95. Neither variable in the surface-water regression model met the 0.15 significance level, and the mean was used for the projected surface-water withdrawal rates. The projection for total water-withdrawal rates in 2020 is $16 \mathrm{Mgal} / \mathrm{d}$, an increase of 50 percent from 1995.

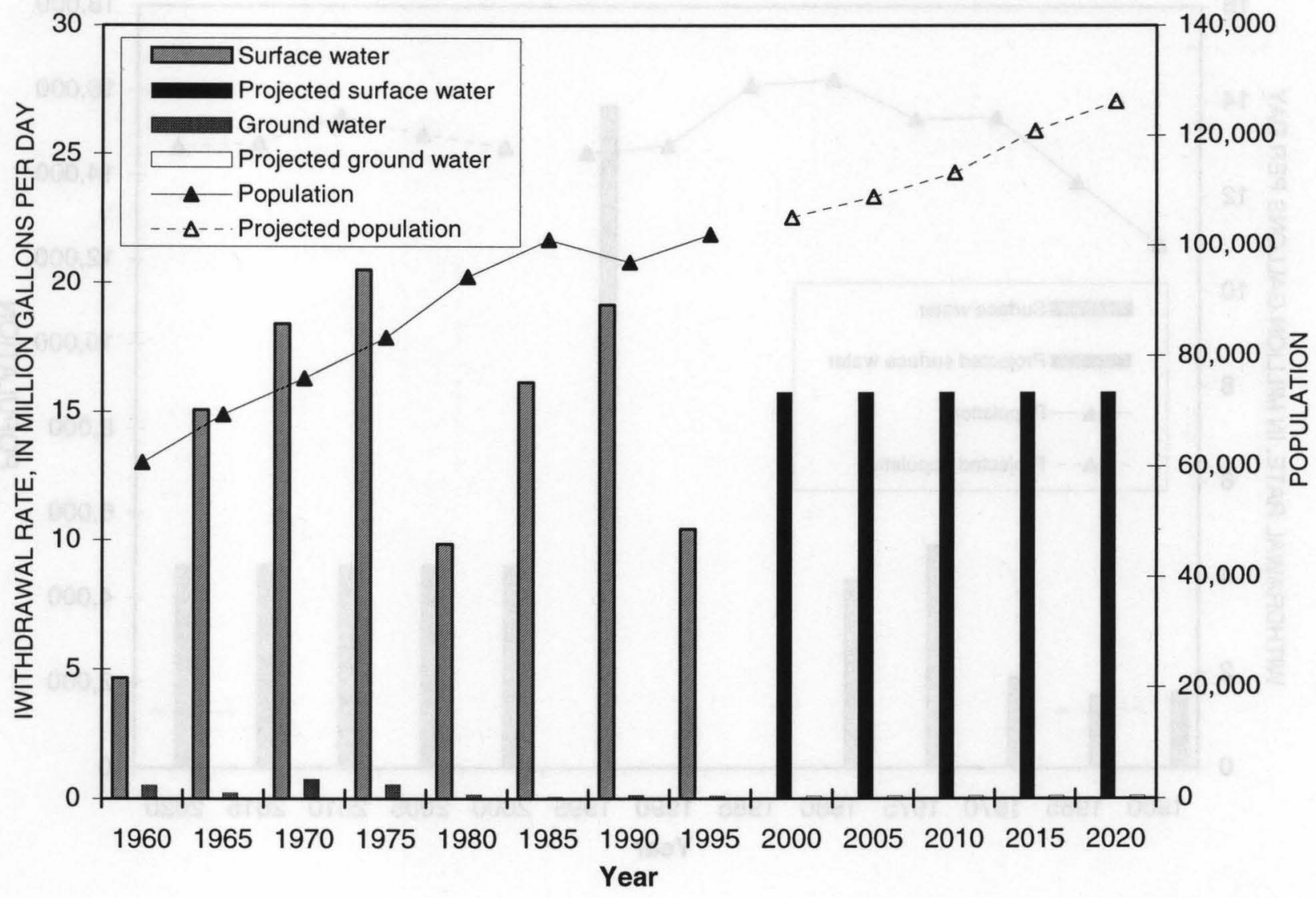

Figure 12. Historical and projected population and water-withdrawal rates, 1960-2020, for Terrebonne Parish within the Barataria-Terrebonne Basins, Louisiana. 


\section{West Baton Rouge Parish}

Seventy-one percent of West Baton Rouge Parish is located within the boundaries of the BaratariaTerrebonne Basins. Ground water is the predominate source of water in this parish within the basins. In 1995 , surface-water withdrawal rates accounted for only 4.4 percent or $0.53 \mathrm{Mgal} / \mathrm{d}$ of the total withdrawal rates of $12 \mathrm{Mgal} / \mathrm{d}$. Most of the withdrawal rates in the parish within the study area were from public water suppliers and industrial facilities in 1995. Total water-withdrawal rates increased 460 percent from 1960 to 1995 (fig. 13). Multiple regression was used for the projected ground-water withdrawal rates. Neither variable in the surface-water regression model met the 0.15 significance level, and the mean was used for the projected withdrawal rates. The projection for total water-withdrawal rates in 2020 is $20 \mathrm{Mgal} / \mathrm{d}$, an increase of 70 percent from 1995.

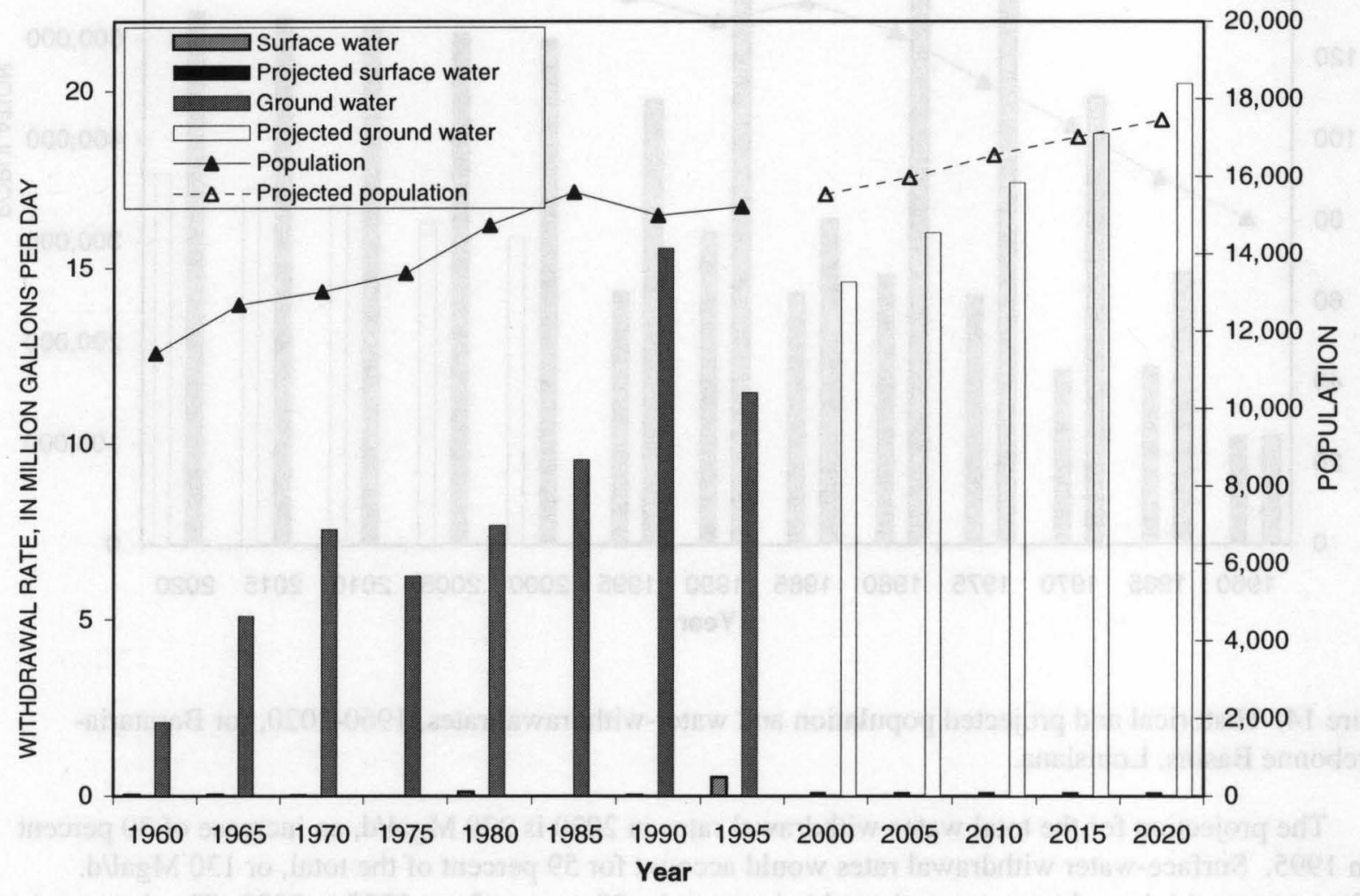

Figure 13. Historical and projected population and water-withdrawal rates, 1960-2020, for West Baton Rouge Parish within the Barataria-Terrebonne Basins, Louisiana. 


\section{Trend and Projection for Barataria-Terrebonne Basins}

In 1995, total water-withdrawal rates of $170 \mathrm{Mgal} / \mathrm{d}$ in the basins increased 221 percent since 1960 (fig. 14). Surface-water withdrawal rates increased by 310 percent, and ground-water withdrawal rates increased by 133 percent.

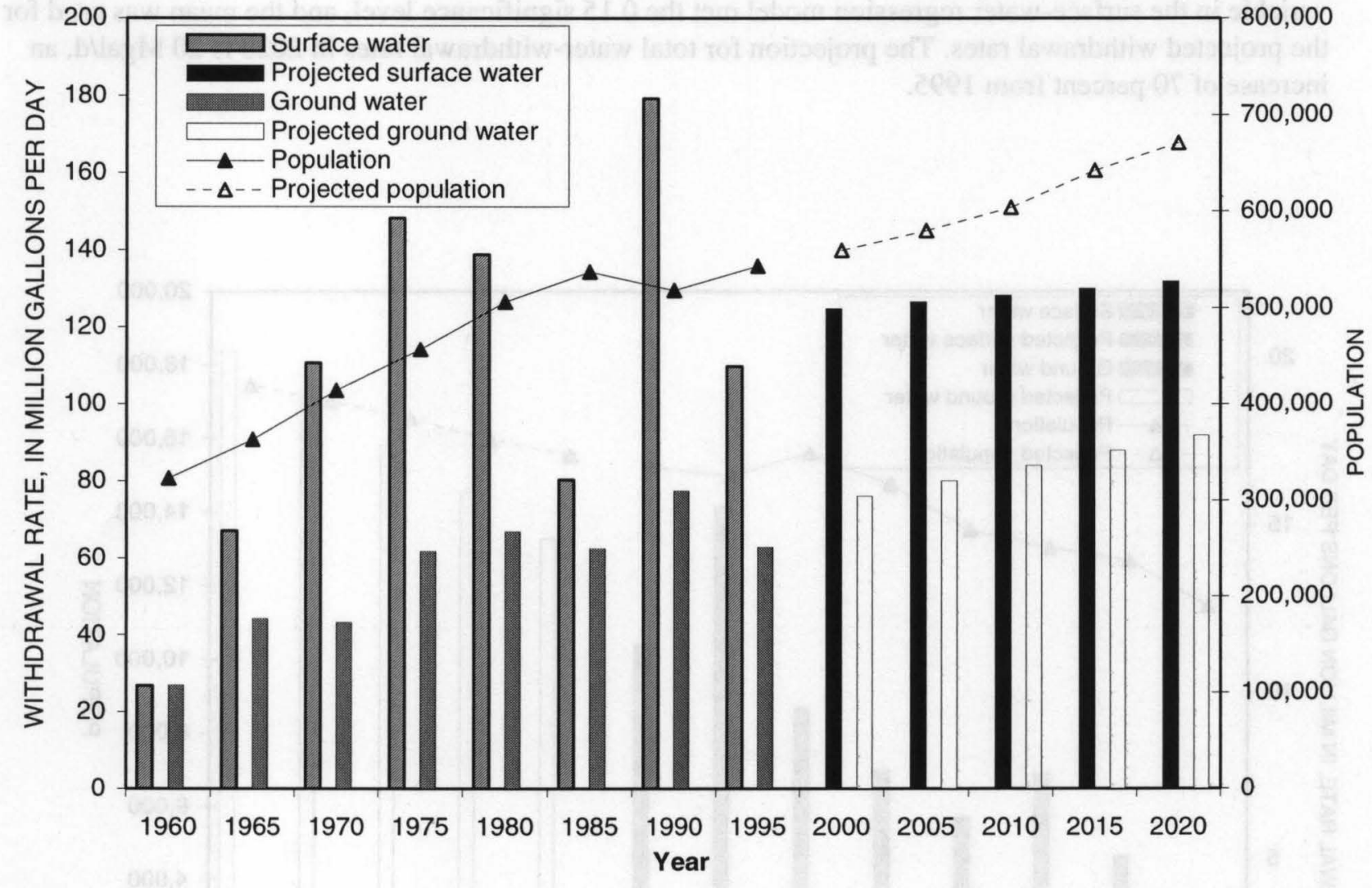

Figure 14. Historical and projected population and water-withdrawal rates, 1960-2020, for BaratariaTerrebonne Basins, Louisiana.

The projection for the total water-withdrawal rates in 2020 is $220 \mathrm{Mgal} / \mathrm{d}$, an increase of 30 percent from 1995. Surface-water withdrawal rates would account for 59 percent of the total, or $130 \mathrm{Mgal} / \mathrm{d}$. Surface-water withdrawal rates are projected to increase by 20 percent from 1995 to 2020 . The decrease in surface-water withdrawal rates in 1985 is due in part to a decrease in industrial withdrawal rates for that year. The increase in withdrawal rates in 1990 and subsequent decrease in 1995 are mainly due to the different methods in calculating the amount of water used in crawfish farming. Past trends of ground-and surface-water use show an uneven pattern with a net increase over time. Taking this into account, the 30 percent increase in total withdrawal rates from 1995 to 2020 within the basins would probably follow an uneven pattern. Total ground-and surface-water withdrawal rates and population for each parish within the basins are included in table 5, from 1960 through 1995, with projected ground- and surface-water withdrawal rates from 2000 through 2020. 
Table 5. Ground-and surface-water withdrawal rates and population in the Barataria-Terrebonne Basins, in Louisiana, 1960-95, and projected ground-and surface-water withdrawal rates and population through 2020

\begin{tabular}{cccc}
\hline Year & \multicolumn{2}{c}{ Withdrawal rates (million gallons per day) } & $\begin{array}{c}\text { Population } \\
\text { (thousands) }\end{array}$ \\
\cline { 2 - 4 } & Surface water & Ground water & 3,228 \\
\hline 1960 & 26.77 & 26.96 & 3,628 \\
1965 & 67.09 & 44.25 & 4,145 \\
1975 & 110.65 & 43.35 & 4,565 \\
1980 & 148.25 & 61.73 & 5,062 \\
1985 & 138.80 & 66.96 & 5,372 \\
1990 & 80.33 & 62.45 & 5,181 \\
1995 & 179.33 & 77.45 & 5,431 \\
2000 & 109.77 & 62.88 & 5,597 \\
2005 & 124.88 & 76.13 & 5,801 \\
2010 & 126.67 & 80.07 & 6,040 \\
2015 & 128.34 & 84.01 & 6,425 \\
2020 & 130.17 & 87.97 & 6,712 \\
\hline
\end{tabular}

\section{QUALITY OF FRESH SURFACE-WATER RESOURCES}

Bayou Lafourche receives almost all of its discharge from the Mississippi River. Therefore, an understanding of Mississippi River water quality and its relation to Bayou Lafourche is important. From 2,500 to 800 years ago, the main channel of the Mississippi River flowed down Bayou Lafourche (Spearing, 1995, p. 76). The Mississippi River retained a small connection with the bayou until it was closed off in 1904 to lessen the threat of flooding. In 1997, several hundred cubic feet per second of Mississippi River water were pumped from the river by the BLFWD to maintain flow in the bayou. (Kirk Cheramie, Bayou Lafourche Freshwater District, written commun., 1997).

The USGS and the Louisiana Department of Environmental Quality (DEQ) have compiled an extensive data base of water-quality information from the lower Mississippi River. Also, DEQ has compiled a water-quality assessment of all major water bodies in Louisiana (Louisiana Department of Environmental Quality, 1996). In the category "Degree of Support," the 68-mile reach of Bayou Lafourche from Donaldsonville to Larose was determined to be "Not Supporting" for primary contact recreation (swimming), secondary contact recreation (boating), and fish and wildlife propagation. Suspected sources include minor industrial and municipal point sources, nonpoint agricultural runoff, spills, and improperly maintained septic tanks (Louisiana Department of Environmental Quality, 1996, p. A-10). The remaining reach of the bayou was assessed as "Partially Supporting" its designated uses. Other major waterways in the Barataria-Terrebonne Basins assessed as "Not Supporting" include Bayou Terrebonne, Company Canal, parts of the GIWW, and the upper 20 miles of Bayou Grand Caillou. 


\section{Potential Water-Quality Problems Concerning Diversion of Water from the Mississippi River}

Bayou Lafourche is being evaluated as a means of conveying freshwater from the Mississippi River to coastal Louisiana wetlands south of Larose (fig. 15) as a major component of Louisiana marsh restoration efforts. Various scenarios under study include Bayou Lafourche conveying an additional 500 to 700 cubic feet per second of freshwater. However, there is a widespread public perception that the river is highly contaminated by a wide variety of toxic compounds, and that the adverse effects of these pollutants would outweigh the benefits of additional freshwater, sediments, and nutrients. In response to these needs and concerns, both the water quality and hydrology of the Bayou Lafourche and GIWW systems have received increased monitoring by Federal agencies and universities. Discharge measurements conducted by the USGS in cooperation with the COE during 1995-97 have shown that the GIWW, which crosses Bayou Lafourche at Larose, has carried as much as 4,000 cubic feet per second of freshwater eastward across Bayou Lafourche. This flow is driven in large part by a favorable hydraulic gradient in the GIWW from west to east, and controlled by the amount of water flowing down the Atchafalaya River Basin. This flow is seasonal.

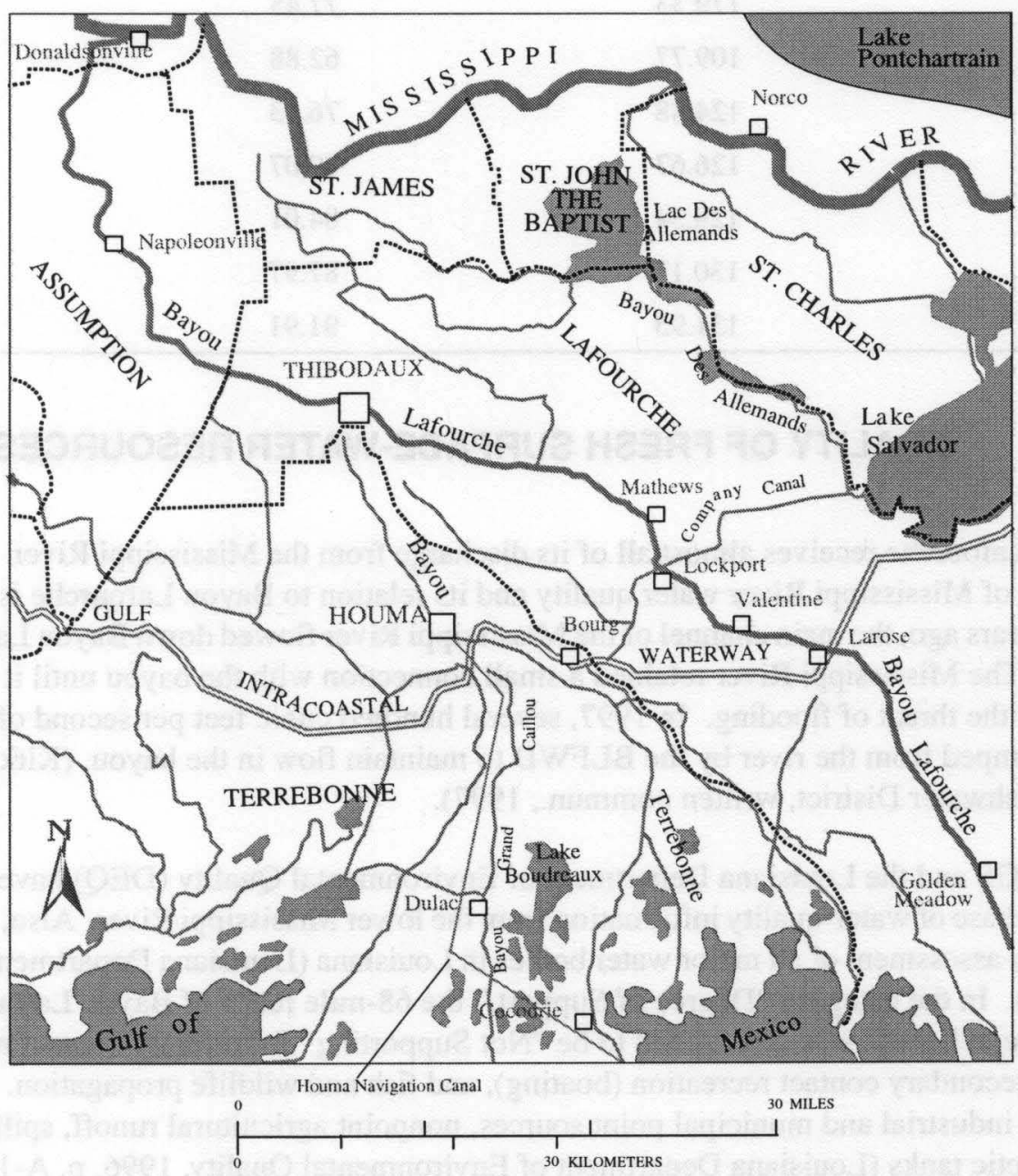

Figure 15. Bayou Lafourche area, Louisiana. 


\section{Toxic Compounds}

An analysis of the water quality of the lower Mississippi River in Louisiana using USGS and DEQ data indicates no constituents that exceed USEPA criteria for drinking water. The herbicide atrazine (fig. 16) is the only water-quality constituent that briefly exceeds the USEPA maximum contaminant level (MCL) of 3.0 micrograms per liter in the Mississippi River at Baton Rouge during the late spring and early summer. However, as the MCL is based on yearly averages of samples, the concentrations detected during the annual spring flush do not necessarily mean that the criterion is exceeded.

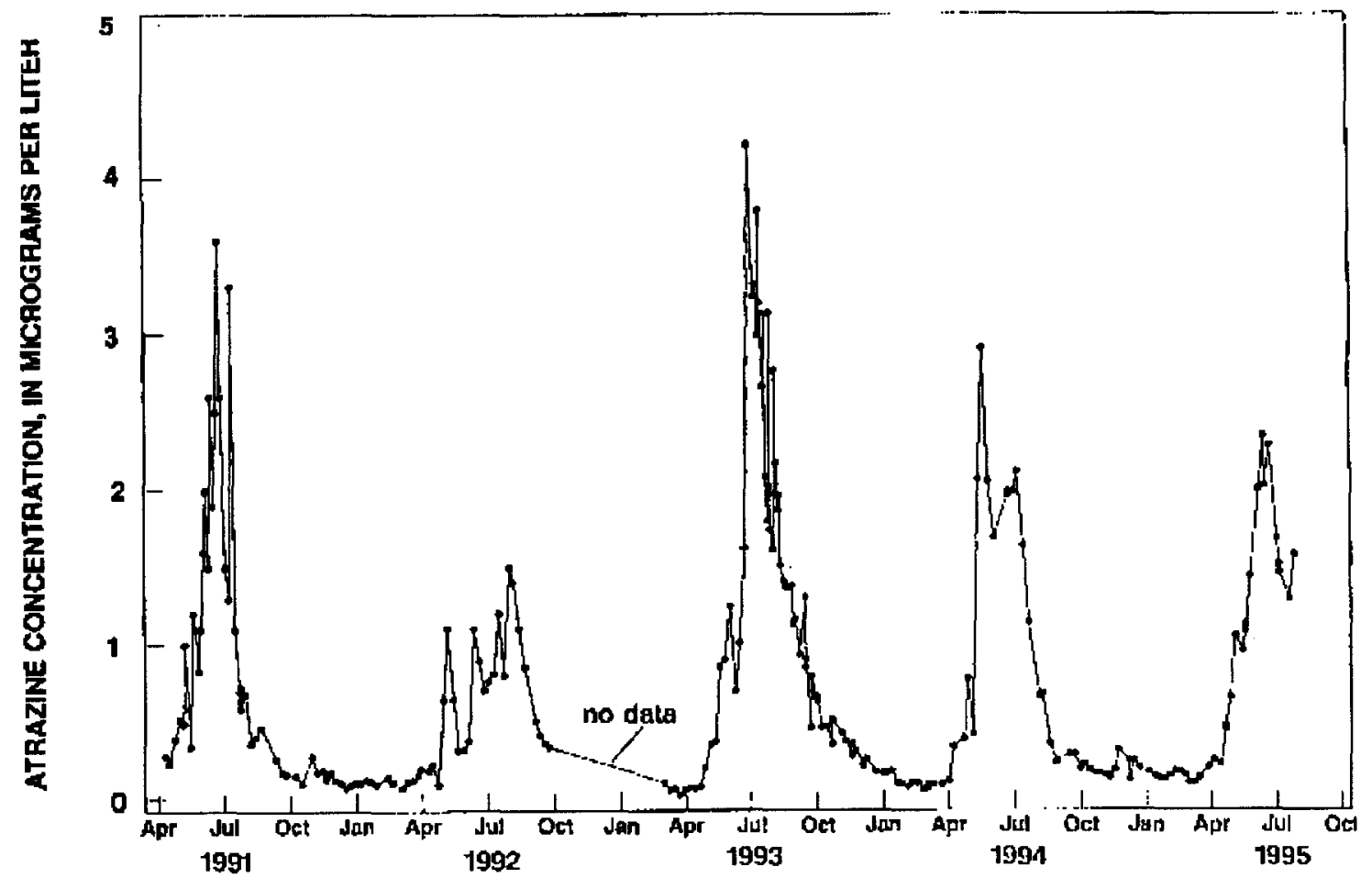

Figure 16. Atrazine concentration in the Mississippi River at Baton Rouge, Louisiana, 1991-95 (Goolsby and others, 1995).

Past studies (Wells, 1980; Demas and Curwick, 1987) indicate that trace metals are within recommended USEPA criteria for drinking water 99 percent of the time, and should not pose any serious ecological problems (Wells, 1980, p. 24). Trace elements have not become a problem primarily because of the physical and chemical characteristics of the river itself--the river has relatively coarse bottom sediments, a continuous downstream bed movement that does not allow trace elements to accumulate, and a relatively low amount of organic matter in the sediments.

In April 1991, a survey of trace elements in bottom material in Bayou Lafourche (McGee and Demcheck, 1995 ) indicated that arsenic, copper, lead, mercury, and zinc were found in concentrations that exceeded expected background concentrations between Donaldsonville and Golden Meadow. Arsenic concentrations ranged from 7.5 to $16 \mathrm{mg} / \mathrm{kg}$ (milligrams per kilogram) between Donaldsonville and Golden Meadow. In the same reach of the bayou, copper ranged from 18 to $53 \mathrm{mg} / \mathrm{kg}$; lead ranged from 28 to $57 \mathrm{mg} / \mathrm{kg}$; mercury ranged from 0.05 to $0.20 \mathrm{mg} / \mathrm{kg}$; and zinc ranged from 92 to $208 \mathrm{mg} / \mathrm{kg}$. Trace-element concentrations decreased near the mouth of Bayou Lafourche, possibly due to the effects of salinity. Trace elements tend to precipitate out of solution or suspension at freshwater-saltwater interfaces (Forstner and Wittmann, 1981, p. 191-194). Overall, the trace-element data indicate that Bayou Lafourche is adversely affected by low-level contamination upstream from the saltwater-freshwater interface generally located near Golden Meadow. 


\section{Nutrients}

Concentrations of dissolved nitrate in the Mississippi River at Baton Rouge are higher than other Louisiana streams, averaging $1.4 \mathrm{mg} / \mathrm{L}$ (milligrams per liter) during 1991-95 (fig. 17). This is far below the USEPA MCL of $10 \mathrm{mg} / \mathrm{L}$ for drinking water. This concentration can contribute to excessive plant growth, both of submerged aquatic vegetation and phytoplankton. In the spring through the fall of 1996, Bayou Lafourche had a large amount of submerged aquatic vegetation (SAV). The submerged aquatics caused water velocities to decrease, bayou stage to increase, and deposition of suspended sediments to increase. In June and October 1996, CWPPRA authorized the USGS to conduct water-quality surveys of Bayou Lafourche. The results of nutrient analyses indicated that the large amounts of SAV present in the bayou from Thibodaux to Lockport stripped the available dissolved nitrates from the water column (fig. 18), outcompeting the phytoplankton and causing the water column to be extremely clear and colorless. Late in 1996, the Lafourche Parish Water District began a program to remove excess SAV from the bayou. The program was successful in clearing the channel of most of SAV, as of the summer of 1997 (Kirk Cheramie, personal commun., 1997). However, this can increase the possibility of algal blooms. Considering the high nitrate levels in Mississippi River source water, algal blooms and the associated hypoxic events and fish kills are more likely to occur after the competing SAV are removed. Although not a threat to human health, taste and odor problems in drinking water often are associated with algal blooms.

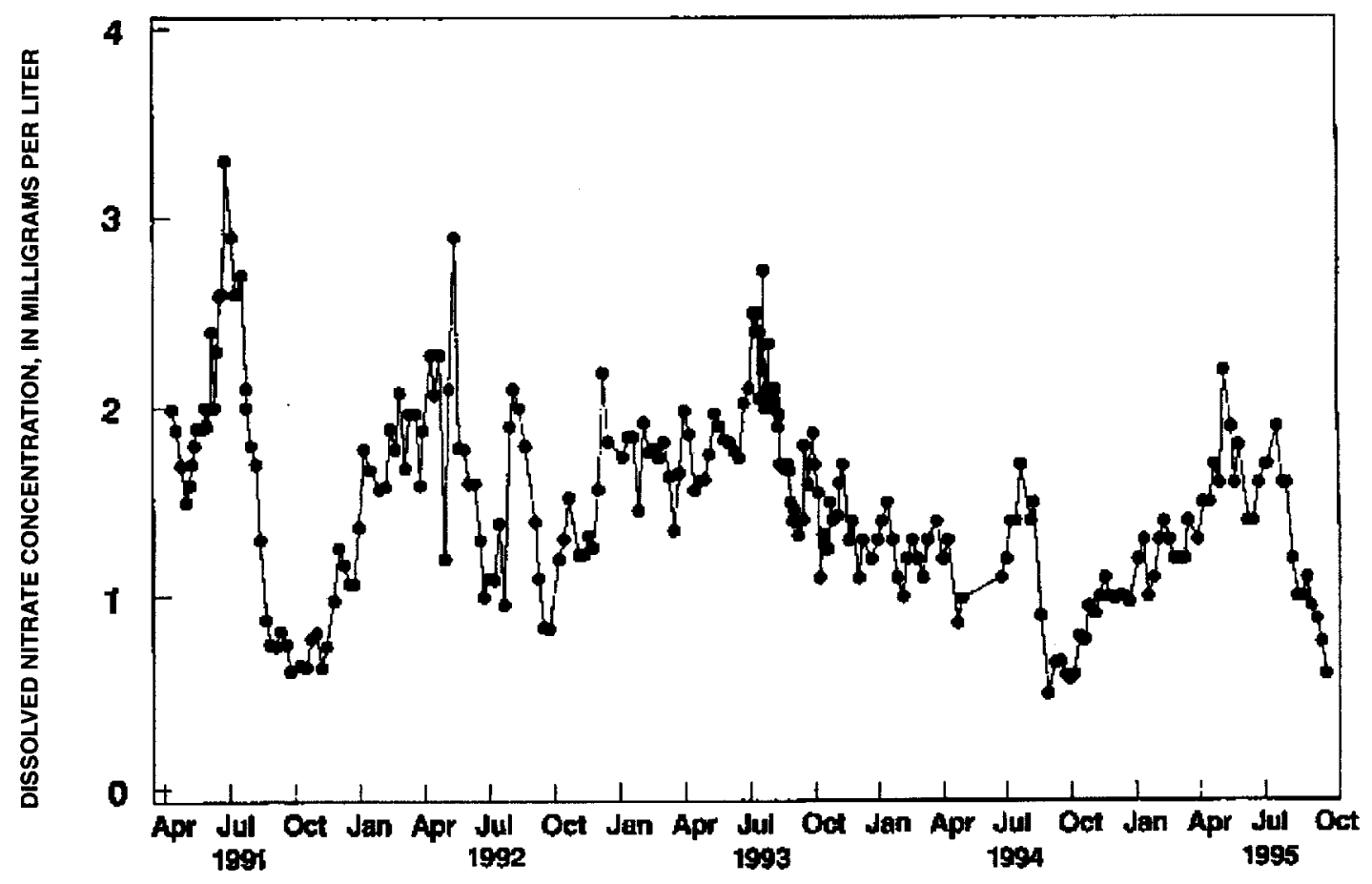

Figure 17. Dissolved nitrate concentration in the Mississippi River at Baton Rouge, Louisiana, 1991-95. 


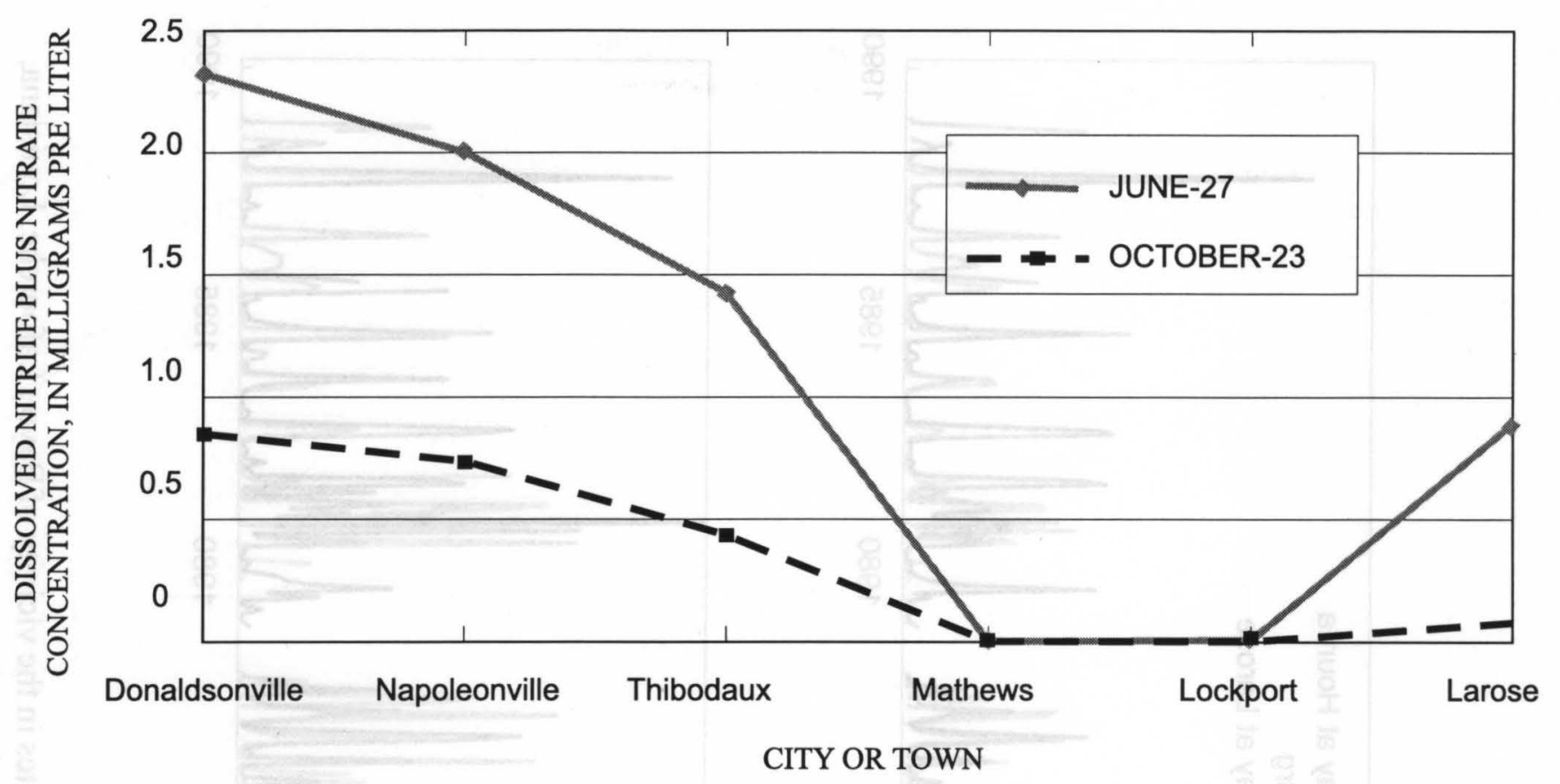

Figure 18. Dissolved nitrite plus nitrate concentration, Bayou Lafourche, Louisiana, June 27 and October 23, 1996.

\section{Salinity}

Long-term records of salinities at three stations near Bayou Lafourche and the GIWW, sampled by the COE, show no pronounced trends (fig. 19). In the GIWW at Houma, salinities remained low until 1961, when the GIWW was connected to coastal waters through the Houma Navigation Canal (HNC) (fig. 15). Peak mean monthly salinities occurred at all three sites at the same time (fig. 19A). Peaks were generally higher in the GIWW at Larose, compared to the GIWW at Houma and Bayou Terrebonne at Bourg, Louisiana. Maximum monthly salinities were generally higher at Bayou Terrebonne than at the other two sites (fig. 19B). The same data were subjected to the Kendall's Tau test (Swenson and Swarzenski, 1995) for trends and magnitude (tables 6 and 7). The GIWW at Larose apparently showed slightly decreasing salinities over time, whereas at Bayou Terrebonne, the trend was increased salinities. The periods of record were not the same, and the early period of salinity data at Houma may have been skewed because the HNC had not yet opened.

Encroaching saltwater is a threat to drinking water in the study area, as indicated in table 8 . At the intakes for the Terrebonne Waterworks District 1 at Houma, just east of the HNC, chloride concentrations exceeded the secondary maximum contaminant level (SMCL) ${ }^{1}$ of $250 \mathrm{mg} / \mathrm{L}$ during parts of every year except 1993 . Prior to completion of the HNC (1961), this was unusual (see fig. 19a). Frequently, chloride concentrations from the site on Bayou Lafourche at Valentine also exceeded standards (table 8). This site is located between Lockport and Larose. For the period 1990-96, chloride concentrations did not exceed USEPA standards in Bayou Lafourche at Mathews.

\footnotetext{
${ }^{1}$ Concentrations of chloride greater than $250 \mathrm{mg} / \mathrm{L}$ exceed the secondary maximum contaminant level (SMCL) for drinking water (U.S. Environmental Protection Agency, 1977, 1992). SMCL's are established for contaminants that can adversely affect the aesthetic quality of drinking water. At high concentrations or values, health implications as well as aesthetic degradation may also exist. SMCL's are not federally enforceable, but are intended as guidelines for the states.
} 

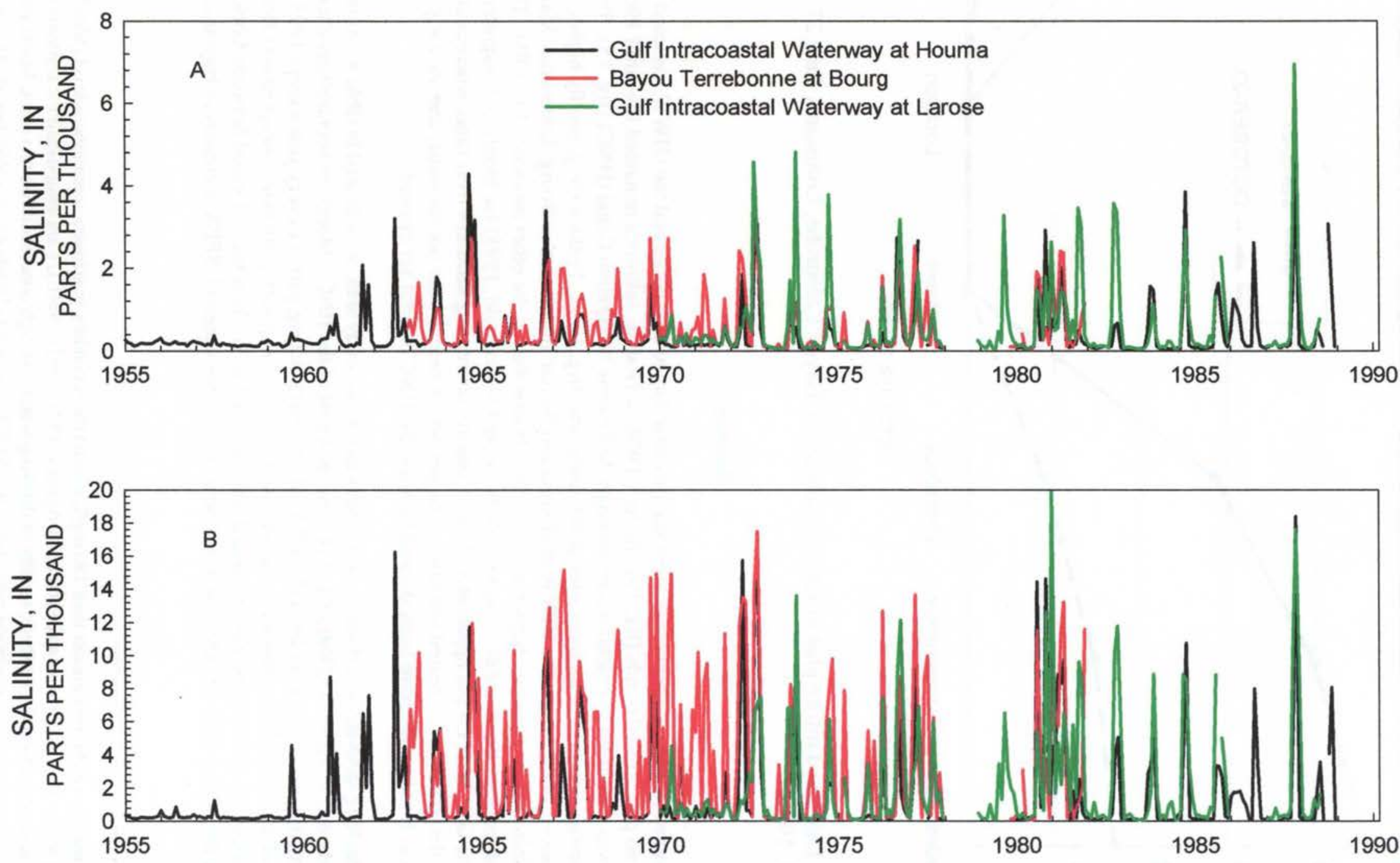

Figure 19. A, Mean monthly salinity, and B, maximum monthly salinity at selected sites in the vicinity of Bayou Lafourche, Louisiana, 1955-90 (Data from U.S. Army Corps of Engineers). 
Table 6. Daily mean and maximum salinity, and trends in magnitude for three sites in the Bayou Lafourche-Houma area, Louisiana

[Modified from: Swenson and Swarzenski, 1995; --, the value is less than the reporting level]

\begin{tabular}{ccccccc}
\hline & & & \multicolumn{2}{c}{$\begin{array}{c}\text { Salinity } \\
\text { (parts per thousand) }\end{array}$} & \multicolumn{2}{c}{$\begin{array}{c}\text { Trend } \\
\text { (parts per thousand) }\end{array}$} \\
\cline { 4 - 7 } $\begin{array}{c}\text { Station } \\
\text { number }\end{array}$ & Station name & $\begin{array}{c}\text { Period of } \\
\text { record }\end{array}$ & Mean & Maximum & Mean & $\begin{array}{c}\text { Maximum } \\
\text { monthly }\end{array}$ \\
\hline S76320 & $\begin{array}{l}\text { Gulf Intracoastal } \\
\text { Waterway at Houma }\end{array}$ & $1946-88$ & $0.4 \pm 1.06$ & 18.4 & -- & - \\
S76403 & $\begin{array}{l}\text { Bayou Terrebonne } \\
\text { at Bourg }\end{array}$ & $1962-82$ & $.6 \pm 1.59$ & 17.5 & +.041 & +.115 \\
\cline { 4 - 7 } S82203 & $\begin{array}{l}\text { Gulf Intracoastal } \\
\text { Waterway at Larose }\end{array}$ & $1970-88$ & $.6 \pm 1.48$ & 19.8 & -.034 & -.120 \\
\hline
\end{tabular}

Table 7. Months of lowest and highest salinity for three sites in the Bayou Lafourche-Houma area, Louisiana

[Modified from: Swenson and Swarzenski, 1995]

\begin{tabular}{lccc}
\hline \multicolumn{1}{c}{ Station name } & $\begin{array}{c}\text { Period of } \\
\text { record }\end{array}$ & $\begin{array}{c}\text { Months of } \\
\text { lowest salinity }\end{array}$ & $\begin{array}{c}\text { Months of highest } \\
\text { salinity }\end{array}$ \\
\hline Gulf Intracoastal Waterway at Houma & $1946-88$ & June, July & $\begin{array}{c}\text { October, November, } \\
\text { April }\end{array}$ \\
$\begin{array}{l}\text { Bayou Terrebonne at Bourg } \\
\text { Gulf Intracoastal Waterway at Larose }\end{array}$ & $1962-82$ & July & April, October \\
& $1970-88$ & $\begin{array}{c}\text { February, } \\
\text { March, July }\end{array}$ & $\begin{array}{c}\text { April, October, } \\
\text { November }\end{array}$ \\
\hline
\end{tabular}

The source of saltwater encroaching on Bayou Lafourche is variable. Some saltwater originates in Bayou Lafourche south of the GIWW; at other times saltwater moving up the HNC has flowed east in the GIWW, north into Company Canal and southeast in Bayou Lafourche towards Larose. Hurricane flood gates at Bayou Lafourche south of the GIWW, and exceptionally low flows in Bayou Lafourche in 1996, due in large part to the invasion of SAV, have made generalizations about saltwater encroachment in the study area tenuous. A more rigorous analysis of historical data, supplemented with limited new sampling, would improve the current understanding of saltwater movement into the areas of concern on Bayou Lafourche and the entire Barataria-Terrebonne estuary. 
The USGS also has compiled salinity data at 12 sites in the Barataria-Terrebonne estuary; the data were collected by DHH as part of their shellfish monitoring program. Analysis of information in the Barataria-Terrebonne National Estuary Program Status and Trends publication number 20 (Reed, 1995) on hydrologic modification indicates no general coastwide trends of increase or decrease for salinity. However, there was a visible trend in an increase of "spikiness" after about 1960-62 (Reed, 1995, p. 189). The term "spikiness" refers to pulses in salinity which tend to be a factor of 2 or 3 greater than the baseline salinity. This is especially noticeable at the GIWW at Houma, Lafitte, and Barataria. Possibly this is a result of the dredging of the Barataria Waterway and the HNC (Reed, 1995, p. 189).

Table 8. Numbers of days per year exceeding chloride concentrations of 250 milligrams per liter at three sites in the Bayou Lafourche area, Louisiana

[Sources of data: Nicolaus Paper, Inc. for Valentine site; Terrebonne Waterworks District 1 for Gulf Intracoastal Waterway site; Lafourche Waterworks District 1 for Mathews site]

\begin{tabular}{|c|c|c|c|}
\hline Year & $\begin{array}{l}\text { Bayou Lafourche at } \\
\text { Valentine }\end{array}$ & $\begin{array}{c}\text { Gulf Intracoastal } \\
\text { Waterway east of Houma } \\
\text { Navigation Canal at } \\
\text { Houma }\end{array}$ & $\begin{array}{c}\text { Bayou Lafourche at } \\
\text { Mathews }\end{array}$ \\
\hline 1972 & 3 & 39 & Data not available \\
\hline 1973 & 0 & 15 & \\
\hline 1974 & 0 & 30 & \\
\hline 1975 & 0 & 13 & \\
\hline 1976 & 3 & 57 & \\
\hline 1977 & 0 & 43 & \\
\hline 1978 & 0 & 40 & \\
\hline 1979 & 0 & 20 & \\
\hline 1980 & 0 & 32 & \\
\hline 1981 & 5 & 78 & \\
\hline 1982 & 4 & 28 & \\
\hline 1983 & 0 & 52 & \\
\hline 1984 & 34 & 38 & \\
\hline 1985 & 6 & 87 & \\
\hline 1986 & 0 & 45 & \\
\hline 1987 & 28 & 49 & \\
\hline 1988 & 18 & 83 & \\
\hline 1989 & 3 & 5 & \\
\hline 1990 & 0 & 5 & 0 \\
\hline 1991 & 12 & 40 & 0 \\
\hline 1992 & 0 & 31 & 0 \\
\hline 1993 & 0 & 0 & 0 \\
\hline 1994 & 6 & 29 & 0 \\
\hline 1995 & 2 & 29 & 0 \\
\hline
\end{tabular}




\section{SUMMARY AND CONCLUSIONS}

Approximately $170 \mathrm{Mgal} / \mathrm{d}$ (million gallons per day) of ground- and surface-water was withdrawn from the Barataria-Terrebonne Basins in 1995. Of this amount, surface water accounted for 64 percent $(110 \mathrm{Mgal} / \mathrm{d})$ of the total withdrawal rates in the basins. The largest surface-water withdrawal rates were from Bayou Lafourche (40 Mgal/d), Bayou Boeuf (14 Mgal/d), and the Gulf Intracoastal Waterway (4.2 $\mathrm{Mgal} / \mathrm{d}$ ). The largest ground-water withdrawal rates were from the Mississippi River alluvial aquifer (29 $\mathrm{Mgal} / \mathrm{d}$ ), the Gonzales-New Orleans aquifer (9.5 Mgal/d), and the Norco aquifer (3.6 Mgal/d).

The amounts of water withdrawn in the basins in 1995 differed by category of use. Public water suppliers within the basins withdrew $41 \mathrm{Mgal} / \mathrm{d}$ of water. The five largest public water suppliers in the basins withdrew $30 \mathrm{Mgal} / \mathrm{d}$ of surface water; Terrebonne Waterworks District 1 withdrew the largest amount, almost $15 \mathrm{Mgal} / \mathrm{d}$. Industrial facilities withdrew $88 \mathrm{Mgal} / \mathrm{d}$, fossil-fuel plants withdrew $4.7 \mathrm{Mgal} / \mathrm{d}$, and commercial facilities withdrew $0.67 \mathrm{Mgal} / \mathrm{d}$. Aggregate water-withdrawal rates, compiled by parish for aquaculture $(37 \mathrm{Mgal} / \mathrm{d})$, livestock $(0.56 \mathrm{Mgal} / \mathrm{d})$, rural domestic $(0.44 \mathrm{Mgal} / \mathrm{d})$, and irrigation uses $(0.54$ $\mathrm{Mgal} / \mathrm{d}$ ), totaled about $38 \mathrm{Mgal} / \mathrm{d}$ in the basins. Ninety-five percent of aquaculture withdrawal rates, primarily for crawfish and alligator farming, were from surface-water sources.

Total water-withdrawal rates increased 221 percent from 1960-95. Surface-water withdrawal rates increased by 310 percent, and ground-water withdrawal rates increased by 133 percent. The projection for the total water-withdrawal rates in 2020 is $220 \mathrm{Mgal} / \mathrm{d}$, an increase of 30 percent from 1995. Surface-water withdrawal rates would account for 59 percent of the total, or $130 \mathrm{Mgal} / \mathrm{d}$. Surface-water withdrawal rates are projected to increase by 20 percent from 1995 to 2020 . The decrease in surface-water withdrawal rates in 1985 is due in part to a decrease in industrial withdrawal rates for that year. The increase in withdrawal rates in 1990 and subsequent decrease in 1995 are mainly due to the different methods in calculating the amount of water used in crawfish farming. Past trends of ground-and surface-water use show an uneven pattern with a net increase over time. Taking this into account, the 30 percent increase in total withdrawal rates from 1995 to 2020 within the basins would probably follow an uneven pattern.

Bayou Lafourche is being evaluated as a means of conveying additional freshwater from the Mississippi River to coastal Louisiana marshes. Discharge measurements made in 1995-97 have shown that the Gulf Intracoastal Waterway has carried up to 4,000 cubic feet per second of water eastward across Bayou Lafourche.

Analysis of water quality of the lower Mississippi River indicates that the main threats to surfacewater resources are from the herbicide, atrazine, and from excessive nutrients. An understanding of Mississippi River water quality is important because Bayou Lafourche receives almost all of its discharge from the river. Atrazine concentrations in the Mississippi River at Baton Rouge exceed the U.S.

Environmental Protection Agency maximum contaminant level of 3.0 micrograms per liter during the late spring and early summer. However, as the maximum contaminant level is based on yearly average samples, the concentrations detected during the annual spring flush do not represent an exceedence of the criterion. Although trace elements should not pose any serious ecological problems in the lower Mississippi River, trace-metals in bottom material collected from Bayou Lafourche indicate that the reach of Bayou Lafourche from Donaldsonville to Golden Meadow is adversely affected by low-level contamination. Dissolved nitrate had a mean concentration of 1.4 milligrams per liter from 1991-95 in the Mississippi River near Bayou Lafourche. 
Long-term salinity records near Bayou Lafourche indicate no pronounced trends. In the Gulf Intracoastal Waterway at Houma, salinities remained low until 1961, when the Gulf Intracoastal Waterway was connected to the Gulf of Mexico by the Houma Navigation Canal. The source of saltwater encroaching on Bayou Lafourche is variable. Some saltwater has entered Bayou Lafourche south of the Gulf Intracoastal Waterway; at other times saltwater has moved up the Houma Navigation Canal and has flowed east in the Gulf Intracoastal Waterway, north into Company Canal and southeast in Bayou Lafourche towards Larose, Louisiana.

\section{SELECTED REFERENCES}

Bieber, P.P., and Forbes, M.J., Jr., 1966, Pumpage of water in Louisiana, 1965: Department of Conservation, Louisiana Geological Survey, and Louisiana Department of Public Works Water Resources Pamphlet no. 20, 8 p.

Britton, L.J., and Greeson, P.E., eds., 1988, Methods for the collection and analysis of aquatic biological and microbiological samples: U.S. Geological Survey Open-File Report 88-0190, 685 p.

Cardwell, G.T., and Walter, W.H., 1979, Pumpage of water in Louisiana, 1975: Louisiana Department of Transportation and Development, Office of Public Works Water Resources Special Report no. 2, 15 p.

Demas, C.R., and Curwick, P.B., 1987, Suspended-sediment, bottom-material, and associated chemical data from the lower Mississippi River, Louisiana: Louisiana Department of Transportation and Development Water Resources Basic Records Report no. 14, 117 p.

Dial, D.C., 1970, Pumpage of water in Louisiana, 1970: Department of Conservation, Louisiana Geological Survey, and Louisiana Department of Public Works Water Resources Pamphlet no. 26, 10 p.

Fishman, M.J., and Friedman, L.C., eds., 1989, Methods for the determination of inorganic substances in water and fluvial sediments: U.S. Geological Survey Techniques of Water-Resources Investigations, book 5, chap. A1, $545 \mathrm{p}$.

Forstner, U., and Wittmann, G.T.W., 1981, Metal pollution in the aquatic environment (2d revised ed.): New York, Springer-Verlag, $486 \mathrm{p}$.

Garrison, C.R., Goree, B.B., Lovelace, W.M., Montgomery, P.A., and Resweber, J.C., 1997, Water resources data-Louisiana, water year 1996: U.S. Geological Survey Water-Data Report LA-97-1, 495 p.

Goolsby, D.A., Thurman, E.M., Kolpin, D.W., and Battaglin, W.A., 1995, Occurrence of herbicides and metabolites in surface water, and rainwater in the Midwestern United States: Proceedings of the American Water Works Association 1995 Annual Conference, June 18-22, 1995, Anaheim, California, p. 583-591.

Kinne, A.O., 1971, Salinity in Marine Ecology: New York, Wiley Science, vol. 1, part 1.

Louisiana Department of Environmental Quality, 1996, Water quality inventory 1996 in State of Louisiana water quality management plan, v. 5, pt. B: Baton Rouge, Louisiana, Louisiana Department of Environmental Quality, Office of Water Resources, Water Quality Management Division, 314 p.

Louisiana State University Department of Sociology and the Louisiana Population Data Center for the Louisiana Division of Administration, "Post-Censal Population Projections to 2010 of Louisiana Parishes," 1994, accessed August 1, 1996, at URL http://www.state.la.us/state/census/parish/index.htm 
Lovelace, J.K., 1991, Water use in Louisiana, 1990: Louisiana Department of Transportation and Development Water Resources Special Report no. 6, 131 p.

---1994, Water requirements for crawfish farming at selected sites in south-central Louisiana, 1992-94: Louisiana Department of Transportation and Development Water Resources Special Report no. 8, 12 p.

Lovelace, J.K., and Johnson, P.M., 1996, Water use in Louisiana, 1995: Louisiana Department of Transportation and Development, Water Resources Special Report no. 11, 127 p.

Lovelace, J.K., and Lovelace, W.M., 1995, Hydrogeologic unit nomenclature and computer codes for aquifers and confining units in Louisiana: Louisiana Department of Transportation and Development Water Resources Special Report no. 9, 12 p.

Lurry, D.L., 1987, Pumpage of water in Louisiana, 1985: Louisiana Department of Transportation and Development, Office of Public Works Water Resources Special Report no. 4, 14 p.

McGee, B.D., and Demcheck, D.K., 1995, Occurrence and estimation of trace elements in bottom material for selected streams in coastal Louisiana, 1991-92: Louisiana Department of Transportation and Development Water Resources Technical Report no. 57, 49 p.

Moore, D.M., and Rivers, R.D, 1995, The estuary compact: A public-private promise to work together to save the Barataria and Terrebonne Basins: Thibodaux, La., Barataria-Terrebonne National Estuary Program, 40 p.

Reed, D.J., ed., 1995, Status and trends of hydrologic modification, reduction in sediment availability, and habitat loss/modification in the Barataria-Terrebonne estuarine system: Thibodaux, Louisiana, Barataria-Terrebonne National Estuary Program, BTNEP Publication no. 20, 338 p. plus appendices.

Shelton, L.R., 1994, Field guide for collecting and processing stream-water samples for the National Water-Quality Assessment Program: U.S. Geological Survey Open-File Report 94-455, 42 p.

Snider, J.L., and Forbes, M.J., Jr., 1961, Pumpage of water in Louisiana, 1960: Louisiana Department of Public Works, Department of Conservation, and Louisiana Geological Survey, $6 \mathrm{p}$.

Spearing, Darwin, 1995, Roadside geology of Louisiana: Missoula, Montana, Mountain Press Publishing Company, $225 \mathrm{p}$.

Swenson, E.M., and Swarzenski, C.M., 1995, Water levels and salinity in the Barataria-Terrebonne estuarine system, Part 6 of Reed, Denise, ed., Status and historical trends of hydrologic modification, reduction in sediment availability, and habitat loss/modification in the Barataria and Terrebonne estuarine system, Thibodaux, La., Barataria-Terrebonne National Estuary Program, BTNEP Publication no. 20, 338 p. plus appendices.

Urban Information Center, “Missouri State Census Data Center Population Trends Report, 1960-1995," University of Missouri-St. Louis, March 1996, accessed August 1, 1996, at URL http://www.oseda.missouri.edu/cgi-bin/browse?/mscdc/reports/poptrends/la

Walter, W.H., 1982, Pumpage of water in Louisiana, 1980: Louisiana Department of Transportation and Development, Office of Public Works Water Resources Special Report no. 3, 15 p.

Wells, F.C., 1980, Hydrology and water quality of the lower Mississippi River: Louisiana Department of Transportation and Development, Office of Public Works Water Resources Technical Report no. 21, 83 p.

Wiseman, W.J., Jr., and Swenson, E.M., 1988, Long-term trends in Louisiana estuaries, Chapter 6 of Turner, R.E., and Cahoon, D.R., eds., Causes of wetlands loss in the Coastal Central Gulf of Mexico, Vol. II: Technical Narrative. Minerals Management Service, New Orleans, Louisiana, OCS/MMS 87-0119, 400 p. 



\section{Appendix A:}

Aggregate Ground- and Surface-Water Withdrawal Rates And Usage in the Barataria-Terrebonne Basins, Louisiana, 1995 
Appendix A: Aggregate ground-and surface-water withdrawal rates and usage in the Barataria-Terrebonne Basins, Louisiana, 1995

[Mgal/d, million gallons per day; DO, domestic; LV, livestock; AQ, aquaculture; IR, irrigation; GW, ground water; SW, surface water, Misc, miscellaneous]

\begin{tabular}{|c|c|c|c|c|c|c|}
\hline \multirow{2}{*}{$\begin{array}{c}\text { Facility } \\
\text { identifi- } \\
\text { cation } \\
\text { number }\end{array}$} & \multirow[b]{2}{*}{ Parish or facility } & \multirow[b]{2}{*}{ Use } & \multirow[b]{2}{*}{ Type } & \multicolumn{2}{|c|}{ Source } & \multirow{2}{*}{$\begin{array}{l}\text { Withdrawal } \\
\text { rate }(\mathrm{Mgal} / \mathrm{d})\end{array}$} \\
\hline & & & & Surface water & Aquifer ${ }^{1}$ & \\
\hline \multirow[t]{2}{*}{379} & Ascension Parish & DO & GW & & 112MRVA & 0.054 \\
\hline & & DO & GW & & 112 NORC & 0.027 \\
\hline \multirow[t]{2}{*}{1157} & Ascension Parish & LV & GW & & 112MRVA & 0.013 \\
\hline & & $\mathrm{LV}$ & GW & & 112NORC & 0.003 \\
\hline 1349 & Ascension Parish & $\mathrm{AQ}$ & GW & & 112MRVA & 0.011 \\
\hline 391 & Assumption Parish & DO & GW & & 112MRVA & 0.029 \\
\hline 1158 & Assumption Parish & LV & SW & Misc streams & & 0.004 \\
\hline \multirow[t]{3}{*}{1350} & Assumption Parish & $\mathrm{AQ}$ & SW & Misc streams & & 0.644 \\
\hline & & $\mathrm{AQ}$ & SW & Lake Verret & & 0.625 \\
\hline & & $\mathrm{AQ}$ & SW & Bayou Lafourche & & 0.625 \\
\hline 597 & Iberville Parish & DO & GW & & 112MRVA & 0.109 \\
\hline \multirow[t]{2}{*}{1172} & Iberville Parish & LV & SW & Misc streams & & 0.007 \\
\hline & & LV & GW & & 112MRVA & 0.024 \\
\hline \multirow[t]{2}{*}{1364} & Iberville Parish & $\mathrm{AQ}$ & GW & & 112MRVA & 0.189 \\
\hline & & $A Q$ & SW & Misc streams & & 7.870 \\
\hline 1174 & Jefferson Parish & $\mathrm{LV}$ & SW & Misc streams & & 0.003 \\
\hline 1366 & Jefferson Parish & $A Q$ & SW & Misc streams & & 0.089 \\
\hline 311 & Lafourche Parish & DO & GW & & 112MRVA & 0.021 \\
\hline \multirow[t]{3}{*}{1148} & Lafourche Parish & LV & SW & Misc streams & & 0.048 \\
\hline & & LV & GW & & 112MRVA & 0.058 \\
\hline & & LV & GW & & 112MRVA & 0.135 \\
\hline \multirow[t]{5}{*}{1340} & Lafourche Parish & $\mathrm{AQ}$ & GW & & 112MRVA & 0.025 \\
\hline & & $\mathrm{AQ}$ & SW & Misc streams & & 3.803 \\
\hline & & AQ & SW & Bayou Lafourche & & 3.691 \\
\hline & & $\mathrm{AQ}$ & SW & $\begin{array}{l}\text { Intracoastal } \\
\text { Waterway }\end{array}$ & & 3.691 \\
\hline & & $\mathrm{AQ}$ & GW & & 112MRVA & 0.087 \\
\hline 1183 & Plaquemines Parish & $\mathrm{LV}$ & SW & Misc streams & & 0.075 \\
\hline 1375 & Plaquemines Parish & AQ & SW & Misc streams & & 0.802 \\
\hline \multirow[t]{8}{*}{844} & Pointe Coupee Parish & DO & GW & & 112MRVA & 0.007 \\
\hline & & DO & GW & & $12101 \mathrm{FP}$ & 0.003 \\
\hline & & DO & GW & & $12102 \mathrm{FP}$ & 0.017 \\
\hline & & DO & GW & & 12110BR & 0.003 \\
\hline & & DO & GW & & $12112 \mathrm{BR}$ & 0.010 \\
\hline & & DO & GW & & 12117BR & 0.014 \\
\hline & & DO & GW & & $12203 \mathrm{FP}$ & 0.010 \\
\hline & & DO & GW & & $12220 \mathrm{BR}$ & 0.048 \\
\hline
\end{tabular}


Appendix A: Aggregate ground-and surface-water withdrawal rates and usage in the Barataria-Terrebonne Basins, Louisiana, 1995-Continued

\begin{tabular}{|c|c|c|c|c|c|c|}
\hline \multirow{2}{*}{$\begin{array}{l}\text { Facility } \\
\text { identifi- } \\
\text { cation } \\
\text { number }\end{array}$} & \multirow[b]{2}{*}{ Parish or facility } & \multirow[b]{2}{*}{ Use } & \multirow[b]{2}{*}{ Type } & \multicolumn{2}{|c|}{ Source } & \multirow{2}{*}{$\begin{array}{l}\text { Withdrawal } \\
\text { rate }(\mathrm{Mgal} / \mathrm{d})\end{array}$} \\
\hline & & & & Surface water & Aquifer ${ }^{1}$ & \\
\hline & & DO & GW & & 12224BR & 0.021 \\
\hline & & DO & GW & & 12228BR & 0.010 \\
\hline \multirow[t]{4}{*}{1184} & Pointe Coupee Parish & LV & GW & & $12102 \mathrm{FP}$ & 0.020 \\
\hline & & LV & GW & & 12112BR & 0.010 \\
\hline & & LV & GW & & 12224BR & 0.010 \\
\hline & & LV & GW & & 12228BR & 0.010 \\
\hline \multirow[t]{2}{*}{1312} & Pointe Coupee Parish & IR & GW & & 112MRVA & 0.490 \\
\hline & & IR & SW & Misc streams & & 0.049 \\
\hline \multirow[t]{5}{*}{1376} & Pointe Coupee Parish & $\mathrm{AQ}$ & GW & & $12102 \mathrm{FP}$ & 0.089 \\
\hline & & $\mathrm{AQ}$ & GW & & 12112BR & 0.045 \\
\hline & & $\mathrm{AQ}$ & GW & & 12224BR & 0.045 \\
\hline & & $\mathrm{AQ}$ & GW & & 12228BR & 0.045 \\
\hline & & $\mathrm{AQ}$ & SW & Misc streams & & 2.081 \\
\hline \multirow[t]{2}{*}{896} & St. Charles Parish & DO & GW & & 112GRMC & 0.005 \\
\hline & & DO & GW & & $112 \mathrm{NORC}$ & 0.005 \\
\hline \multirow[t]{4}{*}{1189} & St. Charles Parish & LV & SW & Misc streams & & 0.027 \\
\hline & & LV & GW & & 111PNBR & 0.003 \\
\hline & & LV & GW & & 112GRMC & 0.013 \\
\hline & & LV & GW & & 112 NORC & 0.007 \\
\hline 1381 & St. Charles Parish & $\mathrm{AQ}$ & SW & Misc streams & & 0.767 \\
\hline \multirow[t]{3}{*}{918} & St. James Parish & DO & GW & & 112GRMC & 0.002 \\
\hline & & DO & GW & & 112MRVA & 0.001 \\
\hline & & DO & GW & & 112 NORC & 0.002 \\
\hline \multirow[t]{2}{*}{1191} & St. James Parish & LV & GW & Misc streams & $112 \mathrm{GRMC}$ & 0.001 \\
\hline & & LV & GW & & 112MRVA & 0.001 \\
\hline \multirow[t]{3}{*}{1383} & St. James Parish & $\mathrm{AQ}$ & SW & Misc streams & & 8.021 \\
\hline & & $\mathrm{AQ}$ & GW & & 112GRMC & 0.002 \\
\hline & & $\mathrm{AQ}$ & GW & & 112MRVA & 0.001 \\
\hline 1384 & St. John Parish & $\mathrm{AQ}$ & SW & Misc streams & & 2.869 \\
\hline 314 & Terrebonne Parish & DO & GW & & 112MRVA & 0.008 \\
\hline \multirow[t]{2}{*}{1151} & Terrebonne Parish & LV & SW & Misc streams & & 0.006 \\
\hline & & LV & GW & & 112MRVA & 0.054 \\
\hline 1343 & Terrebonne Parish & $\mathrm{AQ}$ & SW & Misc streams & & 17.443 \\
\hline \multirow[t]{3}{*}{1069} & West Baton Rouge Parish & DO & GW & & 11204BR & 0.001 \\
\hline & & DO & GW & & 11206BR & 0.003 \\
\hline & & DO & GW & & 112MRVA & 0.005 \\
\hline
\end{tabular}


Appendix A: Aggregate ground-and surface-water withdrawal rates and usage in the Barataria-Terrebonne Basins, Louisiana, 1995-Continued

\begin{tabular}{ccccccc}
\hline \multirow{2}{*}{$\begin{array}{c}\text { Facility } \\
\text { identifi- } \\
\text { cation } \\
\text { number }\end{array}$} & Parish or facility & Use & Type & Surface water & Aquifer ${ }^{1}$ & Withdrawal \\
\cline { 6 - 6 } rate (Mgal/d)
\end{tabular}

${ }^{1}$ Aquifer: 112MRVA, Mississippi River alluvial; 112NORC, Norco; 111SLNO, Shallow aquifers of New Orleans area; 112GRMC, Gramercy; 12101FP, Zone 1 Florida Parishes and Pointe Coupee Parish; 12102FP, Zone 2 Florida Parishes and Pointe Coupee Parish; 12110BR, "1,000-foot" sand of Baton Rouge area; 12112BR, "1,200-foot" sand of Baton Rouge area; 12117BR, "1,700-foot" sand of Baton Rouge area; 12203FP, Zone 3 Florida Parishes and Pointe Coupee Parish; 12220BR, "2,000-foot" sand of Baton Rouge area; 12224BR, "2,400-foot" sand of Baton Rouge area; 12228BR, "2,800-foot" sand of Baton Rouge area; 111 PNBR, Point-bar deposits; 11204BR, "400-foot" sand of Baton Rouge area; 11206BR, "600-foot" sand of Baton Rouge area; 112SLBR, Shallow sands of Baton Rouge area; 12115BR, "1,500-foot" sand of Baton Rouge area; 12223BR, "2,000 and 2,400 foot sands of Baton Rouge area. 


\section{Appendix B:}

Surface-Water Withdrawal Sites along the Mississippi River adjacent to the Barataria-Terrebonne Basins, Louisiana 


\section{Appendix B: Surface-water withdrawal sites along the Mississippi River adjacent to the}

Barataria-Terrebonne Basins, Louisiana

[IN, industry; WS, public supply; PF, fossil fuel power; $\mathrm{CO}$, commercial; PN, nuclear power]

\begin{tabular}{|c|c|c|c|c|c|}
\hline $\begin{array}{l}\text { Facility } \\
\text { identifi- } \\
\text { cation } \\
\text { number } \\
\text { (pl. 1) }\end{array}$ & Facility & Use & Source & Latitude & Longitude \\
\hline \multicolumn{6}{|c|}{ ASCENSION PARISH } \\
\hline 371 & Industry & IN & Mississippi River & 301344 & 0910317 \\
\hline 375 & Industry & IN & Mississippi River & 301115 & 0910044 \\
\hline 372 & Industry & IN & Mississippi River & 300611 & 0905732 \\
\hline 373 & Industry & IN & Mississippi River & 300730 & 0905443 \\
\hline 362 & Industry & IN & Mississippi River & 300815 & 0905452 \\
\hline 1722 & Industry & IN & Mississippi River & $1_{301300}$ & ${ }^{1} 0910200$ \\
\hline 361 & Industry & IN & Mississippi River & 301039 & 0910023 \\
\hline 363 & Industry & IN & Mississippi River & 300551 & 0905716 \\
\hline \multicolumn{6}{|c|}{ JEFFERSON PARISH } \\
\hline 1774 & Industry & IN & Mississippi River & 295450 & 0900820 \\
\hline 634 & Gretna Waterworks & WS & Mississippi River & 295529 & 0900324 \\
\hline 632 & Jefferson Parish Waterworks District 1 & WS & Mississippi River & 295739 & 0900953 \\
\hline 633 & Jefferson Parish Waterworks District 2 & WS & Mississippi River & 295357 & 0900543 \\
\hline 617 & Fossil fuel power plant & $\mathrm{PF}$ & Mississippi River & $1_{295600}$ & ${ }^{1} 0900800$ \\
\hline 635 & Westwego water system & wS & Mississippi River & 295438 & 0900835 \\
\hline \multicolumn{6}{|c|}{ ORLEANS PARISH } \\
\hline $744 a$ & New Orleans Sewage \& Water-Carrollton & WS & Mississippi River & 295727 & 0900746 \\
\hline $744 b$ & New Orleans Sewage \& Water-Algiers & WS & Mississippi River & 295634 & 0900746 \\
\hline 986 & Fossil fuel power plant & $\mathrm{PF}$ & $\begin{array}{l}\text { Mississippi River } \\
\text { Gulf Outlet }\end{array}$ & $1_{300030}$ & ${ }^{1} 0895610$ \\
\hline 985 & Fossil fuel power plant & $\mathrm{PF}$ & $\begin{array}{l}\text { Inner Harbor } \\
\text { Navigation Canal }\end{array}$ & ${ }^{1} 300056$ & ${ }^{I_{0}} 0900130$ \\
\hline \multicolumn{6}{|c|}{ PLAQUEMINES PARISH } \\
\hline 803 & Industry & IN & Mississippi River & 295143 & 0895819 \\
\hline 807 & Industry & IN & Mississippi River & $1_{294200}$ & ${ }^{1} 0895900$ \\
\hline 808 & Industry & $\mathrm{IN}$ & Mississippi River & 294833 & 0900027 \\
\hline $802 \mathrm{a}$ & $\begin{array}{l}\text { Plaquemines Parish Waterworks - Belle } \\
\text { Chase }\end{array}$ & WS & Mississippi River & 294817 & 0901051 \\
\hline $802 b$ & Plaquemines Parish Waterworks - Buras & WS & Mississippi River & 292130 & 0892607 \\
\hline $802 \mathrm{c}$ & $\begin{array}{l}\text { Plaquemines Parish Waterworks - } \\
\text { Dalcour }\end{array}$ & WS & Mississippi River & 295143 & 0895519 \\
\hline $802 d$ & $\begin{array}{l}\text { Plaquemines Parish Waterworks - } \\
\text { Pointe-a-la-Hache }\end{array}$ & WS & Mississippi River & 293503 & 0894826 \\
\hline $802 \mathrm{e}$ & $\begin{array}{l}\text { Plaquemines Parish Waterworks - Port } \\
\text { Sulphur }\end{array}$ & WS & Mississippi River & ${ }^{1} 293500$ & $\mathrm{I}_{0894800}$ \\
\hline 804 & Industry & IN & Mississippi River & 291318 & 0892339 \\
\hline
\end{tabular}


Appendix B: Surface-water withdrawal sites along the Mississippi River adjacent to the Barataria-Terrebonne Basins, Louisiana-Continued

\begin{tabular}{|c|c|c|c|c|c|}
\hline $\begin{array}{l}\text { Facility } \\
\text { identifi- } \\
\text { cation } \\
\text { number } \\
\text { (pl. 1) }\end{array}$ & Facility & Use & Source & Latitude & Longitude \\
\hline \multicolumn{6}{|c|}{ ST. BERNARD PARISH } \\
\hline 1549 & Industry & $\mathrm{IN}$ & Mississippi River & 295554 & 0895849 \\
\hline 1847 & Industry & IN & Mississippi River & 295642 & 0900012 \\
\hline 999 & Industry & IN & Mississippi River & 295545 & 0895820 \\
\hline 878 & Industry & IN & Mississippi River & 295553 & 0895634 \\
\hline 880 & St. Bernard Water \& Sewage & WS & Mississippi River & 295545 & 0895734 \\
\hline \multicolumn{6}{|c|}{ ST. CHARLES PARISH } \\
\hline 889 & Commercial & $\mathrm{CO}$ & Mississippi River & ${ }^{1} 295700$ & ${ }^{\mathrm{I}_{09}} 01900$ \\
\hline 892 & Fossil fuel power plant & $\mathrm{PF}$ & Mississippi River & $1_{300100}$ & ${ }^{1} 0902700$ \\
\hline 893 & Fossil fuel power plant & $\mathrm{PF}$ & Mississippi River & 295950 & 0902750 \\
\hline 894 & Nuclear power plant & PN & Mississippi River & 295950 & 0902750 \\
\hline 888 & Industry & IN & Mississippi River & 295538 & 0902103 \\
\hline 895 & Industry & IN & Mississippi River & 295910 & 0902725 \\
\hline 890 & St. Charles Waterworks District 1 & WS & Mississippi River & 295900 & 0902330 \\
\hline 891 & St. Charles Waterworks District 2 & WS & Mississippi River & 295544 & 0902150 \\
\hline 885 & Industry & IN & Mississippi River & 295925 & 0902430 \\
\hline 887 & Industry & IN & Mississippi River & $1_{295900}$ & ${ }^{1} 0902700$ \\
\hline \multicolumn{6}{|c|}{ ST. JAMES PARISH } \\
\hline 909 & Industry & IN & Mississippi River & 300231 & 0904854 \\
\hline 911 & Industry & IN & Mississippi River & 300258 & 0904103 \\
\hline 912 & Industry & IN & Mississippi River & 300309 & 0903955 \\
\hline 916 & Lutcher water system & WS & Mississippi River & 300215 & 0904135 \\
\hline 917 & Gramercy water system & WS & Mississippi River & 300236 & 0904107 \\
\hline 1024 & Industry & IN & Mississippi River & $I_{300300}$ & ${ }^{1} 0904000$ \\
\hline 1550 & Industry & IN & Mississippi River & $1_{300200}$ & $\mathrm{l}_{0904800}$ \\
\hline 1551 & Industry & IN & Mississippi River & 300635 & 0905440 \\
\hline 907 & St. James Waterworks District 1 & WS & Mississippi River & 300021 & 0904749 \\
\hline 908 & St. James Waterworks District 2 & WS & Mississippi River & 300006 & 0904713 \\
\hline 2037 & Industry & IN & Mississippi River & 300531 & 0905450 \\
\hline \multicolumn{6}{|c|}{ ST. JOHN THE BAPTIST PARISH } \\
\hline 1853 & Commercial & $\mathrm{CO}$ & Mississippi River & 300318 & 0903408 \\
\hline 1553 & Industry & $\mathrm{IN}$ & Mississippi River & 300223 & 0902801 \\
\hline 921 & Industry & IN & Mississippi River & 300258 & 0903122 \\
\hline 922 & Industry & IN & Mississippi River & 300341 & 0903601 \\
\hline 923 & Industry & IN & Mississippi River & $1_{300300}$ & ${ }^{1} 0903800$ \\
\hline $989 \mathrm{a}$ & St. John Waterworks District 3 - Edgard & wS & Mississippi River & 300315 & 0903445 \\
\hline $989 \mathrm{~b}$ & St. John Waterworks District 3 - Lions & WS & Mississippi River & 300245 & 0903445 \\
\hline
\end{tabular}

$\mathrm{I}_{\text {Estimated latitude and longitude }}$ 